\title{
Uniaxially Stressed Ge:Ga and Ge:Be
}

\author{
OSCAR DANILO DUBON, Jr.
}

\author{
Engineering Division \\ Lawrence Berkeley Laboratory, 1 Cyclotron Rd. \\ Berkeley, CA 94720
}

and
Materials Science and Mineral Engineering Department
University of California
Berkeley, CA 94720

\section{M.S. Thesis}

December 1992

This work was supported by the National Physical Science Consortium, and by NASA Contract No. W-17,605 through the U.S. Department of Energy under Contract No. DE-AC03-76SF00098. 


\title{
Uniaxially stressed Ge:Ga and Ge:Be
}

by

Oscar Danilo Dubon, Jr.

\begin{abstract}
The application of a large uniaxial stress to p-type Ge single crystals changes the character of both the valence band and the energy levels associated with the acceptors. Changes include the splitting of the fourfold degeneracy of the valence band top and the reduction of the ionization energy of shallow acceptors. In order to study the effect of uniaxial stress on the transport properties of photoexcited holes, a variable temperature photo-Hall effect system was built in which stressed $\mathrm{Ge}: \mathrm{Ga}$ and $\mathrm{Ge}: \mathrm{Be}$ could be characterized. Results indicate that stress significantly increases the lifetime and the Hall mobility of photoexcited holes. These observations may help further the understanding of fundamental physical processes that affect the performance of stressed $\mathrm{Ge}$ photoconductors including the capture of holes by shallow acceptors.
\end{abstract}


1. Introduction

2. Semiconductor Basics 4

2.1 Band structure and effective mass theory 4

2.2 Monovalent impurities in germanium 9

$2.3 \mathrm{Be}$ : an example of a double acceptor in $\mathrm{Ge}$

2.4 Freeze-out statistics 13

2.5 Hole mobility in Ge 18

2.6 Non-radiative capture of holes by acceptors 22

2.7 Hall effect and photo-Hall effect 29

2.8 Effects of uniaxial stress 33

3. Experimental 36

3.1 Hall apparatus and measurement 36

3.2 Stressing coldfinger $\quad 40$

3.2.1 Temperature sensing resistor 41

3.2.2 The blackbody emitter and other considerations 43

3.2.3 Aspects of the stressing configuration 47

3.3 Materials selection and preparation 47

3.4 Sample mounting $\quad 50$

4. Results and discussion 52

4.1 Calibration results 52

4.1.1 Photon absorption rate at $77 \mathrm{~K}$ and $99 \mathrm{~K} \quad 52$

4.1.2 Stress calibration $\quad 55$ 
4.2 Experimental results in the Ge:Ga system

4.2.1 Freeze-out statistics where thermal generation dominates

4.2.2 Carrier lifetime 58

4.2.3 Hall mobilities

62

4.3 Experimental results in the Ge:Be system 65

4.3.1 Free carrier statistics and lifetimes in Ge:Be 65

4.3.2 Hall mobilities

73

4.4 Recombination coefficients for the Ge:Ga and

Ge:Be systems

77

5. Conclusion

82

6. References

84

7. Appendix A 


\section{Acknowledgments}

I would like to thank Professor Eugene Haller for giving me the opportunity to learn how to perform research. I am especially grateful to him for allowing me the freedom to struggle through my work and for giving me a push just when I have needed one. I would also like to thank Professor Paul Richards and Professor Eicke Weber for reviewing my thesis. Their thoughtful comments were very helpful.

I have had the fortune of working with an outstanding group of individuals. My deepest gratitude I extend to Jeff Beeman; his technical advice and, more importantly, his friendship have been invaluable to me. I am also very appreciative of the technical support of John Emes, R.C. Davis, Steve Rothway, and Margaret Ragsdale. Thanks to the student colleagues, past and present, for their words of encouragement and their contribution to my education through many fruitful discussions about science and life. I am particularly thankful to Dr. David Bliss for showing me how to use the Hall effect apparatus and keeping me company in the computer room as we wrote our theses.

Beyond individuals whom I have had the honor of meeting at Berkeley, I am very grateful to thank Professor Alfred Yue for introducing me to the field of materials science and Professor Nancy Haegel for being the consummate role model as both a scientist and a person. In addition, I would like to thank Tom Glasgow, MaryJo Meyer, and Bill Meyer at NASA Lewis Research Center for giving me the opportunity to work with them through summer research experiences. 
Finally, words cannot describe how grateful I am to my parents, Oscar and Sandra Dubon, for encouraging me to pursue my goals. Thanks to Leyla for proof reading important portions of this thesis.

I wish to acknowledge the support of the National Physical Science Consortium. 


\section{Introduction}

The photoconductive characteristics of semiconductors have led to the wide use of materials such as $\mathrm{Ge}, \mathrm{PbS}$, and $\mathrm{HgCdTe}$ for infrared (IR) radiation detection.1,2 Semiconductors are photoconductors because they can show an increase in electrical conductivity upon absorbing electromagnetic radiation. The absorption of photons can result in (a) the promotion of electrons from the valence band to the conduction band producing electron-hole pairs or (b) the excitation of electrons (holes) from an impurity level within the energy gap to the conduction (valence) band. Both processes increase the concentration of mobile carriers in a semiconductor thus increasing the material's conductivity as well. In process (a) the light interacts with the atoms of the semiconducting host; this is called intrinsic photoconductivity. Process (b) is termed extrinsic (or impurity) photoconductivity because the photoexcited charge carriers originate from impurity atoms.

Energy levels near the valence band edge of a semiconductor can be introduced by doping, the controlled addition of impurities. The presence of "shallow" levels makes the measurement of the photoconductivity behavior in doped semiconductors an effective method for long-wavelength photon detection.1,3 This has significant applications in several areas including IR astronomy and IR spectroscopy. Doped germanium photoconductors are well suited for detection of far IR radation (10-200 $\mu \mathrm{m})$. Dopants such as $\mathrm{Cu}, \mathrm{Zn}$, and $\mathrm{Be}$ produce an extrinsic photoconductivity behavior in Ge via hole excitation with photons of wavelengths in the $10-50 \mu \mathrm{m}$ range.4,5 Radiation between $50 \mu \mathrm{m}$ and 120 $\mu \mathrm{m}$ is detected with Ga- or B-doped Ge.3,6 In recent years the detection of 
$200 \mu \mathrm{m}$ photons has been accomplished with uniaxially stressed, Ga-doped Ge detectors. ${ }^{7}$ (Note: the energy, E, of a photon is obtained from its wavelength, $\lambda$, by the relation $E=h c / \lambda$, where $h$ is Planck's constant and $c$ is the speed of light in a vacuum.)

When a voltage bias is applied across a photoconductor, the increase in the material's conductivity upon the photoexcitation of charge carriers is measured as an increase in the electrical current flowing along the direction of the applied bias. The ability of the electrons (holes) to conduct depends on (a) how fast they can drift through the semiconductor and (b) how much time they spend in the conduction (valence) band before being captured by dopant impurities and/or recombining with carriers of opposite charge. The quantity describing the average carrier velocity per unit of applied electric field is the mobility $\mu$. The length of time carriers typically spend in the energy bands is described by the carrier lifetime. The effectiveness of a semiconductor as an IR detector is therefore determined by both the photon energy range over which the semiconductor is sensitive and the electrical characteristics of the detector.

The application of uniaxial stress to a Ga-doped Ge photoconductor not only changes the spectral range of detectable photons but also increases the photocurrent of the device. The former effect results from a reduction in the binding energy of the Ga level from which holes are photoexcited. An explanation for the increase in photocurrent is likely to be founded on the effect of uniaxial stress on both the mobility and lifetime of holes. The stress dependerve of mobility has been studied, 8.9 but the behavior of the carrier lifetime is not yet well understood.

The work presented in this thesis has two principal objectives: 1) to provide a method for using the phsto-Hall technique to study 
simultaneously the mobility and lifetime of uniaxially-stressed, semiconductor single crystals and 2) to report both quantitative as well as qualitative evidence for the change in carrier lifetime with stress in Gadoped and Be-doped $\mathrm{Ge}$. The chapter following this introduction summarizes the major concepts in semiconductor physics necessary as a background for this work. Chapter 3 describes the experimental requirements and the means by which these were met. Results are then presented and discussed in Chapter 4 . The thesis will end with some concluding comments. 


\section{Semiconductor Basics}

\subsection{Band structure and effective mass theory}

Energy bands in a crystal arise from the interaction of the valence electron wavefunctions of each atom with those of the other atoms. Each germanium atom possesses 4 valence electrons: two $4 \mathrm{~s}$ and two $4 p$ electrons. For the case of isolated atoms, the $4 s$ and $4 p$ energy levels are split, and all atoms can have this same electronic structure. But when the $\mathrm{Ge}$ atoms become covalently bonded, the wavefunctions of the valence electrons in the crystal overlap, and the Pauli exclusion principle forbids these bonding electrons from occupying the same energy state, (i.e., no two electrons can have the same quantum number). As a result each energy level splits, and essentially a continuum of allowed energies is formed constituting an energy band as depicted in Figure 2.1. In a covalently bonded crystal such as Ge, the $s$ and $p$ orbitals interact with each other creating s-p hybridization. This orbital mixing is reflected in the energy bands. The valence bands are composed of one s-and three p-states and can accommodate a total of four electrons per atom. Likewise, the conduction bands consist of one s-and three p-like bands although the mixing of the states does not result in the same band configuration as that of the valence bands. The minimum energy gap between the valence and conduction bands is called the bandgap and has a value of $0.67 \mathrm{eV}$ at room temperature for germanium (Fig. 2.2).

Because Ge has 4 valence electrons per atom, the valence bands are completely filled and the conduction bands are empty (at $\mathrm{T}=0 \mathrm{~K}$ ). (Note: any future reference to a valence or conduction band refers to those bands 


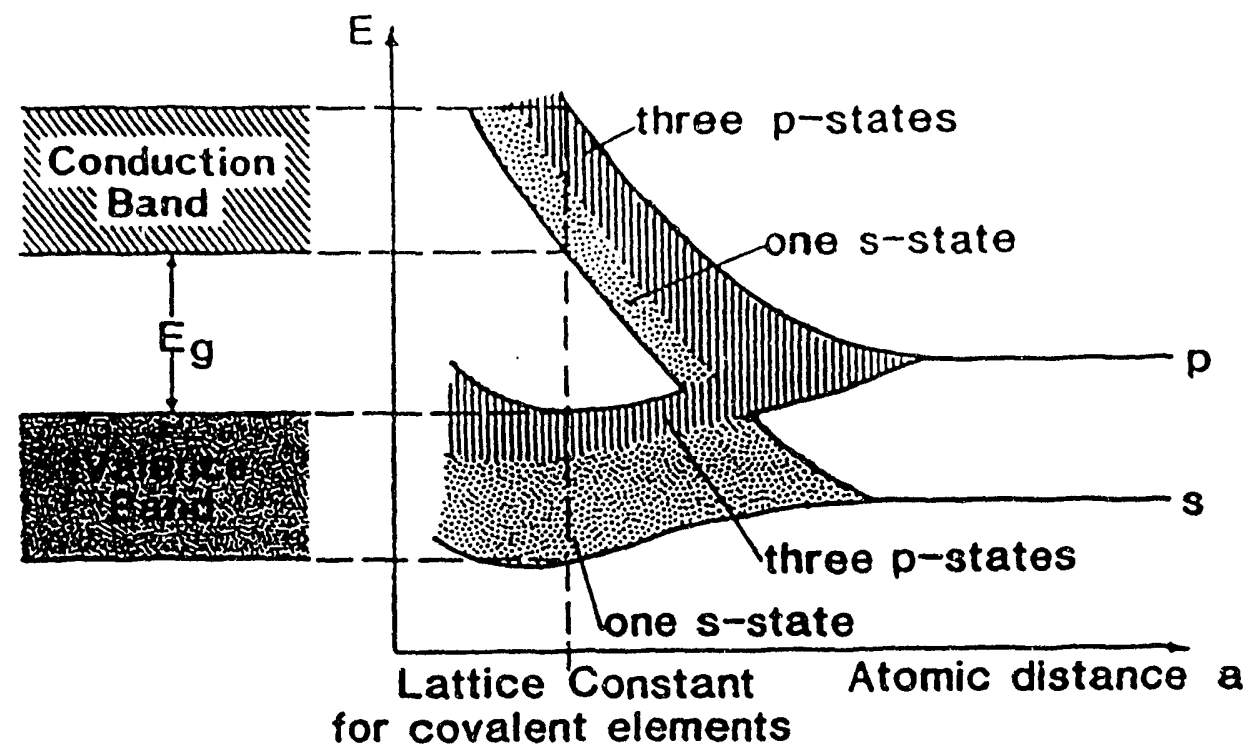

Figure 2.1. Sharp energy levels, widening into bands, and band overlapping with decreasing atomic distance for covalent elements such as Ge. 10
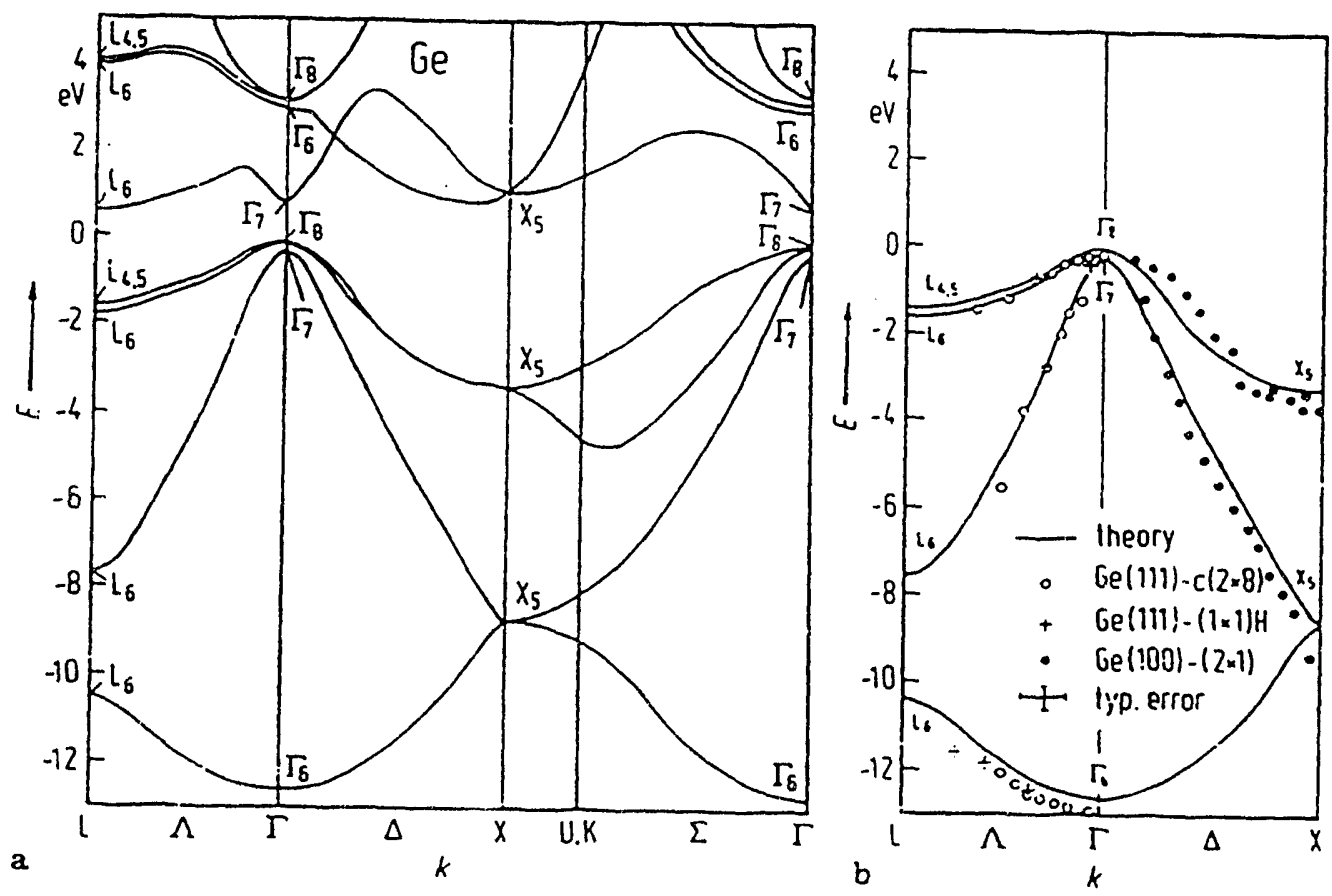

Figure 2.2. (a) Band structure of $\mathrm{Ge}$ obtained by a non-local pseudopotential calculation including spin orbit interaction,11 (b) comparison of the calculated valence bands with angular resolved photoemission data. ${ }^{12}$ 
whose maxima and minima form the bandgap.) So if an external field is applied, no electrical current will flow because all allowed energy states in the valence band are occupied, and the electrons will have no states into which to move. Instiators do not carry any appreciable current for the same reason. The magnitude of the bandgap determines whether a material is called insulator or semiconductor. If the gap is less than a few electron volts in magnitude, the material is a semiconductor; thus, Ge falls under this category.

In the case of semiconductors, the thermal energy at room temperature is sufficient to excitate a significant number of electrons from the valence band to the conduction band. Once the electrons are in the conduction band, there are many states available for them to occupy. In an electric field these excited charge carriers, electrons as well as holes, will be able to move through the crystal creating an electrical current.

In order to treat classically the motion of electrons and holes in the bands, effective mass theory (EMT) is applied. ${ }^{13}$ EMT brings together the wave-like and particle-like character of electrons and provides a means of using the classical equation of motion to current carrying electrons (holes) in crystals. It can be shown that a charge carrier travelling in an energy band behaves as if having an "effective" mass $\mathrm{m}^{*}$ given by

$$
\mathrm{m}^{*}=\left[\frac{1}{\hbar^{2}}\left(\frac{\mathrm{d}^{2} \mathrm{E}}{\mathrm{dk}^{2}}\right)\right]^{-1}
$$

This equation reveals that the effective mass is inversely proportional to the curvature of the energy band in momentum space-described in E vs. $k$ diagrams-which the electron (hole) occupies. Electrons conduct in bands 
with positive curvature while holes are the charge carriers in bands of negative curvature. In addition, the magnitude of the curvature of the band determines the magnitude of the mass; thus, a band of high curvature is populated with carriers of small effective mass typically leading to higher mobilities.

The top of the valence band in Ge (Fig. 2.2) and many other semicunductors is found at $\mathrm{k}=0$ and is fourfold degenerate. The bancis creating the fourfold degeneracy are, as mentioned before, constructed from $p$ orbitals with total angular momentum quantum number, $m_{j}$, of $3 / 2$. In addition, there exists a doubly degenerate band $\left(m_{j}=1 / 2\right)$ with its extremum also located at $k=0$. The spin-orbit interaction moves the top of this band, also called the split-off band, to higher hole energies. The splitoff band in $\mathrm{Ge}$ is separated from the valence band edge by $0.29 \mathrm{eV}$. This large split-off energy reduces the impact of this band on the transport and optical properties considered to negligible values. The structure of the valence band edge is very important because the current carrying holes populate this region of the band. The degeneracy of the valence band top must be taken into account in the formulation of the effective mass of holes. In fact, two separate effective masses are designated, one for each of the bands creating the fourfold degeneracy. The band with the larger curvature contributes a light-hole effective mass while the heavy holes are designated to the band of lower curvature.

Although the above expression of the effective mass holds for a onedimensional systems, it does not suffice to use this scalar formula to describe the three dimensional character of the band structure of a solid. The acceleration a that a charge carrier experiences under an applied force $F$ is 


$$
\mathbf{a}=\frac{\mathrm{d} \mathbf{v}}{\mathrm{dt}}=\frac{1}{\mathrm{~m}^{*}} \cdot \mathbf{F}
$$

where $1 / \mathrm{m}^{*}$ is a tensor relating a to $\mathbf{F}$, and each tensor component, $\mathrm{m}_{\mathrm{ij}}{ }^{-1}$, is given by

$$
\mathrm{m}_{\mathrm{ij}}^{-1}=\tilde{h}^{-2} \frac{\partial^{2} \mathrm{E}}{\partial \mathrm{k}_{\mathrm{i}} \partial \mathrm{k}_{\mathrm{j}}} .
$$

The interaction between the heavy and light hole bands in Ge produces allowed energy states near the valence band edge described by

$$
\mathrm{E}(\mathrm{k})=\mathrm{Ak}^{2} \pm\left[\mathrm{B}^{2} \mathrm{k}^{4}+\mathrm{C}^{2}\left(\mathrm{k}_{\mathrm{x}}^{2} \mathrm{k}_{\mathrm{y}}^{2}+\mathrm{k}_{\mathrm{y}}^{2} \mathrm{k}_{\mathrm{z}}^{2}+\mathrm{k}_{\mathrm{z}}^{2} \mathrm{k}_{\mathrm{x}}^{2}\right)\right]^{1 / 2}
$$

where $|k|^{2}=k_{x}{ }^{2}+k_{y}{ }^{2}+k_{z}{ }^{2}$. A, B, and $C$ are constants determining the magnitude of the components of the effective mass tensor. The plus sign corresponds to the heavy hole band and the minus to the light hole band. In the approximation of spherical constant energy surfaces, an average, scalar effective mass is obtained for heavy and light holes $\left(0.35 \mathrm{~m}_{e}\right.$ and $0.043 \mathrm{~m}_{e}$, respectively, where $\mathrm{m}_{\mathrm{e}}$ is the free electron mass) as in the onedimensional case since the principal axes are equivalent. Actual effective masses have been determined form cyclotron resonance experiments. ${ }^{14,15}$

The conduction band in $\mathrm{Ge}$ is characterized by a band minimum located at each of the eight equivalent $<111>$ directions at the Brillouin zone boundary. Each is characterized by ellipsoidal constant energy surfaces with the axis of revolution coincident with the associated $<111>$ direction. The allowed energy states near each minimum is given by

$$
\mathrm{E}(\mathrm{k}) \approx \mathrm{Ak}_{1}{ }^{2}+\mathrm{B}\left(\mathrm{k}_{2}{ }^{2}+\mathrm{k}_{3}{ }^{2}\right)
$$


where $k_{1}$ is oriented along the axis of revolution, and $k_{2}$ and $k_{3}$ are perpendicular to each other and to $\mathrm{k}_{1}$. Two effective masses are associated with such energy surfaces. The longitudinal effective mass $m_{\|}^{*}$ equal to $1.6 \mathrm{~m}_{e}$ defines the motion along $\mathrm{k}_{1}$ while the transverse mass $m_{\perp}{ }^{*}$ equal to $0.082 \mathrm{~m}_{\mathrm{e}}$ reflects the curvature of the surfaces in directions perpendicular to the axis of revolution.

\subsection{Monovalent impurity states in germanium}

When impurities are added to a semiconductor, energy states may be introduced in the bandgap of the material. The position of the energy level depends on the chemical nature of the impurity with which the state is associated. Group III and V impurities in Ge are monovalent dopants since they can provide one current carrier per atom leaving each impurity site singly charged. Group III elements are known as acceptors because they bind an extra electron typically leading to either the formation of a hole in the valence band or the compensation of a donor impurity. On other hand, column $\mathrm{V}$ elements provide an electron to the conduction band or an acceptor impurity, (i.e., compensation). These dopants are thus called donors. Although these elements are chemically distinct, many of their spectral features can be understood by the application of the concepts of dielectric screening and effective mass theory to the quantum mechanical model of the hydrogen atom.

The quantum mechanical solution of the hydrogen atom problem reveals that the electron is bound to the nucleus with a ground state energy of $13.6 \mathrm{eV}$. There exists a series of discrete bound excited states which is given by $13.6 \mathrm{Z}^{2} / \mathrm{n}^{2} \mathrm{eV}$ where $\mathrm{n}$ is the principal quantum number and $\mathrm{Z}$ is 
the number of unit charges in the nucleus (equal to 1 for hydrogen). The distance from the proton at which there is a maximum probability of finding the electron is given by the Bohr radius $a_{0}$ equal to $0.053 \mathrm{~nm}$. These results can be applied to group III and V impurities in $\mathrm{Ge}$ by multiplying (a) the permitivity in free space $\varepsilon_{0}$ with the dielectric constant of $\mathrm{Ge}$ and (b) the free electron mass with the electron or hole effective mass. These substitutions account for both the screening of the impurity nuclei caused by the electron cloud of the host atoms and the difference in the motion of electrons in energy bands versus free space. The two formulas for the hydrogen atom become

$$
\mathrm{E}=-\frac{13.6 \mathrm{~m}^{*}}{\varepsilon_{\mathrm{r}}^{2} \mathrm{~m}} \mathrm{eV} \quad \text { and } \quad \mathrm{r}^{\prime}=\frac{0.053 \mathrm{~m} \varepsilon_{\mathrm{r}}}{\mathrm{m}^{*}} \mathrm{~nm}
$$

where $\varepsilon_{\mathrm{r}}$ is the relative dielectric constant of the semiconductor and $r^{\prime}$ is the impurity Bohr radius. For Ge the ground state energy is $10 \mathrm{meV}$ and the Bohr radius is $8 \mathrm{~nm}$ for $\mathrm{m}^{*} \approx 0.2 \mathrm{~m}_{\mathrm{e}}$ and $\varepsilon_{\mathrm{r}}=16$. The orbiting electron (hole) is delocalized over many atomic spacings and only lightly bound to the impurity site as a result of the dielectric screening. Hence, these dopants have energy levels in the bandgap close to the respective band minima. They are also known as shallow, hydrogenic impurities.

The experimental evidence supports the general features of this model.16 The actual ionization energies of hydrogenic impurities vary from $10 \mathrm{meV}$ to $14 \mathrm{meV}$ depending on the element involved. Such deviations from the model reflect the validity of the assumptions made. In particular, the assumption that the core electrons screen the nucleus so as to make it seem like a single point charge is not completely accurate. When the electron (hole) is in the s-like ground state, it is more tightly bound to 
the nucleus due to the reduced screening by core electrons. A consequence of this is that the energy required to promote the orbiting charge from the ground state to an excited state is larger than expected. But the energy separation of the optically accessible, bound excited states follows exactly from the quantum mechanically more rigorous model (which takes into account the degeneracy of the valence band and the symmetry of the environment of the impurity). Because they are p-like in character with a node at $r=0$, the bound excited states do not interact strongly with the atom core.

\section{$2.3 \mathrm{Be}$ : an example of a double acceptor in $\mathrm{Ge}$}

Group III and V elements are not the only chemical species that can dope a Ge crystal. Column IIB and VIB elements can provide two holes and two electrons, respectively. Beryllium, which occupies a substitutional position in $\mathrm{Ge}$, can provide two holes for conduction and is therefore called a double acceptor. Although the hydrogen atom does not serve as an appropriate system from which to make quantum mechanical comparisons with $\mathrm{Be}$ in $\mathrm{Ge}$, the helium atom is a suitable analog for this double acceptor.

There are two major features which distinguish the He atom problem from that of the hydrogen atom. First, the nucleus has a charge of $+2 \mathrm{e}$; second, there are two orbiting electrons that interact with each other as well as with the nucleus. Electron-electron interaction must be taken into account in the calculation of the energy required for the atom to reach its first ionization state, (i.e., removing the first electron). The electrons feel both the electrostatic repulsion of one another and the Coulombic attraction 
of the nucleus resulting in a net field proportional to $Z^{\prime} e^{2} / r$ where $1<Z^{\prime}<2$ and $Z^{\prime}$ ' is the effective charge number of the atom core. A $Z$ ' between 1 and 2 results from the partial screening of the He nucleus by the electrons. Because both electrons are indistinguishable particles when in their ground state, they screen each other equally from the nucleus. Yet the ionization energy for the electron first removed will be smaller than that of the second electron. The removal of the first electron eliminates the additional screening from the electron-electron interaction, and the nucleus is fully exposed. So, the second electron experiences a nuclear charge of $+2 \mathrm{e}$ when the atom is in its first ionization state. This information can be used to understand the energy quantization of the bound excited states of the double acceptor $\mathrm{Be}$ in $\mathrm{Ge}$.

Each neutral beryllium atom in germanium binds two holes. The ground state is located $24.5 \mathrm{meV}$ from the valence band edge. Similar to the case of shallow hydrogenic impurities, holes may be optically excited from the ground state to bound states which are described by effective mass theory. The relative separation of these bound excited states corresponds closely to the spacing of the excited states of neutral single acceptors. In both cases the optically-accessible bound excited states are of p-like character, and the atom cores appear as a point charge $\left(Z^{\prime}=1\right)$ to holes located at any of these excited states. The neutral Be atom and the neutral, single acceptor are in these respects analogous to the neutral $\mathrm{He}$ and neutral $\mathrm{H}$ atoms, respectively.

Singly-ionized beryllium atoms in germanium each bind one hole. The ground state is located $58 \mathrm{meV}$ from the valence band edge. The spacing of the excited states associated with singly ionized Be atoms is four times that associated with neutral group III acceptor. Bound holes in the 
ionzed $\mathrm{Be}$ atoms are exposed to a core pointial having a $\mathrm{Z}^{\prime}$ equal to 2 in an analogous manner to the valence electron in the $\mathrm{He}^{+}$ion. The factor of four arises because the bound state energies are proportional to $\left(Z^{\prime}\right)^{2}$.

Although Be normally acts as a double acceptor, it can bind a third hole ${ }^{18}$ (designated as $\mathrm{Be}^{+}$) under certain conditions in a manner analogous to overcharged atomic hydrogen $\mathrm{H}^{-}$. The existence of $\mathrm{Be}^{+}$centers occurs at low temperatures and requires the presence of excess holes in the valence band. Like the Helium atom a Be impurity would only be able to accommodate two holes in its ground state if holes had spin $1 / 2$. But the fourfold degeneracy of the valence band top gives holes a pseudo spin of $3 / 2$, and this permits the ground state to be occupied with up to four holes without violation of the Pauli exclusion principle. Photoconductive response spectra performed at low temperature $18(<3 \mathrm{~K})$ show that the $\mathrm{Be}^{+}$ center has a binding energy of about $4 \mathrm{meV}$ and that it disappears upon the application of uniaxial compression. The stress reduces the degeneracy of the band edge from fourfold to two twofold split bands. The effect of stress on the valence band of $\mathrm{Ge}$ is discussed in Section 2.8

\subsection{Freeze-out statistics}

Under thermal equilibrium the free carrier density in the bands depends on both the likelihood of generating free carriers with the available thermal energy at a given temperature and the density of states in the bands to which these electrons and holes can be promoted. Therefore, the concentration of electrons in the conduction band, $n$, as a function of temperature is given by 


$$
\mathrm{n}=2\left(\frac{2 \pi m_{n}^{*} k_{B} T}{h^{2}}\right)^{3 / 2} \exp \left(\frac{E_{F}-E_{C}}{k_{B} T}\right)=N_{C} \exp \left(\frac{E_{F}-E_{C}}{k_{B} T}\right)
$$

The pre-exponential term $\mathrm{N}_{C}$ is called the effective density of conduction band states and may be interpreted as the density of states at the conduction band edge representing the density of states over the whole band. An analogous result is obtained for the hole concentration in the valence band, $\mathrm{p}$,

$$
\mathrm{p}=2\left(\frac{2 \pi \mathrm{m}_{\mathrm{p}}^{*} \mathrm{k}_{\mathrm{B}} \mathrm{T}}{\mathrm{h}^{2}}\right)^{3 / 2} \exp \left(\frac{\mathrm{E}_{\mathrm{F}}-\mathrm{E}_{\mathrm{C}}}{\mathrm{k}_{\mathrm{B}} \mathrm{T}}\right)=\mathrm{N}_{\mathrm{v}} \exp \left(\frac{\mathrm{E}_{\mathrm{V}}-\mathrm{E}_{\mathrm{F}}}{\mathrm{k}_{\mathrm{B}} \mathrm{T}}\right)
$$

where $\mathrm{NV}_{\mathrm{V}}$ is the effective density of hole states in the valence band.

A semiconductor whose properties are determined by the host atoms and not the impurities is an intrinsic semiconductor. The hole and electron concentrations in intrinsic semiconductors are equal. So, the product of Equations 2.7 and 2.8 yields

$$
\mathrm{pn}=\mathrm{n}_{\mathrm{i}}^{2}=\mathrm{N}_{\mathrm{C}} \mathrm{N}_{\mathrm{v}} \exp \left(-\frac{\mathrm{E}_{\mathrm{G}}}{\mathrm{k}_{\mathrm{B}} \mathrm{T}}\right)
$$

where $n_{i}$ is defined as the intrinsic carrier concentration and $E_{G}$ is the magnitude of the bandgap. Although the hole and electron densities are not equal in extrinsic semiconductors, the above equation still applies. This relation is known as the "law of mass action." The doping of a semiconductor shifts the Fermi energy from near the center of the bandgap for the intrinsic case to energies at or close to the level of the impurity. When acceptors are introduced, the Fermi energy approaches the valence band resulting in a greater concentration of holes than electrons. 
The carrier concentration in a semiconductor may exhibit both intrinsic and extrinsic behavior depending on the level of doping and the temperature range under observation. For example, at room temperature the intrinsic carrier concentration of $\mathrm{Ge}$ is of the order of $10^{13} \mathrm{~cm}^{-3}$; consequently, a doping level significantly below this concentration will not have an impact on the carrier density at room temperature. But $n_{i}$ has an exponential dependence with temperature and decreases very rapidly on cooling. Because the ionization energy of shallow hydrogenic impurities is so small compared to the bandgap, a temperature (below $300 \mathrm{~K}$ ) is reached at which the carrier concentration is mainly generated by the thermal ionization of dopants and not by the excitation of electrons across the bandgap. This temperature marks the beginning of the extrinsic region which is characterized by a constant carrier concentration over a broad temperature range. At a sufficiently low temperature, however, there is no longer enough thermal energy to fully ionize the impurity level, and the onset of the freeze-out region occurs.

The change in the hole concentration with temperature for p-type semiconductors with monovalent acceptors of binding energy $E_{A}$ is

$$
p=\frac{N_{A^{0}}}{N_{A^{-}}} \frac{N_{V}}{B} \exp \left(-\frac{E_{A}}{k_{B} T}\right) .
$$

$B$ is the spin degeneracy factor equal to 4 for the case of a fourfold degenerate valence band edge. If the semiconductor has $\mathrm{N}_{\mathrm{A}}$ acceptors and $N_{D}$ donors (per $\mathrm{cm}^{3}$ ) and $N_{A} \gg>N_{D}$, the ionized impurity concentration, $\mathrm{N}_{\mathrm{A}^{-}}$, is given by the sum of the hole concentration and the concentration of compensated centers: 


$$
\mathrm{N}_{\mathrm{A}^{-}}=\mathrm{p}+\mathrm{N}_{\mathrm{D}}
$$

The neutral acceptor concentration is the difference between the total and ionized acceptor concentrations:

$$
N_{A^{0}}=N_{A}-N_{A^{-}}=N_{A}-N_{D}-p \text {. }
$$

Thus, the hole concentration may be formulated as

$$
\frac{\left(p+N_{D}\right)(p)}{N_{A}-N_{D}-p}=\frac{N_{V}}{B} \exp \left(-\frac{E_{A}}{k_{B} T}\right) \text {. }
$$

The concentration of holes can then be derived from the Equation 2.11, which is a quadratic equation in p. However, some simplifications lead to less complicated approximations for different parts of the freeze-out region.

If all dopants are ionized, the net dopant concentration is $N_{A}-N_{D}$ for the present case. Consequently, during freeze-out the hole concentration is less than $\mathrm{N}_{\mathrm{A}}-\mathrm{N}_{\mathrm{D}}$. If $\mathrm{N}_{\mathrm{D}}<<<<\mathrm{N}_{\mathrm{A}}-\mathrm{N}_{\mathrm{D}}$,

$$
\frac{\left(p+N_{D}\right)(p)}{N_{A}-N_{D}-p} \approx \frac{p^{2}}{N_{A}} \approx \frac{N_{v}}{B} \exp \left(-\frac{E_{A}}{k_{B} T}\right) \text { and } p \approx \sqrt{\frac{N_{A} N_{V}}{B}} \exp \left(-\frac{E_{A}}{2 k_{B} T}\right)
$$

This is known as the half-slope region and is characterized by an Arrhenius relation with slope proportional to $E_{A} / 2$. If $p<<N_{D}$,

$$
\frac{\left(p+N_{D}\right)(p)}{N_{A}-N_{D}-p} \approx \frac{N_{D}}{N_{A}} p \approx \frac{N_{V}}{B} \exp \left(-\frac{E_{A}}{k_{B} T}\right) \text { and } p \approx \frac{N_{A} N_{V}}{N_{D} \beta} \exp \left(-\frac{E_{A}}{k_{B} T}\right) \text {. }
$$

Similarly, this is known as the full slope region since the change in the natural $\log$ of the hole concentration with inverse temperature is proportional to $\mathrm{E}_{\mathrm{A}}$. 
The change from half-slope to full slope occurs at $p=N_{D}$. It should be noted that the temperature dependence of the effective density of states was not considered; a plot of $\ln \left(\mathrm{pT}^{-3 / 2}\right)$ versus $1 / \mathrm{T}$ better represents an Arrhenius relation. In: addition, there may be other factors which affect freeze-out statistics (e.g., excited states, ground state splitting, etc.). These have been reviewed by Blakemore. ${ }^{19}$ Figure 2.3 is a theoretical curve following the above model for the case of p-type Ge with the specifics given in the figure.

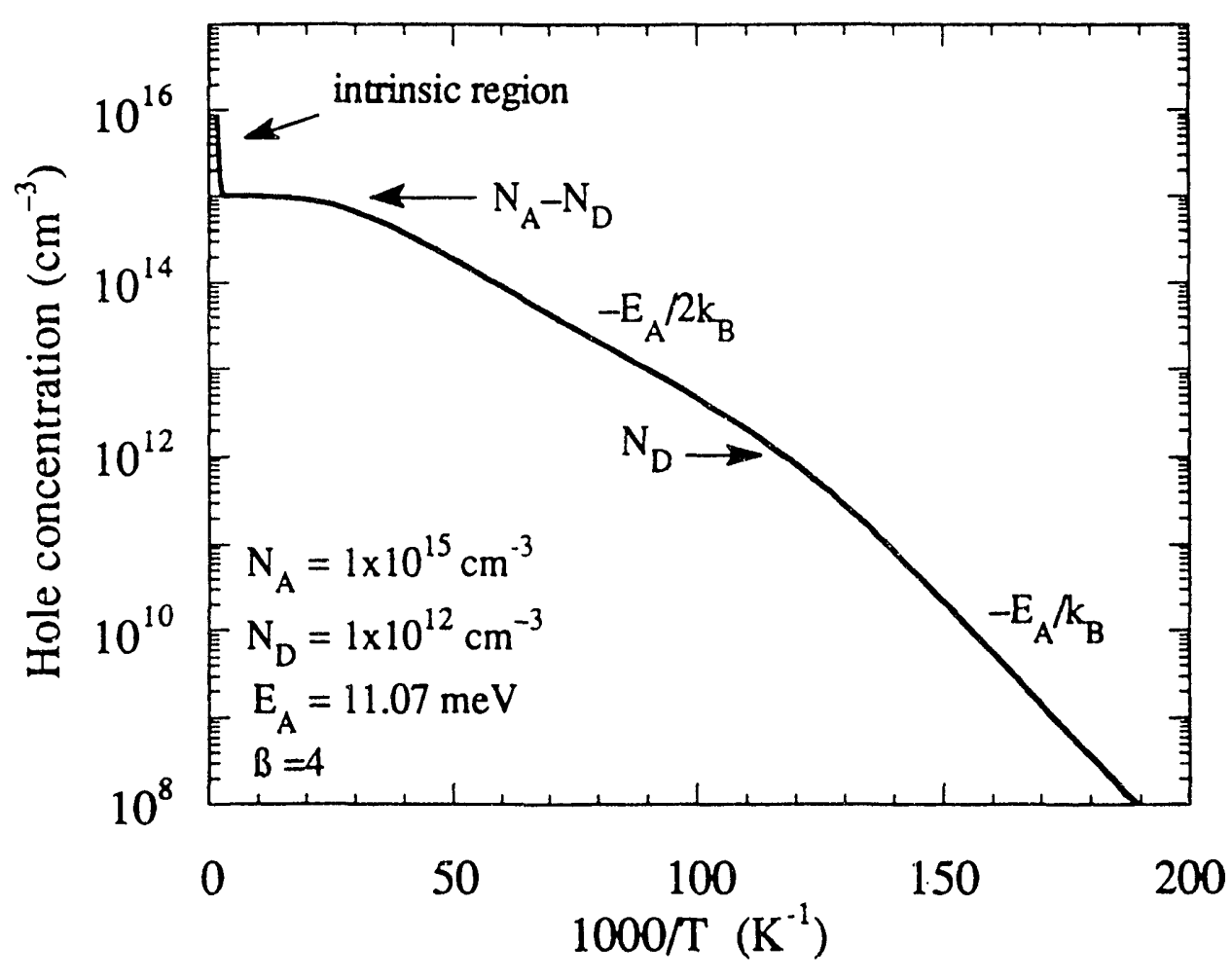

Figure 2.3. Theoretical curve for the freeze-out of holes in p-Ge. 
The statistics thus far discussed assumed monovalent impurities but the fundamental concepts do apply to divalent centers including $\mathrm{Be}$ in germanium. To a limited extent the two levels may be treated independently -as two different dopants of the same type. However, the centers must first be singly ionized in order for the deeper level to interact with either the band or with dopants of the opposite type. It is also clear that the carriers excited from the deeper level freeze-out at higher temperatures than carriers resuiting from singly ionizing the centers. If the minority impurity concentration is equal to or less than the concentration of the divalent majority impurities, only the shallower level will be compensated. The deeper level will undergo 1/2-slope freeze-out statistics. In practice this slope cannot be accurately determined since it occurs over a small range in concentration (i.e., between $2 \mathrm{~N}_{\text {majority- }}$ $\mathrm{N}_{\text {minority }}$ and $\mathrm{N}_{\text {majority }}-\mathrm{N}_{\text {minority }}$ ).

The Be-doped Ge samples used in this study have a compensation ratio, $N_{D} / N_{A}$, of about 0.001 . In turn, the freeze-out curve is very similar to that of shallow levels clearly showing half-slope and full-slope regions for the singly ionized state (with the appropriate impurity ionization energy). Ionization of $\mathrm{Be}^{-}$to $\mathrm{Be}^{--}$occurs at the higher temperatures and is not expected to have any significant impact on the physical processes of interest in the samples used for this study.

\subsection{Hole mobility in Ge}

When an external electric field $\mathbf{E}$ is applied to a semiconductor, the charge carriers experience a force given by $F=e E$. Yet the carriers do not accelerate continuously; rather, they undergo collisions with impurities 
and/or lattice vibrations which cause them to decelerate. This process is physically expressed in terms of a mean free path and a scattering time (also known as the time between collisions and the relaxation time). Under low field conditions the velocity of the carriers is proportional to the field applied, and the constant of proportionality is the materials parameter known as the electron (hole) mobility which is typically expressed in units of $\mathrm{cm}^{2} / \mathrm{Vs}$.

The three major scattering mechanisms that affect the mobility of carriers in a non-degenerately doped semiconductor are 1) phonon or lattice scattering, 2) ionized impurity scattering, and 3) neutral impurity scattering. The dominance of each mechanism depends on the temperature. Lattice scattering in doped $\mathrm{Ge}$ is significant above liquid nitrogen temperatures $(>77 \mathrm{~K})$ due to the increased density of acoustic phonons. As the temperature drops below seventy or eighty degrees Kelvin, scattering from ionized impurities becomes the dominant mechanism regulating carrier mobility in moderately to highly doped material. It is no surprise then that the dopant concentration plays a significant role, for every impurity center represents a potential scattering site. As the temperature is decreased, carrier freeze-out returns ionized impurities to their neutral state. So, the impact of ionized impurities on mobility should deminish with decreasing temperature. Such is the case in semiconductors with very low compensation; most impurities become neutral. This leads to the third scattering mechanism, that dominated by neutral impurities. Highly compensated, doped material, on the other hand, contains a significant number of impurities that remain ionized regardless of temperature, and neutral impurity scattering is not observed. 
In order to evaluate the impact of various scattering mechanisms on mobility, it is important to understand how each of these contribute to the relaxation time. Once a scattering time has been formulated, the mobility of carriers can be estimated:

$$
\frac{1}{\tau}=\sum_{i} \frac{1}{\tau_{i}} \quad \text { and } \quad \mu=\frac{e \tau}{\mathrm{m}^{*}}
$$

where the average overall time between collisions $\tau$ is equal to the inverse of the sum of the inverse of the relaxation times associated with each of the mechanisms. As can be seen from this sum, the mechanism yielding the smallest scattering time is dominant. Because the temperature range of interest in this study lies below $15 \mathrm{~K}$, scattering by impurities is of particular importance, but a brief summary of phonon scattering is presented as well.

The presence of lattice vibrations in a crystal results in small fluctuations in the spacing of atoms. This motion of the atoms leads to deviations from thie positions they would occupy in a rigid lattice and creates a "coarsening" of the band edges; that is, lattice vibrations manifest themselves as small band fluctuations. The charge carriers encounter these band ripples and have a finite probability of being reflected in an analogous manner to an electron encountering a step potential. This reflection can be interpreted as a scattering event and leads to a formulation of mobility $2 n$ that is proportional to $\mathrm{m}^{*-5 / 2 \mathrm{~T}-3 / 2}$.

Neutral impurity scattering is a low temperature process because it requires a significant fraction of the dopants to be neutral. For Ge this mechanism can become dominant below $20 \mathrm{~K}$. In general, it is usually difficult to observe neutral impurity scattering. As the free carrier 
corcentration decreases, the material's impedance increases significantly making electrical measurements difficult. If carrier scattering were dominated by neutral impurities, the carrier mobility would be independent of temperature, inversely proportional to the neutral impurity concentration, and proportional to the effective mass for shallow hydrogenic impurities. 13,21

Finally, in the temperature region where the density of phonons, (i.e., quanta of lattice vibrations) is low, ionized impurities are the most likely scatterers of charge carriers. The interaction between holes (electrons) in the valence (conduction) band and ions is analogous to the scattering of alpha particles, through a thin metal foil first analyzed in detail by Rutherford.2 ${ }^{22}$ A moving charge approaching a stationary charged particle will be detlected by the Coulomb interaction between the two charges. This change in trajectory crnstitutes a scattering event. In the case of a hole in a p-type semiconductor, this positive charge carrier experiences a repulsive force upon encountering an ionized (compensating) donor and an attractive force in the presence of a Coulomb field of an ionized acceptor. Regarciless of the type of interaction, the main result is that the trajectory of the hole changes, and the average time required to alter the carrier's forward trajectory by more than $90^{\circ}$ defines the relaxation time for this mechanism. The geometry and mathematical analysis of this physical problem produces a relaxation time approximately proportional to $\mathrm{T}^{3 / 2} \mathrm{~m}^{*} 1 / 2 \mathrm{Z}^{-2}$. Consequently, the mobility is proportional to $\mathrm{T}^{3 / 2} \mathrm{~m}^{*}-1 / 2 \mathrm{Z}^{-2}$. 


\subsection{Non-radiative capture of holes by acceptors}

A semiconductor material in thermal equilibrium maintains a concentration of electrons, $\mathrm{n}$, and holes, $\mathrm{p}$, which is determined by the statistical considerations discussed in Section 2.4. Just as carriers are generated in the bands at a particular rate, the inverse must occur. Carriers must be removed from the bands so as to maintain the thermodynamically favored carrier concentrations. In the case of extrinsic semiconductors, the carriers may leave the bands via recombination at impurity centers. This concept of the rate equality of a process and its inverse is known as the principle of detailed balance.

In the presence of an external excitation such as electromagnetic radiation and under steady-state conditions, a new balance between generation and recombination rates is established. In this case the sum of the thermal and external generation rates must equal the recombination rate,

$$
\mathrm{G}_{\mathrm{EXT}}+\mathrm{G}_{\mathrm{TH}}=\mathrm{R} \text {. }
$$

This simple expression can be rewritten for p-type semiconductors as

$$
N_{A} g_{A}+N_{A}{ }^{\circ} A_{A} N_{V} \exp \left(-\frac{E_{A}}{k_{B} T}\right)=p B_{A} B N_{A^{-}},
$$

where $g_{A}$ is the photon absorption rate by the neutral acceptor centers and $\mathrm{B}_{\mathrm{A}}$ is the recombination coefficient defined as the product of the free hole thermal velocity and the impurity center capture cross-section, $\sigma_{\mathrm{C}}$. Thus,

$$
p=\frac{G_{E X T}}{\sigma_{c^{v} \mathfrak{l}} B N_{A^{-}}}=G_{E X T} \tau_{p}
$$


and $\tau_{\mathrm{p}}$ is defined as the hole lifetime (or the majority carrier lifetime since acceptors are the majority impurities). The hole concentration at low temperatures is the product of the external generation rate and the lifetime.

The lifetime contains terms which are intricately woven into the physics involved in the capture of holes by acceptor centers. A hole travelling through a crystal at a velocity $v_{\text {th }}$ traverses a volume $A v_{\text {th }} t$ in the crystal over a time t.23 $A$ is the crystal's cross-sectional area normal to the hole's trajectory, and $v_{t h t}$ is the length of the hole's path. In this volume the hole potentially encounters $\left(v_{t h} t A\right) N_{A}-$ recombination centers. If the hole approaches the center at a distance $r_{c}$, it is captured. This event is assumed independent of the direction of approach. Therefore, there is an effective volume for capture around a recombination center equal to a sphere of radius $r_{c}$. If these spheres were projected onto a plane of area $A$, the fraction of the plane which may involve hole capture events is the sum of the cross-sectional areas $\left(\sum \sigma_{\mathrm{c}}\right)$ of the recombination centers divided by the total cross-sectional area. Consequently, the probability for capture is

$$
\frac{\sum\left(\sigma_{c}\right)_{i}}{A}=\frac{N_{A}-\left(v_{t h} t A\right) \sigma_{c}}{A}=N_{A^{-}}\left(v_{t h} t\right) \sigma_{c}
$$

and the rate of recombination $\mathrm{R}$ (the number of carriers captured per unit time) is

$$
\frac{\mathrm{pN}_{\mathrm{A}}-\left(\mathrm{v}_{\mathrm{th}} \mathrm{t}\right) \sigma_{\mathrm{c}}}{\mathrm{t}}=\mathrm{pN}_{\mathrm{A}^{-}} \mathrm{v}_{\mathrm{th}} \sigma_{\mathrm{c}}
$$

which is the same as the expression for recombination rate given by Equation 2.12 once the spin degeneracy factor has been taken into account. 
The major challenge in understanding the physics of carrier capture by an impurity is the conceptualization and subsequent quantification of the carrier capture cross-section. Yet this was not a new problem when first considered for the case of semiconductors. In $1924 \mathrm{JJJ}$. Thomson proposed a model for recombination in gases. 24 An ion can be captured by a Coulombic (attractive) center such as an oppositely charged ion if its energy is of the order of the thermal energy $3 k_{B} T / 2$. If the ion approaches the attractive center within a critical radius $r_{c r i t}$ and undergoes a collision with a third body (like a neutral atom), the ion will lose energy, and the Coulombic well will trap the ion into a state in which the thermal energy available to the ion $\left(\sim \mathrm{k}_{B} \mathrm{~T}\right)$ is insufficient to cause ion-center dissociation. The minimum condition for this is

$$
\mathrm{Ze}^{2} / \mathrm{rcrit}=3 \mathrm{k}_{\mathrm{B}} \mathrm{T} / 2
$$

where $\mathrm{Z}$ is the charge state of the center. The probability that a capturing collision within a sphere of radius $r_{c r i t}$ will occur is $4 \mathrm{rcrit} / 3 l$, where $l$ is the ion's mean free path and $4 / 3$ is an averaging factor. The crose ectional area of the sphere given by $\pi \mathrm{r}_{c r i t}{ }^{2}$. The product of these two terms defines Thomson's capture cross section, $\sigma_{\mathrm{T}}=4 \pi\left(\mathrm{r}_{\text {crit }}\right)^{3 / 3}$ l.

There are several mechanisms by which a hole that has been excited to the valence band can be captured and return to the acceptor ground state. The charge carrier may lose energy through a collision with another charge carrier (Auger process), the emission of a photon, the emission of phonons, or a combination of these processes. In his well known paper Melvin Lax ${ }^{25}$ first proposed that impurity centers exhibiting large capture cross sections $\left(\approx 10^{-14} \mathrm{~cm}^{2}\right)$ in $\mathrm{Si}$ and $\mathrm{Ge}$ trap charge carriers by a phonon cascade mechanism based on Thomson's model. For carriers with an equilibrium thermal distribution, the cross section $\sigma$ is given by 


$$
\sigma=\frac{\left\langle v_{0} \sigma\left(E_{0}\right)\right\rangle}{\left\langle v_{0}\right\rangle}=\frac{\int E_{0} \sigma\left(E_{0}\right) \exp \left(-\frac{E_{0}}{k_{B} T}\right) d_{0}}{\int E_{0} \exp \left(-\frac{E_{0}}{k_{B} T}\right) d_{0}}
$$

where $\mathrm{E}_{0}$ the carrier energy and the $\sigma\left(\mathrm{E}_{0}\right)$ is the cross-sectional term that is formulated based upon the appropriate capture mechanism. The latter can be formulated without losing any generality by an impact parameter calculation,

$$
\sigma\left(E_{0}\right)=\int 2 \pi b P_{c}\left(E_{0}, b\right) d b
$$

where $b$ is known as the impact parameter. $P_{c}\left(E_{0}, b\right)$ is the probability that a collision leading to capture will occur along an orbit that begins with an impact parameter $b$ and a particle energy $E_{0}$.

The general equations above can be specified for the case of cascade capture of holes by emission of acoustic phonons (which occurs at sufficiently low temperatures) in the following manner. A hole with kinetic energy $E_{0}$ approaches an attractive Coulombic center. The hole is a distance $b$ away from an imaginary line that is drawn through the capture center and parallel to the hole's path at a point where the energy due to center's Coulomb field is lower than the available thermal energy. At a time $t$ the hole is at a distance $r$ from the center and, under the influence of the Coulomb potential, is accelerated to a kinetic energy 


$$
E(t)=E_{0}-V[r(t)]=E_{0}+\frac{Z e^{2}}{\varepsilon_{0} \varepsilon_{r} r(t)}
$$

but still with a total energy of $E_{0}$. In order for a hole to be captured into a state of binding energy $U$, it must emit a phonon of energy $E_{0}+U$. This requires the kinetic energy $E$ to be greater than an energy $E_{m}=$ $\mathrm{ms}^{2} / 2\left[1+\left(E_{0}+U\right) /\left(2 \mathrm{~ms}^{2}\right)\right]^{2}$ where $s$ is the speed of sound in the material. The hole will achieve a kinetic energy of $E_{m}$ by approaching the attractive center at a distance $\mathrm{r}_{0}$ such that

$$
\frac{1}{2} \mathrm{~ms}^{2}\left[1+\frac{\mathrm{E}_{0}+\mathrm{U}}{2 \mathrm{~ms}^{2}}\right]^{2}=\mathrm{E}_{0}+\frac{\mathrm{Ze}^{2}}{\varepsilon_{0} \varepsilon_{\mathrm{r}} \mathrm{r}_{0}}
$$

Therefore,

$$
\mathrm{r}_{0} \approx \frac{16 \mathrm{Ze}^{2} \mathrm{~ms}}{2 \varepsilon_{0} \varepsilon_{\mathrm{r}}\left(\mathrm{E}_{0}+\mathrm{U}\right)^{2}}
$$

indicates the radius of the orbit necessary for capture into a state of binding energy $U$. In the crude approximation that the binding energies of greatest relevance are of the order of $k_{B} T$ (as assumed by Lax) and that the initial energy for a hole that is likely to be captured is also of the order of $k_{B} T$, then

$$
\sigma\left(\mathrm{E}_{0}\right) \approx \int 2 \pi \mathrm{b} \mathrm{db} \cdot \frac{\mathrm{r}_{0}}{l}
$$

and

$$
\sigma \approx \pi \mathrm{b}_{0}^{2} \frac{\mathrm{r}_{0}}{l}
$$


where $b_{0}$ is given by $r_{0}\left(E / E_{0}\right)^{1 / 2}$, and $l$ is the hole mean free path. This order of magnitude approximation is similar to Thomson's result but for a factor of $\mathrm{E} / \mathrm{E}_{0}$. This factor indicates that the particle's initial energy (and not just the particle's energy at the time of capture) plays a role in the formulation of the capture cross section. In the limit of $k_{B} T>>m s^{2}$,

$$
\sigma \approx \frac{4 \pi}{l}\left(\frac{\mathrm{Ze}^{2}}{\varepsilon_{0} \varepsilon_{\mathrm{r}} \mathrm{ms}^{2}}\right)^{3}\left(\frac{\mathrm{ms}^{2}}{\mathrm{k}_{\mathrm{B}} \mathrm{T}}\right)^{5} .
$$

The reader is referred to the original article 25 for the rigorous derivation of the capture cross-section from the Lax model. Nevertheless, this approximation reveals the functional dependence of $\mathrm{T}^{-4}$ (since $l$ is proportional to $\mathrm{m}^{*-2} \mathrm{~T}^{-1}$ ) of the cross-section given by Lax. The validity of the model has been evaluated at least in part by the comparison of the temperature dependence of the measured cross sections with the model.

Even the rigorous formulation set forth by Lax requires some fundamental assumptions. First, capture occurs largely at high bound excited states (having large Bohr radii) associated with the center. Consequently, the motion of the particle can be treated classically, and the energy levels are sufficiently close that they may be treated as a continuum of states. Second, the probability for capture, or sticking probability, is independent of the particle's angular momentum. Third, like Thomson, Lax assumed that only one collision is required for capture. This is the first of a two step process. The second step involves the particle transiting over the energy states and the sticking probability determining the likelihood that the hole will eventually reach the ground state. For the case of states of binding energy equal to $\mathrm{k}_{\mathrm{B}} \mathrm{T}$, the sticking probability is close to 
one. Also, Lax assumed in his model that the time required for the particle to diffuse through the states subsequent to the initial collision is small within the time scale of the observed photoconductive decay time ( $\approx$ carrier lifetime) regardless of whether the final result is capture to the ground state or re-emission into the band.

Revisions have been made to this model, $26-29$ the major one having been proposed by Abakumov et al.28,29 The Leningrad group proposed that particles of energy $\sim \mathrm{ms}^{2}$ and not $\mathrm{k}_{\mathrm{B}} \mathrm{T}$ were involved in the capture into states of binding energies of the order of $\mathrm{ms}^{2}$ (and not $\mathrm{k}_{\mathrm{B}} \mathrm{T}$ ). Thus, Lax underestimated the value of the sticking probability at binding energies of the order of $\mathrm{ms}^{2}$. In addition, the particles lose energy continuously (for the case $k_{B} T>>m s^{2}$ ) through the zero energy point (where bound states are of negative and band states are of positive energies). This yields a new expression for the capture cross section whose functional dependence on temperature is different in the energy range $k_{B} T>>m s^{2}$ and $k_{B} T<<m s^{2}$ :

$$
\begin{aligned}
& \sigma=\frac{4 \pi}{3 l}\left(\frac{2 \mathrm{~ms}^{2}}{\mathrm{k}_{\mathrm{B}} \mathrm{T}}\right)\left(\frac{\mathrm{Ze}^{2}}{\varepsilon_{0} \varepsilon_{\mathrm{r}} \mathrm{k}_{\mathrm{B}} \mathrm{T}}\right)^{3} \text { for } \mathrm{k}_{\mathrm{B}} \mathrm{T}>\mathrm{ms}^{2}, \\
& \sigma=\frac{16 \pi}{45 l \mathrm{~ms}^{2}}\left(\frac{1}{\mathrm{k}_{\mathrm{B}} \mathrm{T}}\right)^{2}\left(\frac{\mathrm{Ze}^{2}}{\varepsilon_{0} \varepsilon_{\mathrm{r}}}\right)^{3} \text { for } \mathrm{k}_{\mathrm{B}} \mathrm{T} \ll \mathrm{ms}^{2} .
\end{aligned}
$$

These may be combined into an interpolation equation:

$$
\sigma=\frac{4 \pi}{3 l}\left(\frac{2 \mathrm{~ms}^{2}}{\mathrm{k}_{\mathrm{B}} \mathrm{T}}\right)\left(\frac{\mathrm{Ze}^{2}}{\varepsilon_{0} \varepsilon_{\mathrm{r}} \mathrm{k}_{\mathrm{B}} \mathrm{T}}\right)\left(\frac{\mathrm{Ze}^{2}}{\varepsilon_{0} \varepsilon_{\mathrm{r}}\left(\mathrm{k}_{\mathrm{B}} \mathrm{T}+2.74 \mathrm{~ms}^{2}\right)}\right)^{2} .
$$


These equations show that the capture cross section is proportional to $\mathrm{T}^{-3}$ for $k_{B} T \gg>m^{2}$ and $T^{-1}$ for $k_{B} T<<m s^{2}$.

In addition to the modifications by Abakumov et al., attempts have been made to incorporate the quantum mechanical nature of bound excited states of hydrogenic impurities to the formulation of the capture cross section in n-type $\mathrm{Si}$ and Ge.30-32 The authors considered only the role of the six lowest, s-type, bound excited states on the capture process. In their analysis the total cross section is given appropriately by

$$
\sigma_{t}=\sum_{n=2}^{7} P_{n} \sigma_{d}(n)
$$

where $P_{n}$ is the sticking probability and $\sigma_{c}$ is the cross section for the bound excited state of quantum number $\mathrm{n}$. Below $10 \mathrm{~K}$ this quantum mechanical treatment provides an adequate temperature dependence but fails to give correct-order-of-magnitude values. A variety of efforts to fit the cascade model to the experimental values has achieved a varying degree of success. Recent results from Darken et al. 33 suggest that capture does not occur via a cascade process. A definitive presentation regarding the details of the mechanism for carrier capture by "giant traps" has yet to be presented.

\subsection{Hall effect and photo-Hall effect}

Several methods exist for determining the carrier lifetime in a material.34,35 The method chosen for this study was the photo-Hall effect measurement technique. The two concepts which form the basis for this method are the Hall effect ${ }^{36}$ and carrier statistics in the presence of an external generation source. The latter has already been described in 
Sections 2.4 and 2.6. This section briefly reviews the basics of Hall effect. More elaborate presentations on the subject may be found elsewhere. 37

In the presence of a magnetic field $\mathbf{B}$, a moving charge experiences a Lorentz force $F_{\mathbf{L}}$ defined by

$$
F_{\mathrm{L}}=\mathrm{q}(\mathbf{v x B})
$$

where $\mathbf{v}$ is the velocity of a carrier of charge q. In 1879 E.H. Hall ${ }^{36}$ observed that a metal strip carrying an electrical current $I_{X}$ and placed in a magnetic field $B_{Z}$ normal to the current direction exhibited a voltage $V_{H}$ perpendicular to both $I_{X}$ and $B_{Z}$. This is the Hall effect, and $V_{H}$ is known as the Hall voltage. The Hall effect is a direct manifestation of the production of a Lorentz force in a material by applied fields and the reaction of the material revealed as the Hall voltage. This phenomenon has since been used in the study of the nature and concentration of charge carriers not only in metals but also in other materials including semiconductors.

Figure 2.4 shows a bar of material which for this discussion is taken to be an extrinsic semiconductor. A current $I_{X}$ injected in the $+x$ direction results in the motion of mobile, charge carriers along the length of the bar with a drift velocity $v_{x}=I_{x} /(n q t w)$ where $n$ is the majority carrier concentration. (It is assumed that minority carriers contribute a negligible amount to electrical conduction.) If a magnetic field $B_{z}$ is applied in the $+z$ direction, the carriers will be deflected in the $-\mathrm{y}$ direction by a Lorentz force of magnitude $F_{L}=q v_{x} B_{z}$. As carriers accumulate on one side of the bar and are depleted on the other side, the redistribution of the mobile charge produces an electric field along the $y$ direction which prevents further accumulation of carriers. So, the Lorentz force is balanced by a 
field induced force $\mathrm{F}_{\mathrm{E}}$ equal to $\mathrm{qE}_{\mathrm{H}}$. In a homogeneous material under a uniform magnetic field, $E_{H}=V_{H} / w$. Thus,

$$
q v_{x} B_{z}-q E_{H}=q\left(\frac{I_{x}}{n q t w}\right) B_{z}-q \frac{V_{H}}{w}=0
$$

and

$$
\mathrm{qn}=\frac{\mathrm{I}_{\mathrm{X}} \mathrm{B}_{\mathrm{Z}}}{\mathrm{t} \mathrm{V}_{\mathrm{H}}}=\mathrm{R}_{\mathrm{H}}^{-1}
$$

where $R_{H}$ is the Hall coefficient. Because $I_{x}, B_{Z}, t$ and $V_{H}$ can usually be determined experimentally, the Hall coefficient is readily computed.
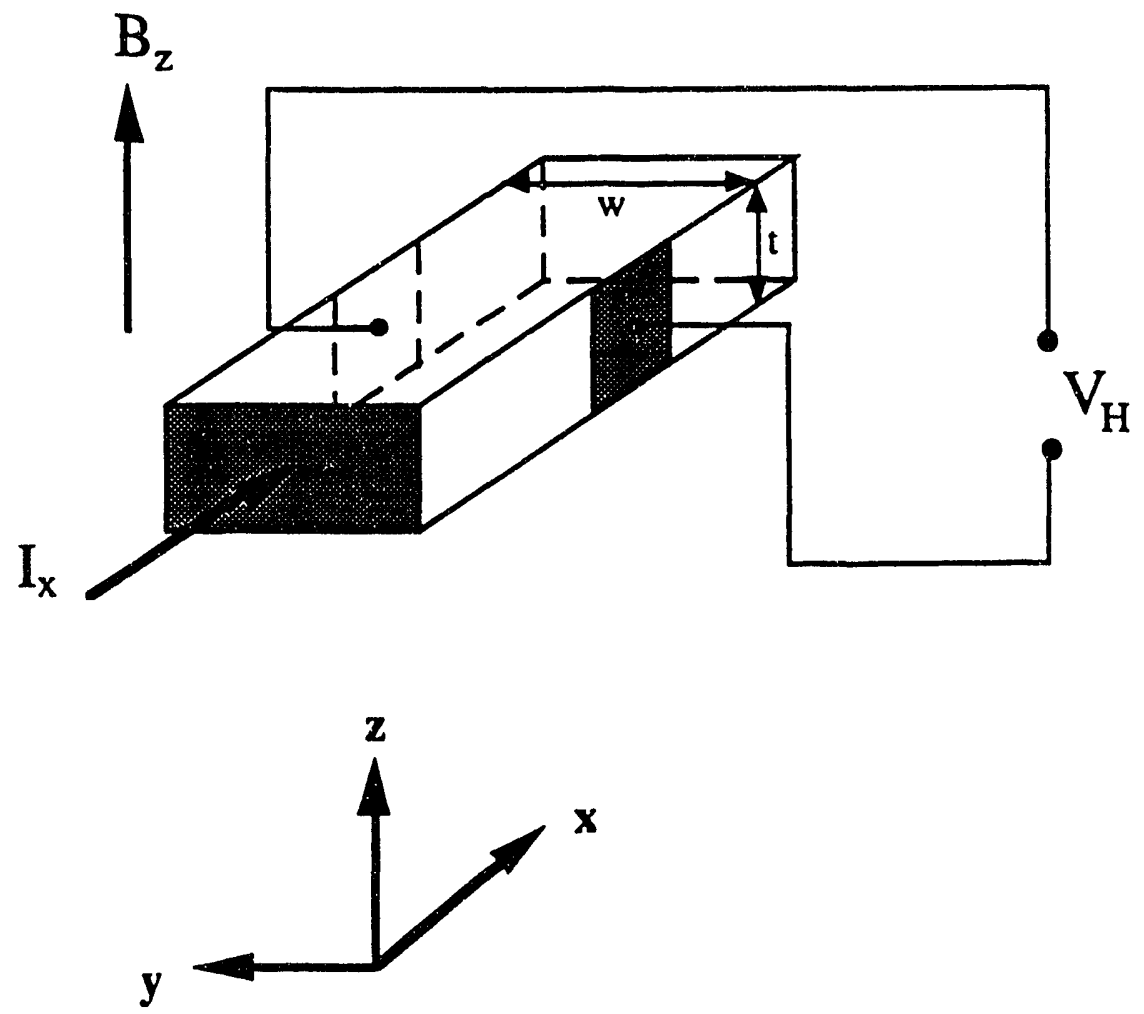

Figure 2.4. Schematic arrangement of a Hall measurement. 
The final result above (Eq. 2.24) elucidates the two features that make the Hall effect important for semiconductor characterization. The knowledge of the value of the Hall coefficient provides at once the majority carrier concentration and carrier type in a material. If $\mathrm{R}_{\mathrm{H}}$ is negative, $\mathrm{q}=$ e which means that electrons are the majority charge carriers; if $q=e$, then $R_{H}$ is positive and the material is p-type. The majority carrier concentration is determined from the magnitude of the Hall coefficient $\left(n=1 / R_{H} q\right)$; consequently, the measurement of the Hall coefficient as a function of temperature yields a profile of concentration versus temperature that generally obeys the freeze-out statistics covered in Section 2.4. The temperature dependent Hall measurements can allow for the determination of the majority and minority impurity concentrations and may provide information about the ionization energy of the majority impurity level.

It is found in practice that $R_{H^{-1}}$ is not exactly equal to the product of the charge and the carrier concentration. In general, $R_{H}=r_{H} / q n$ where $r_{H}$ is called the Hall factor which is a function of the relaxation time $\tau$ (defined in Sec. 2.5). Values for the Hall factor range from 1 to 2 and depend on the dominant carrier scattering mechanism(s). However, at high magnetic fields $r_{H}$ approaches unity. It has been shown ${ }^{38}$ that for p-type $G e$ at low temperatures, a magnetic field of $1 \mathrm{kG}$ is sufficient to meet the condition $\mu B>1$ ( $\mu$ is the carrier mobility in $m^{2} / \mathrm{Vs}$ and $B$ is in units of Tesla) reducing $\mathrm{r}_{\mathrm{H}}$ to approximately 1 .

The photo-Hall effect is very similar to the Hall effect phenomenon described above. The only difference is that carriers are generated optically as well as thermally. The temperature region of interest for determining the carrier lifetime is that in which optical generation 
dominates. In this case one may refer to the discussion regarding the influence of external generation sources on the carrier concentration and arrive at the relation

$$
\mathrm{R}_{\mathrm{H}}^{-1}=\mathrm{qn}=\mathrm{qG}_{\mathrm{EXT}} \tau_{\mathrm{n}} \text {. }
$$

Thus, the determination of the Hall coefficient in conjunction with the knowledge of the external (optical) generation rate yields the majority carrier lifetime (assuming that the photons are of sufficient energy to ionize impurities and not to produce electron-hole pairs due to band to band excitation).

\subsection{Effects of uniaxial stress}

The application of uniaxial stress to Ge has the effect of changing the character of the energy bands, 39,40 thus altering both the electrical properties of charge carriers and spectroscopic features of impurities in the material. When $\mathrm{Ge}$ is compressed along one of its principal crytallographic directions, the fourfold degeneracy of the valence band edge is broken leaving two, doubly degenerate split bands (along with the split off band which remains doubly degenerate below them). In the absence of stress, the valence band edge transforms according to the $\Gamma_{8}{ }^{+}$ irreducible representation of the $\mathrm{O}_{\mathrm{h}}$ point group. Compression along a $<100>$ direction reduces the crystal symmetry to $D_{4 h}$ and splits the $\Gamma_{8}{ }^{+}$ representation to the $\Gamma_{6}{ }^{+}$and $\Gamma_{7}+$ irreducible representations of the group $\mathrm{D}_{4 \mathrm{~h}} \cdot 40,41$ Similar group theoretical arguments have been provided for the splitting of bound states of hydrogenic acceptors. ${ }^{40-42}$ The actual energy 
shifts and splittings have been determined both experimentally9,16,42,43 and theoretically, $39,42,44,45$

The motion of the $\Gamma_{6}{ }^{+}$and $\Gamma_{7}+$ bands with respect to the unstressed valence band edge differs. Under compression the $\Gamma_{7}{ }^{+}$or "heavy hole" band shifts into the bandgap while the "light hole" band moves in the opposite direction on the energy scale. Similar behavior occurs with the ground state split levels of acceptors. In the unstressed condition the impurity ground state transforms as the $\Gamma_{8}$ irreducible representation of the $T_{d}$ point group. Uniaxial stress splits the ground state into the $\Gamma_{7}$ and $\Gamma_{6}$ irreducible representions of the $D_{2 d}$ point group, and their motion in energy space with stress is analogous to that of the valence band edge. To first order the rate at which the "heavy hole" band shifts with respect to uniaxial pressure is larger than that of the acceptor ground state. Thus, the ionization energy of the acceptor center decreases with increasing stress and may be reduced by as much as $5 \mathrm{meV}$ from its unstressed value. ${ }^{43}$

In addition to these energy changes, the motion as well as the density of states of carriers in the bands is significantly altered with stress. This is reflected in the different values of the effective masses. When a large compression is applied along one of the $\langle 100\rangle$ directions the nearly spheroidal constant energy surfaces of the valence band become ellipsoidal with the longitudinal effective mass achieving a smaller value than that of the transverse mass (oblate ellipsoids). 39,40 If the stress is of the tensile kind, the ellipsoid is prolate in shape; that is, the curvature of the band in the direction of stress is lower than that in an equivalent crystallographic direction which is normal to the stress. The effective mass is then given by

$$
\mathrm{m}^{*}=\left(\mathrm{m}_{\|} \mathrm{m}_{\perp}{ }^{2}\right)^{1 / 3},
$$


and the motion of carriers becomes anisotropic. The density of states which exhibits an effective mass dependence changes as well.

The piezoresistance of $\mathrm{Ge}$ was first reported by Smith. ${ }^{46}$ This provided evidence that the application of uniaxial stress to this semiconductor altered the electrical properties of the material. The use of cyclotron resonance and the quantum mechanical analysis of the deformed energy bandis ${ }^{17.49}$ provided a fundamental physical basis for such piezoresistive behavior. Finally, in $1960 \mathrm{~J}$. J. Hall ${ }^{9}$ performed Hall effect measurements on uniaxially stressed, p-type Ge and showed that both the mobility of heles is anisotropic and the ionization energy of the hydrogenic acceptor centers ( $\mathrm{Al}$ and $\mathrm{In}$ ) decreases with inc easing compressive stress. Samples under [100]-oriented stress and whose measurements were perfomed with the electrical current flowing in the stress direction exhibited an increase in the Hall mobility of a factor of two from the unstressed condition. 


\section{Experimental}

\subsection{Hall apparatus and measurement}

Only three pieces of equipment are needed to perform a Hall effect measurement: a) a volt meter, b) a current source, and c) a magnet. Fig. 3.1 is a schematic of a three component system with the additional features of automation and temperature control. The sample temperature was measured with a Lakeshore DRC 80 cryogenic thermometer when the standard coldfinger was used. The stressing coldfinger requires the use of a multimeter in order to read the resistance of a calibrated temperature sensing resistor.

Samples were mounted to a coldfinger attached to a Lakeshore CT310 continuous flow cryostat (Fig. 3.2). This allowed for Hall measurements at temperatures ranging from $300 \mathrm{~K}$ down to approximately $2.5 \mathrm{~K}$. Appropriate vacuum ( $10^{-5}$ torr) was reached via a diffusion pump system, and sub-liquid He temperatures were achieved by pumping on the He reservoir. Although optical ports are available for external photon sources, photo-Hall measurements were performed using an internal blackbody source as will be described in the following section.

When samples in the van der Pauw configuration ${ }^{50}$ were used, the determination of the Hall coefficient and the resistivity involved sixteen measurements, eight for each parameter. Although ideally only two measurements are required for each, eight were performed in order to minimize the effects of any voltage offsets (e.g. offsets at the contacts) and magnetoresistive effects in the case where a magnetic field was necessary. Because uniaxial stress induces anisotropic effects in the electrical 


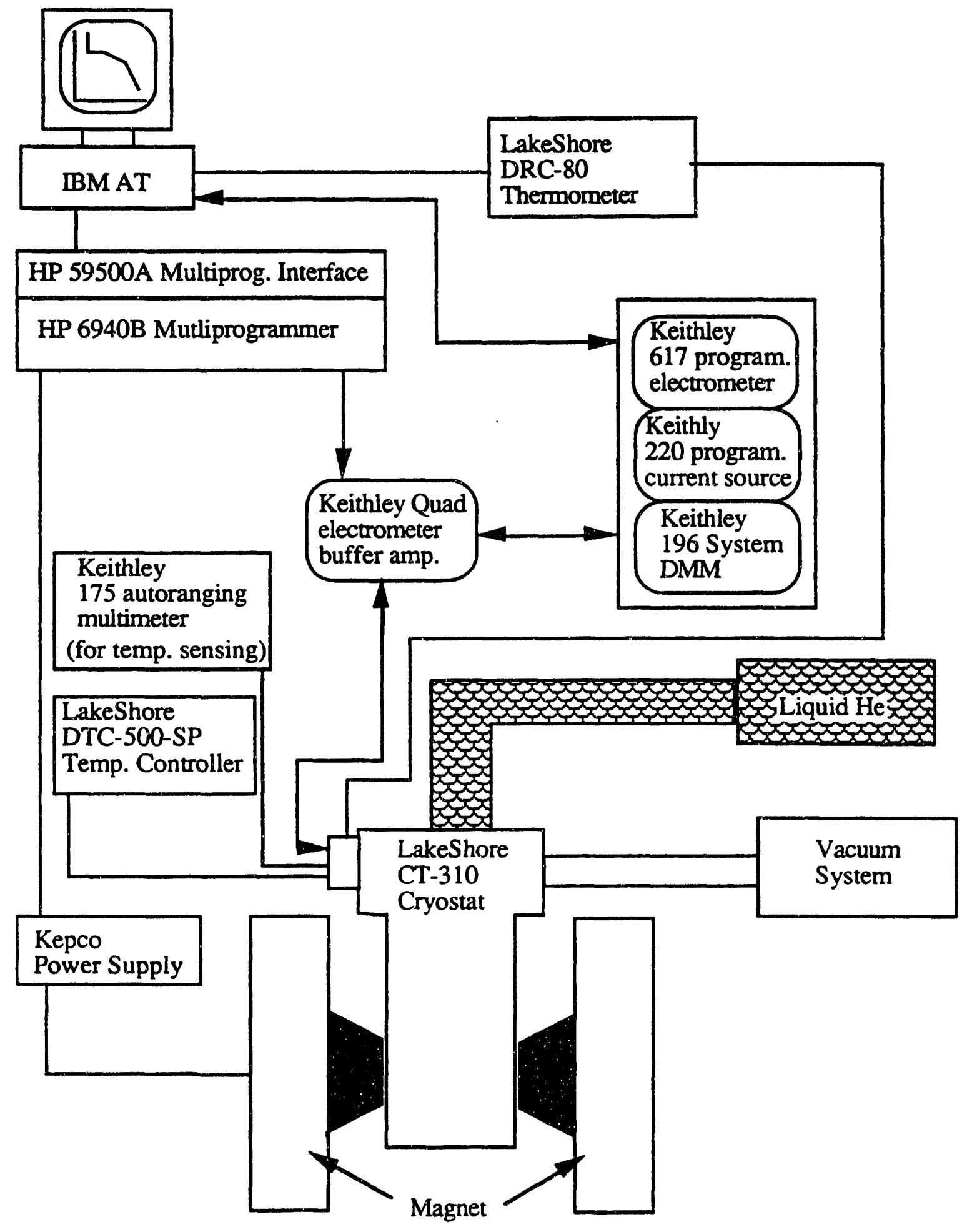

Figure 3.1. Schematic of Hall effect apparatus. 


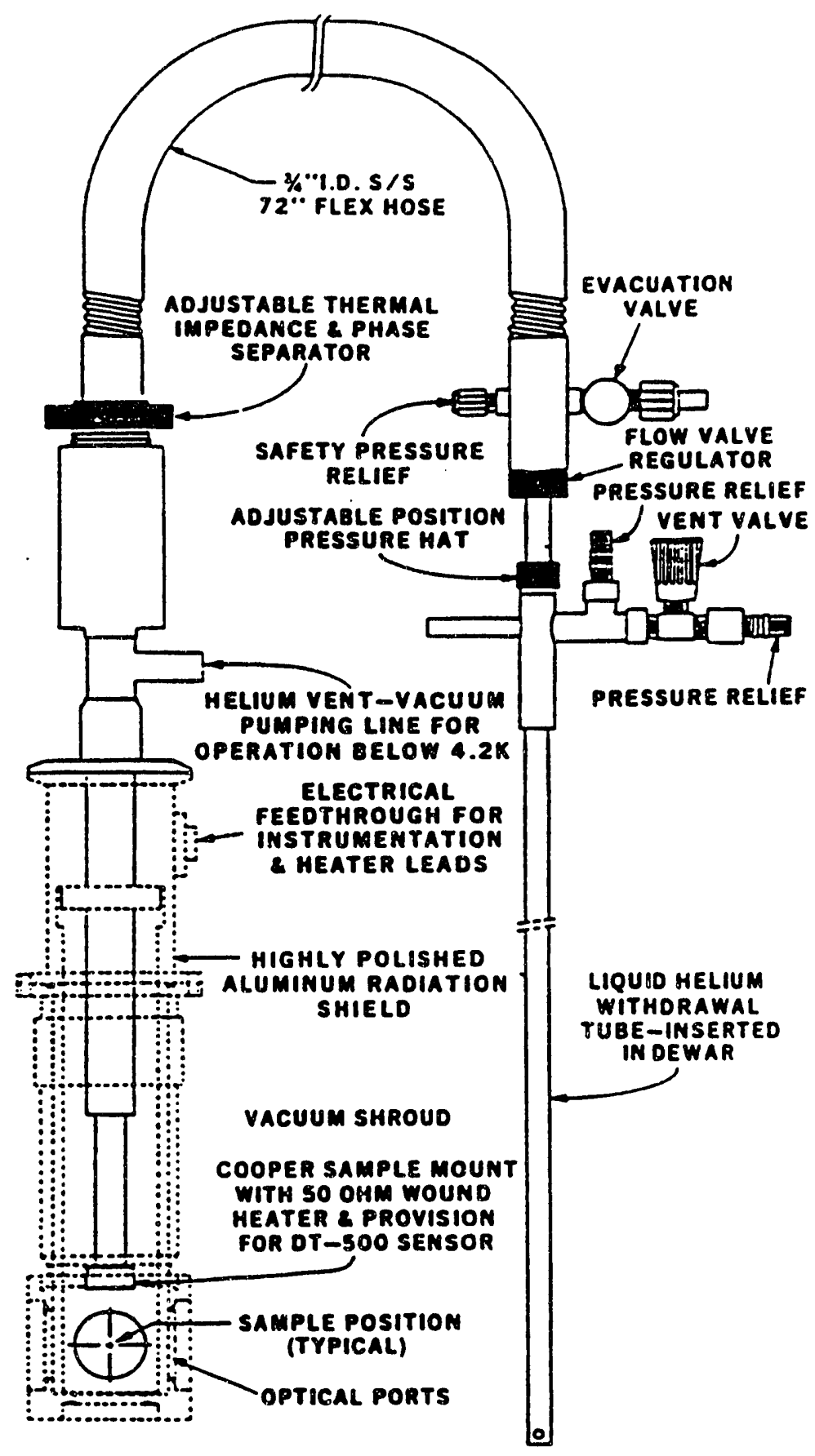

Figure 3.2. Schematic of Lake Shore CT-310 continuous flow cryostat. 
properties of most crystalline materials, a modified bar configuration was used for all stressed samples as shown in Figure 3.3. Current was passed through contacts 1 and 3 in either direction, and all voltages were measured across 2 and 4 . The sample's resistivity was determined by two measurements, one with current injected at contact 1 and the other at 3 . This provided a measure of the sample resistance $\left(\mathrm{V}_{\text {resist }}=0.5 \mathrm{~V}_{24} / \mathrm{I}_{13}\right.$ $\left.+0.5 \mathrm{~V}_{24} / \mathrm{I}_{31}\right)$. This resistance was converted to a resistivity by taking into account a geometric factor determined by obtaining the actual resistivity of samples configured in the van der Pauw geometry. This geometric factor may be calculated at one temperature and applied to other temperatures.
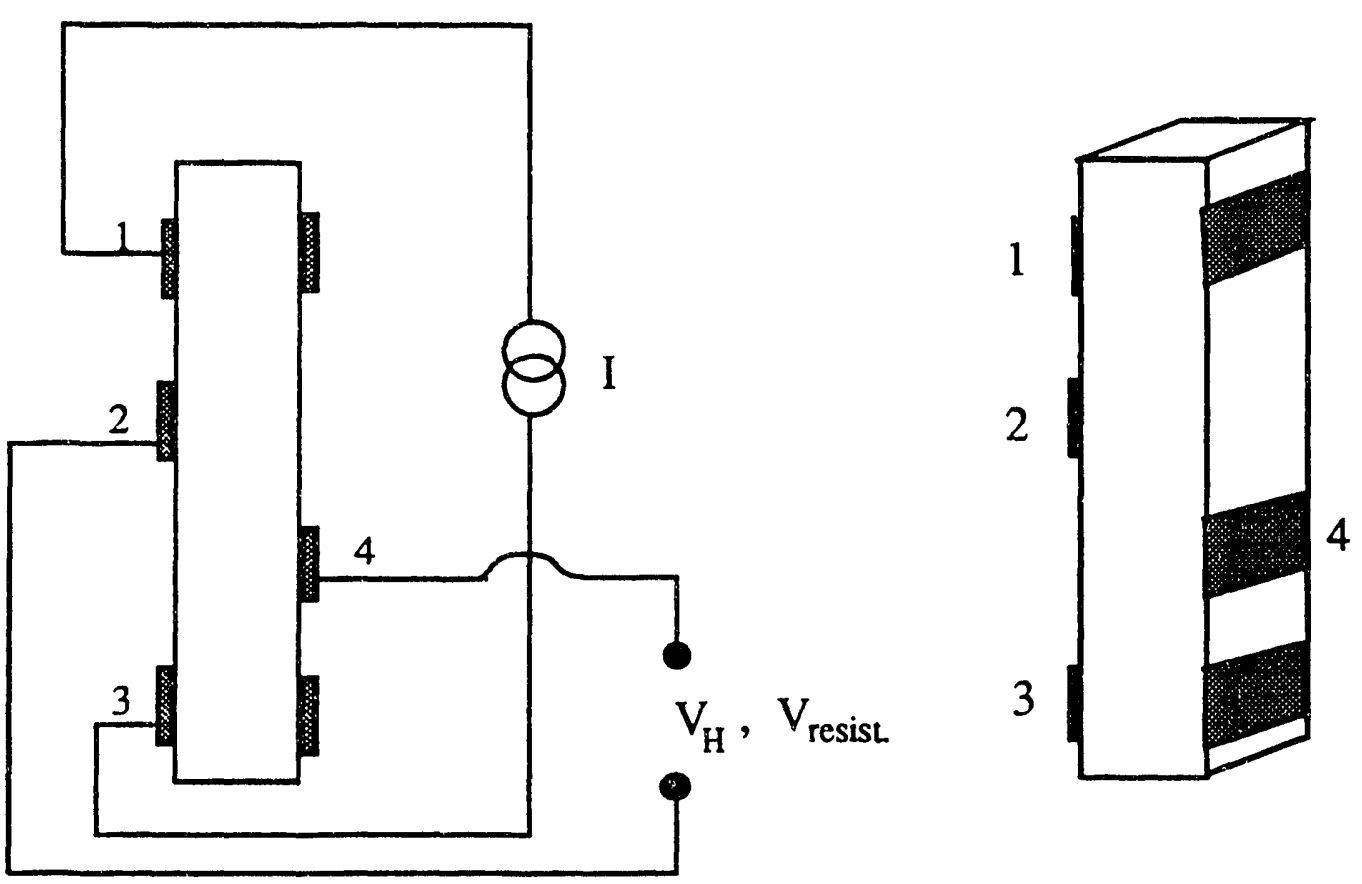

$1 \mathrm{~mm}$

Figure 3.3. Sample configuration for dark- and photo-Hall measurements. 
The Hall coefficients for the modified bar samples involved four rather than two measurements. The same arrangement as the one just describe was utilized with the additional requirement of a magnetic field. The magnet was set at a magnetic induction of 3 kGauss. Two measurements were performed with the field in one direction (normal to both the contactless surfaces and the axis of compression) and repeated with the field changed to the opposite direction-the measurements being averaged. By switching the field direction, one obtained the Hall voltage from the voltage measurements between contact 2 and 4 :

$$
\mathrm{V}_{\mathrm{H}}=\frac{\mathrm{V}_{\text {resist }}^{+}-\mathrm{V}_{\text {resist }}^{-}}{2}
$$

where the "+" and "-" superscripts indicate the orientation of the field along the $z$ axis (Fig. 2.4). The only sample dimension that was necessary to obtain the carrier concentration is the distance between the two contactless surfaces (equivalent to the dimension $t$ in Figure 2.4).

\subsection{Stressing coldfinger}

The characteristics of the stressing coldfinger are most important to the successful performance of standard and photo-Hall measurements of uniaxially stressed samples. Three factors were considered in its design: a) the accurate measure of temperature at low temperatures, b) the ability to apply uniaxial compression to small, bar shaped samples $\left(\approx 1 \times 1 \times 5 \mathrm{~mm}^{3}\right)$, and c) the capability of shining light on the sample. The major overall constraint in the design was space $(\approx 1$ inch cubed available). In addition, the coldfinger materials had to be of sufficient a) strength to support 
stresses of up to $6 \mathrm{kbars}\left(60 \mathrm{~kg} / \mathrm{mm}^{2}\right)$, b) thermal conductivity to minimize thermal time constants and c) non-magnetic behavior so as to not affect the Hall measurement.

A schematic of the stressing coldfinger is provided in Fig. 3.4. Its design was based on the stressing devices used successfully for low temperature, far-infrared photoconductor applications. 51 It includes the following components: a) the main body constructed from a solid piece of brass, b) one stainless steel piston and sample set screw, c) one \#4-40 screw with which to apply stress, d) one stainless steel fulcrum attachment, e) one calibrated temperature sensing resistor, f) one gold-coated brass, hemispherical, optical cavity, g) one cavity as described in $f$ ) and with a blackbody emitter within it, and h) one brass attachment used to connect the coldfinger to the Lakeshore cryostat. In addition, stress was applied via phosphor-bronze, leaf springs of various thicknesses.

\subsubsection{Temperature sensing resistor}

An Allen-Bradley, $1 / 8$ watt, $1 \mathrm{k} \Omega$, carbon composite resistor was used as the temperature sensor for the stressing coldfinger. After being cycled several times from room temperature to $4.2 \mathrm{~K}$, the resistor was calibrated between $77 \mathrm{~K}$ and $4.2 \mathrm{~K}$ with respect to the DRC-80 unit. All calibration points involved the measurement of resistance with a Keithley multimeter versus the temperature of the bath into which the resistor was placed. The $77 \mathrm{~K}$ and $4.2 \mathrm{~K}$ values were obtained with liquid nitrogen and liquid helium baths, respectively, at standard pressure. Measurements below 4.2 Kelvin were made by evacuating the liquid $\mathrm{He}$ space thus 


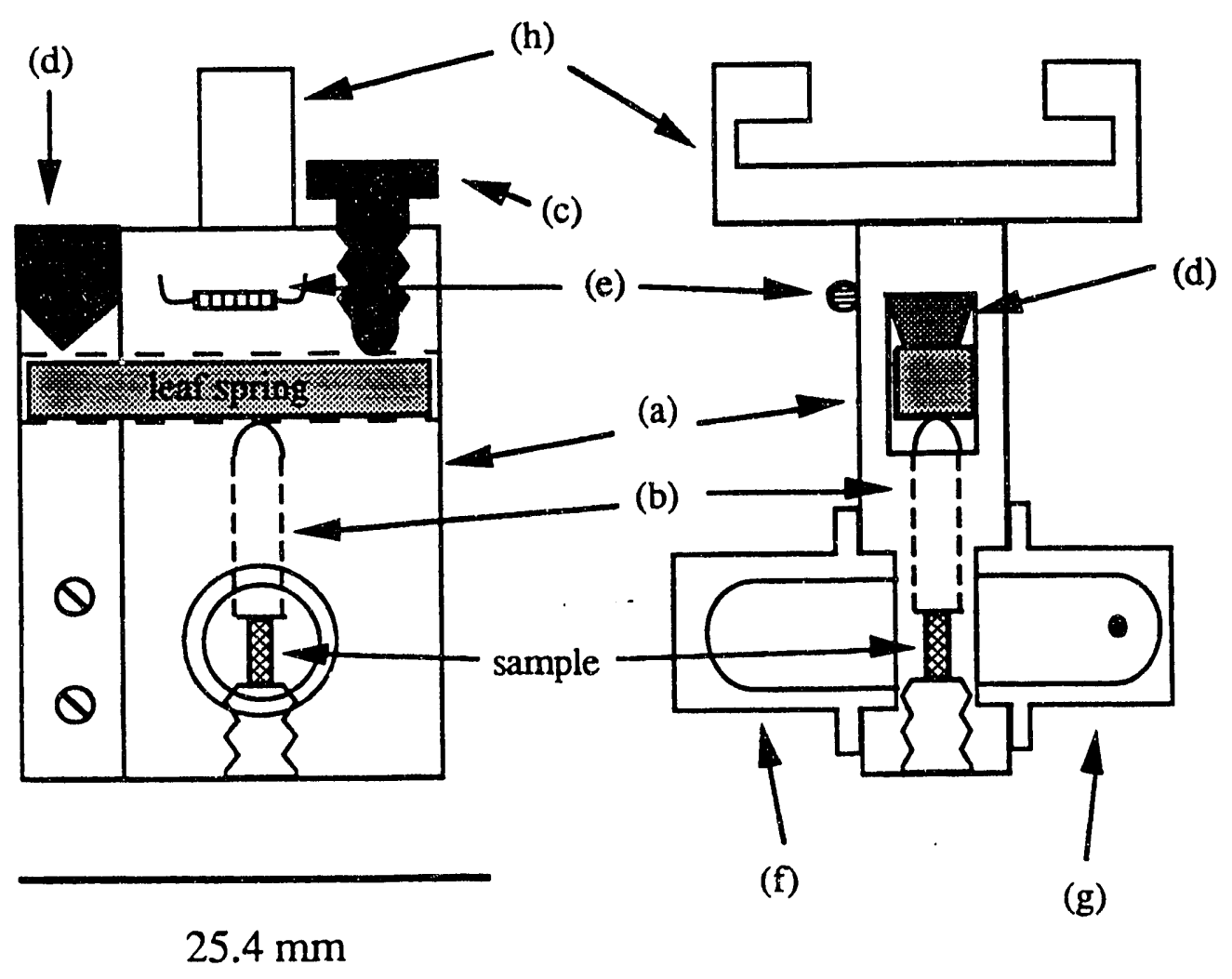

Figure 3.4. Drawing of the stressing coldfinger. All labeled components are identified in the text.

reducing the temperature of the bath. The relationship between pressure and liquid helium temperature is well known. This information was used to calibrate the resistor by making resistance versus pressure measurements. All Hall measurements were made with the same multimeter in order to ensure reproducibility of the temperature reading.

For resistors used over a relatively wide temperature range (3-20 $\mathrm{K})$, several empirical formulae to fit the temperature-resistance relation of Allen-Bradley resistors have been proposed. ${ }^{52}$ The data in the present case were fit with a fourth order polynomial $y=A+B x+C x^{2}+\mathrm{Dx}^{3}+\mathrm{Ex}^{4}$, where $\mathrm{x}$ 
is the natural logarithm of resistance (in units of ohms) and y is 1000/T (in units of $\mathrm{K}^{-1}$ ). The values for the constants are given in the table below.

\begin{tabular}{|c|c|}
\hline Constant & Value \\
\hline$A$ & 5169.19 \\
\hline$B$ & -2237.89 \\
\hline C & 349.817 \\
\hline$D$ & -23.5618 \\
\hline E & 0.601 \\
\hline
\end{tabular}

Table 3.1. Constants of the fourth order polynomial used to fit the resistance-temperature characteristics of a $1 \mathrm{k} \Omega$, Allen-Bradley resistor.

Figure 3.5 is a plot of $y$ versus $x$. The resistance of the $1 \mathrm{k} \Omega$ resistor begins to change significantly at low temperatures $(<30 \mathrm{~K})$. Therefore, this calibration allows for temperature measurements to within two or three percent at temperatures below $25 \mathrm{~K}$, the accuracy increasing as the temperature is decreased even further. Subsequent to the calibration process, the resistor was mounted with stycast to a small copper plate such that only the leads were exposed in order to minimize the effects of humidity and provide greater structural integrity. The small copper plate was then mounted directly to the coldfinger.

3.2.2 The blackbody emitter and other optical considerations

A knowledge of the carrier generation rate is essential to the determination of the carrier lifetime by the photo-Hall technique. To this end the optical aspects of the stressing coldfinger were designed with two 
objectives in mind: a) the capability of quantifying the spectrum of photons incident on the sample and $b$ ) the ability to determine the number of photons absorbed by the sample. Because shallow impurities in Ge have ionization energies of $\sim 11 \mathrm{meV}$ (or $\sim 110 \mu \mathrm{m}$ ), a far-infrared photon source was required. And because samples were measured under stress, the ionization energies were shifted to lower energies requiring either a tunable or broad bandwidth source.

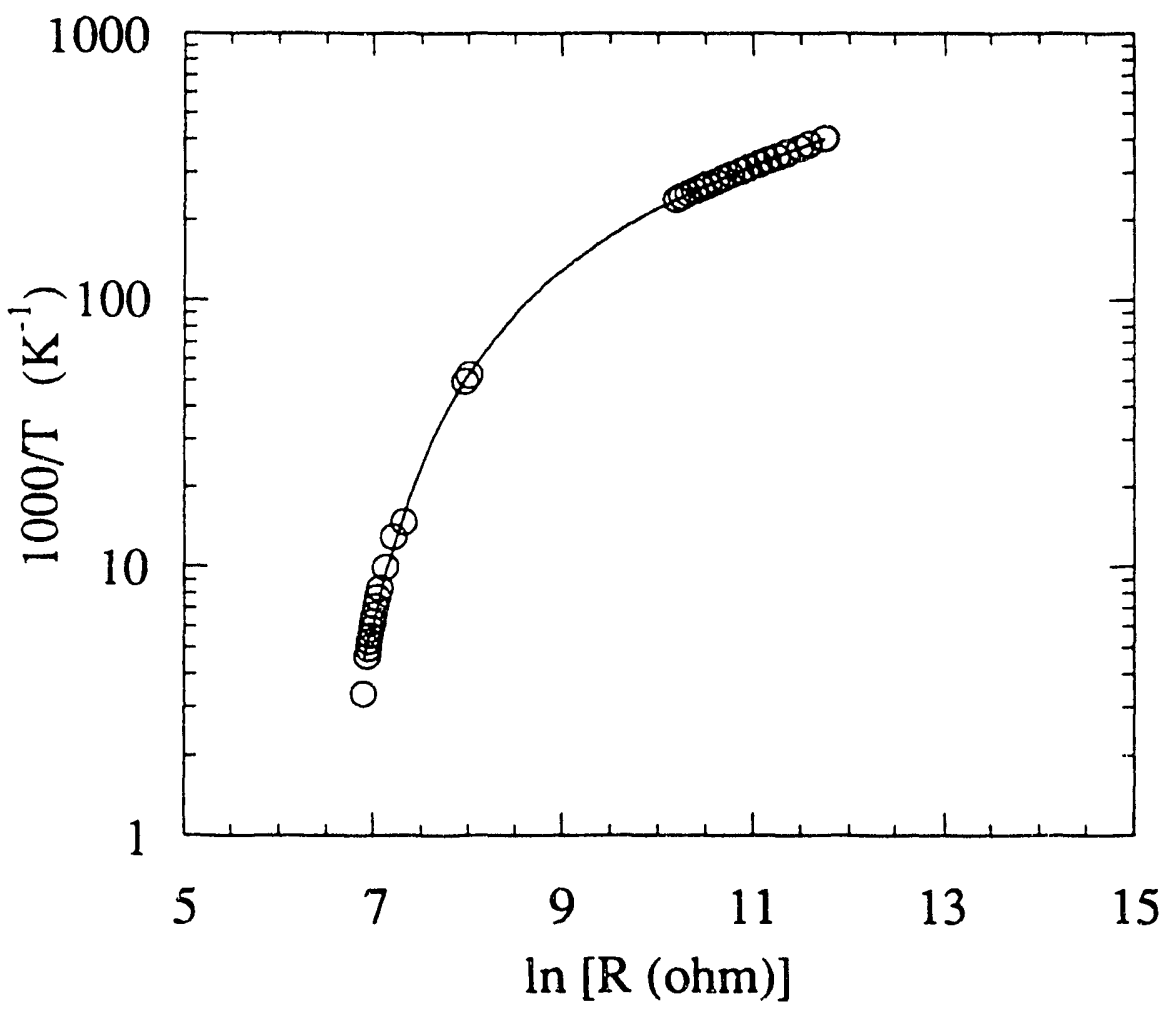

Figure 3.5. Fitting of temperature-resistance data of an Allen-Bradley, 1/8 watt, $1 \mathrm{k} \Omega$, carbon composite resistor with a fourth order polynomial and the related constants (Table 3.1). 
In the case where a broad bandwidth source is considered, it is necessary to know the absorption cross section of each impurity type as a function of photon energy. One can then compute the rate of photon absorption and, hence, the rate of carrier generation assuming that only one center is ionized per photon absorbed. This is not a bad assumption if the majority of photons are of low enough energy so as to not cause, for example, highly excited free carriers that ionize other centers.

These experimental challenges were met by the design of a closed cavity containing both the sample and a blackbody emitter (Figure 3.4). Two hemisphere-like cavities were machined from brass and their interiors were sputtered with gold. The emitter is constructed from a $\sim 1 \mathrm{k} \Omega \mathrm{NiCr}$ square chip one milimeter on the side. The leads are made of $25.4 \mu \mathrm{m}$ brass and are attached to the chip via silver epoxy. Once the leads were attached, the chip was painted with stycast epoxy such that an approximately spherical shape (diameter $\sim 1.4 \mathrm{~mm}$ ) was achieved. The leads run out of one of the cavities through small diameter teflon tubing. The tubing was mounted through a small hole on the side of the cavity and secured with stycast outside the cavity and silver epoxy inside the cavity.

Photo-Hall measurements were typically made below $20 \mathrm{~K}$. At these temperatures a fraction of the emitter's power was dissipated radiatively when current was injected through it. The temperature of the emitter was raised above that of its surrounding. It has been assumed that at the wavelengths of interest ( $>30 \mu \mathrm{m}$ ) the emitter behaved as a blackbody so This distribution of the photons radiated followed a Planck distribution which is only depends on the blackbody's temperature and for a $1.4 \mathrm{~mm}$ diameter sphere is given by 


$$
\mathrm{P}=\frac{1.16 \times 10^{4}}{\lambda^{4}\left[\exp \left(\frac{\left.1.44 \times 10^{-2}\right)}{\lambda \mathrm{T}} \cdot 1\right]\right.} \quad \text { (photons } / \mathrm{m}-\mathrm{s} \text { ) }
$$

where $\lambda$ is in units of meters and $\mathrm{T}$ is in units of Kelvin. Therefore, it was very important to know with some accuracy the temperature of the emitter. This was determined for three different currents and was measured with a half mil, Type $\mathrm{K}$ thermocouple and an HP3478A multimeter. The thermocouple was placed in contact with the emitter through a very small amount of GE varnish. Thus the light source was calibrated for three temperatures $77 \mathrm{~K}, 99 \mathrm{~K}$, and $122 \mathrm{~K}$.

The goal of constructing and calibrating the emitter was to provide the best possible quantification of the photon spectrum impingent on the sample, (i.e., the first objective). Using the two cavities to enclose both the sample and the emitter would facilitate quantifying the photon absorption rate by establishing conditions which woulc best arrive at a quantum efficiency of one. To a first approximation all photons incident on the sample that can be absorbed will be absorbed. Once steady state is reached, carriers are generated at a rate similar to that with which the photons are emitted by the blackbody. This approximation implies that the time required for the absorption of photons (including photons that are initially reflected by the sample surface) is small compared to the lifetime of the photoexcited holes.

The approximation of a quantum efficiency of one can be improved by accounting for the fraction of photons that is absorbed by surfaces within the cavity other than the sample surfaces. ${ }^{52}$ In this case the fraction of photons absorbed by the sample is 


$$
\frac{A_{\text {sample }} \alpha_{\text {sample }}}{\sum_{i} A_{i} \alpha_{i}}
$$

where $A_{i}$ is the area of an absorbing surface within the cavity, and $\alpha_{i}$ is the corresponding absorptivity which depends on the photon energy. This quantity is difficult to calculate due to the limited data available regarding the absorptivities ( $\alpha_{\text {samples's) }}$ ) of $\mathrm{Ge}: \mathrm{Ga}$ and $\mathrm{Ge}: \mathrm{Be}$ as a function of photon energy. However, it is important for the reader to know that absorption by other surfaces may have reduced the photon absorption rate of the samples by as much as a factor of 2 in Ge:Be (728-8.7) and a factor of 8 in Ge:Ga (783-4.4). The lifetimes presented in Chapter 4 have been calculated with the assumption of a quantum efficiency of one; in this way the reported lifetimes provide a limit as to how small these values actually are, i.e., the actual lifetimes are equal to or greater than the calculated lifetimes.

\subsubsection{Aspects of the stressing configuration}

Beyond having an optical cavity and a built-in emitter, the main feature of the coldfinger is the capability of applying uniaxial compression to bar shaped samples. The uniaxial compression of a sample is achieved by turning the \#4-40 screw shown in Fig. 3.4 in the clockwise direction. The rounded tip of the screw exerts a force on the leaf spring. Because the leaf spring is supported by the stainless steel fulcrum, the force applied by the screw results in a force on the sample via the stainless steel piston. The mechanical analysis for the calculation of the stress applied is provided in Appendix A.

Although the actual stress on the sample does change due to the differences in the thermal expansion of the materials used (including 
stainless steel, brass, phosphor bronze, and the Ge sample), the stress is not expected to change very much in the narrow low temperature range in which the photo-Hall measurements are taken. In addition, as in the case of the stressed detectors, the leaf spring design should compensate for having a device constructed of materials with a variety of thermal expansion coefficients. A quantitative determination of this effect was not performed, but it was observed that at $15 \mathrm{~K}$ the change in the sample resistance between the fully unstressed case and that in which the piston was placed just in contact with the sample was slightly lowered. This suggests that the stress on the sample increased slightly when the coldfinger was cooled from $300 \mathrm{~K}$ to $15 \mathrm{~K}$.

\subsection{Materials selection and preparation}

Samples were produced from two Ga-doped Ge bulk crystals (773 and 783) and one Be-doped Ge bulk crystal (728). Approximately one millimeter thick slices were cut at a distance of $6.8 \mathrm{~mm}, 4.4 \mathrm{~mm}$ and 8.7 $\mathrm{mm}$ from the seed end of 773,783 and 728 , respectively. Samples are thus designated $773-6.8,783-4.4$, and 728-8.7. The sample configuration is depicted in Figure 3.3. The bars are $5 \mathrm{~mm}$ in length and have a $1 \times 1 \mathrm{~mm}^{2}$ cross section. All the surfaces of the samples are oriented in $\langle 100\rangle$ directions. In addition, 728-8.7 was annealed at $700{ }^{\circ} \mathrm{C}$ for 1 hour in a $\mathrm{KCN}$ soak prior to processing for electrical contacts. The anneal removes Be-H complexes, which form shallow levels in Ge, and the $\mathrm{KCN}$ (10 wt.\% in water) soak prevents copper from diffusing into the wafer during the annealing period. 
Electrical contacts consist of a layer with two B-implants $(25$ $\mathrm{keV} / 1 \times 10^{14} \mathrm{~cm}^{-2}$ and $\left.50 \mathrm{keV} / 2 \times 10^{14} \mathrm{~cm}^{-2}\right)$ and subsequent metallization. After the implantation procedure $20 \mathrm{~nm}$ of $\mathrm{Pd}$ and $800 \mathrm{~nm}$ of $\mathrm{Au}$ (in this sequence) were Ar sputtered on the implanted surfaces of the wafers. The slices were then annealed at $300{ }^{\circ} \mathrm{C}$ for 1 hour under Ar flow. This heat treatment removes implantation damage, activates the B implant (although up to $50 \%$ of the B may be electrically active as implanted in $\mathrm{Ge}$, and reduces stresses at the heterointerfaces. At this point $1 \times 1 \times 5 \mathrm{~mm}^{3}$ samples were diced from the wafers producing four bare and two fully metallized surfaces. All bare surfaces were then lapped in a water-alumina or water$\mathrm{SiC}$ slurry ( $\leq 1900$ grit). Three narrow lines (each about a half millimeter wide) of pirsne wax were painted on each of the metallized surfaces at locations designated for electrical contacts. Excess metal was then removed via a $\mathrm{KBr}$ etch $(\sim 1$ minute), and the exposed implanted layer was removed with a 3:1 $\mathrm{HNO}_{3}: \mathrm{HF}$ etch for 25 seconds. This final etching step also removes lapping damage from ali exposed surfaces. The picene wax was removed with a TCA rinse followed by a final methanol rinse.

Four of the six contäcts are necessary for making Hall measurements. The middle contact on either surface must be used. The other two may be configured in any manner so long as it is possible to inject current along the sample's length through the contacts chosen. Usually three copper wires (insulated) were connected on one side, and a fourth wire was connected to the middle contact on the other side. Wires were fixed to the metal pads with silver epoxy (cured at $125^{\circ} \mathrm{C}$ for 1 hour). 


\subsection{Sample mounting}

Samples were mounted with four objectives in mind: a) electrical insulation from the coldfinger, b) alignment with the piston, c) alignment with respect of the magnetic field, and d) effective heat-sinking. Samples were electrically insuiated from the coldfinger with thin pieces of mica $(\sim 25 \mu \mathrm{m})$ placed at each of the $1 \times 1 \mathrm{~mm}^{2}$ bare surface. Proper alignment with the piston was achieved by the use of a medium which served to compensate for any misalignment in the system (e.g. non-parallel sample surfaces, misaligned piston, etc.). Small pads cut out from an IBM computer card were used for most measurements. Some measurements on sample 728-8.7 were made with thin indium pads.

As can be noted from the Hall effect arrangement for the bar configuration, the $1 \times 5 \mathrm{~mm}^{2}$ contactless surfaces of the bar samples must be normal to the direction of the magnetic field. In order to accomplish this, the large faces of the coldfinger were chosen as a reference. Samples were aligned with respect to the coldfinger in this manner, and the coldfinger was then mounted onto the cryostat such that its faces (and therefore the contactless sample surfaces) were aligned normal to the field direction (Fig. 3.6). Finally, effective heat sinking was achieved with the use of GE varnish. The sample, the mica and the aligning pads were attached to the piston and the set screw via GE varnish. The varnish bridged the sample to the coldfinger reducing the reliance of heat sinking through the mica and pads. Miller-Stephenson 907 epoxy was also used as a heat sinking medium. Although more effective, the epoxy has a major drawback in that samples become fixed to the coldfinger beyond the point of their non- 
destructive removal. Sample mounting has become a continuously evolving aspect of this experiment.

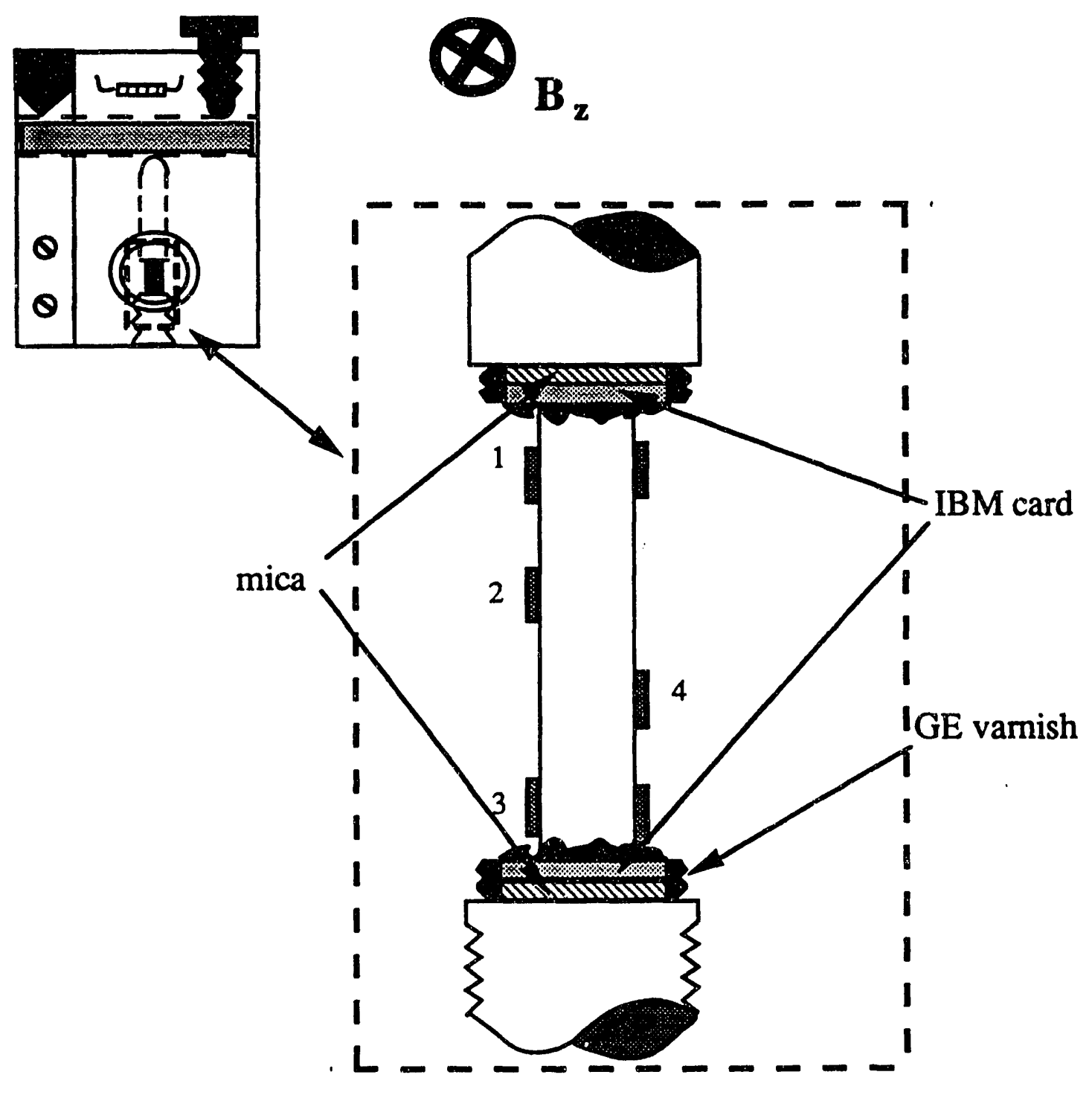

Figure 3.6. Schematic example of a mounted sample. 


\section{Results and discussion}

\subsection{Calibration results}

As mentioned in the introduction of this thesis, the work has been performed with two principal objectives in mind. The first objective has been to show that the photo-Hall technique can be used to measure the carrier lifetime in uniaxially stressed material. Chapter 3 describes the experimental methods allowing for the estimation of two of the parameters that most influence the success of this effort: the photon absorption rate and the stress applied. The best way to assess the degree of success achieved with these methods is to review the results of Hall and photo-Hall measurements.

\subsubsection{Photon absorption rate at $77 \mathrm{~K}$ and $99 \mathrm{~K}$}

Figure 4.1 is a plot of hole concentration for sample 783-4.4 as a function of temperature in the range where photon generation dominates. The data points above a concentration of $2 \times 10^{9} \mathrm{~cm}^{-3}$ correspond to photoexcitation by a $99 \mathrm{~K}$ source while the values below were recorded in the presence of a $77 \mathrm{~K}$ emitter. Because it is assumed that the spectral distribution of the emitter is that of a blackbody, the temperature determines the photon emission rate of the emitter. But not all photons incident on the sample are absorbed by the impurity centers in the material.

Figure 4.2 shows the spectral response of Ge:Ga material in the energy range where $\mathrm{Ga}$ impurities absorb photons. As photons are absorbed, the resistance of the sample decreases and is measured as the response of the material. One can see that photons of wavelengths between 


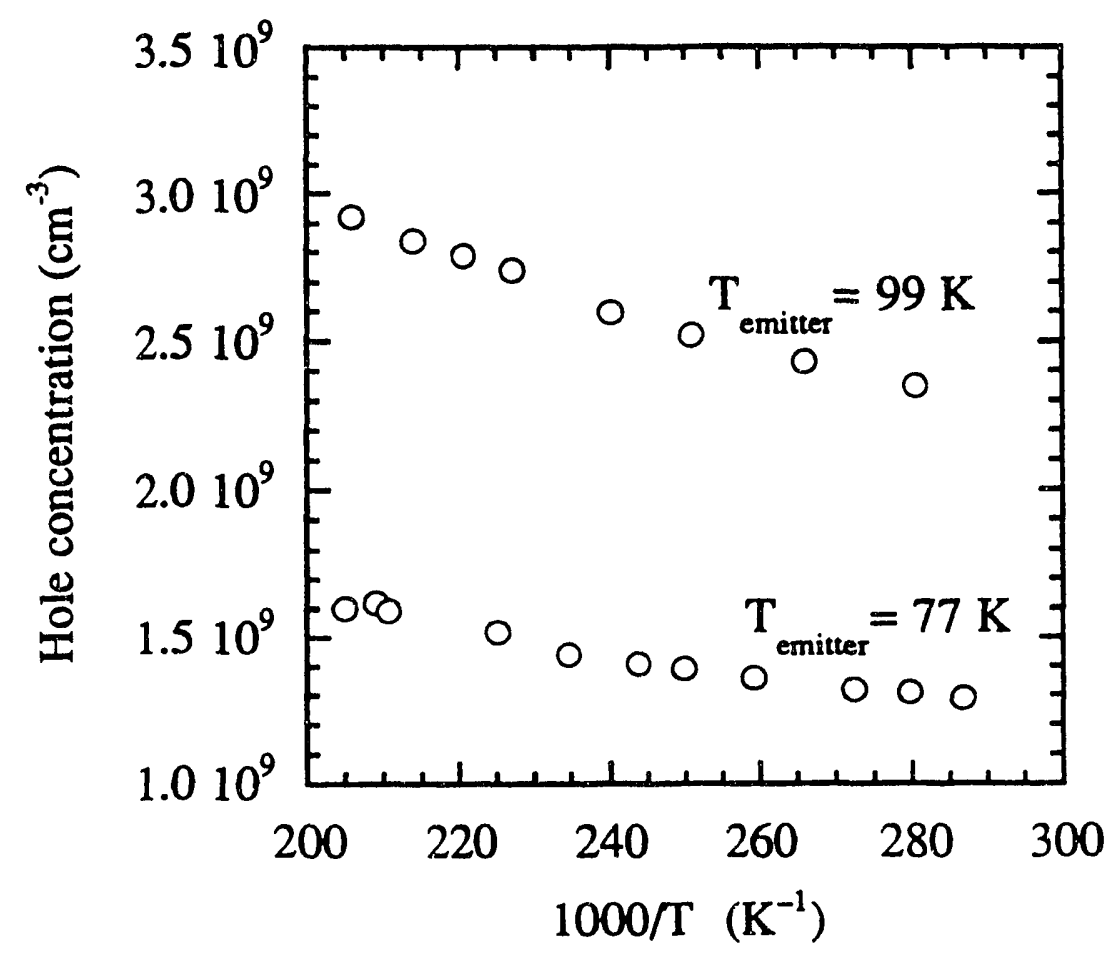

Figure 4.1. Concentration of photoexcited holes in sample 783-4.4. Two emitter temperatures were used in this case.

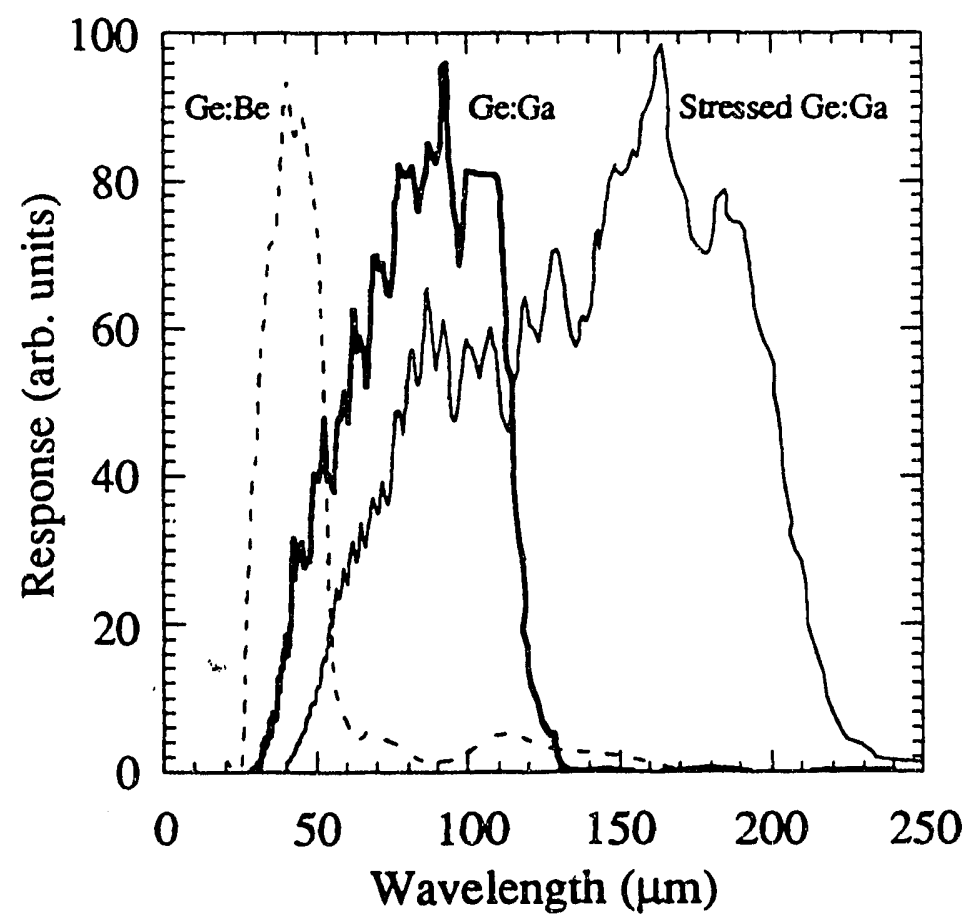

Figure 4.2. Photoconductive response as a function of photon wavelength. 
30 and 130 micrometers are absorbed. With this information it is possible to calculate the fraction of the blackbody spectrum that can be absorbed by the sample. Because the sample is in the cavity along with the emitter, the simplifying assumption is made that all photons emitted by the blackbody in the range of $30 \mu \mathrm{m}$ to $130 \mu \mathrm{m}$ are absorbed (Figure 4.3).

Numerical integration of the Planck blackbody distribution provides a quantitative result for the photon emission rate, which has been assumed to be equal to the rate of photon absorption by the sample. Therefore, for

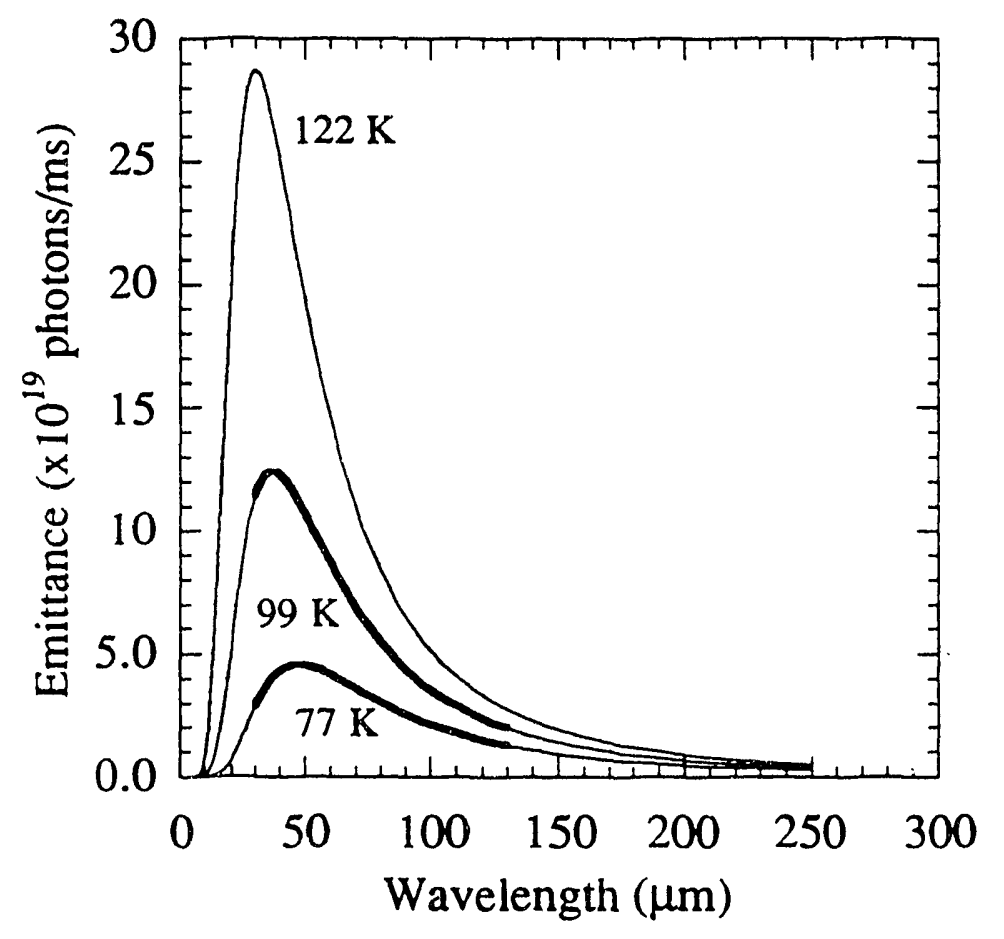

Figure 4.3. Blackbody spectra at $77 \mathrm{~K}, 99 \mathrm{~K}$ and $122 \mathrm{~K}$. The bold sections of the $77 \mathrm{~K}$ and $99 \mathrm{~K}$ spectra indicate the wavelengths of the photons that are absorbed by holes bound to Ga dopants in Ge:Ga. A larger number of photons is emitted by the $99 \mathrm{~K}$ blackbody. This produces a higher concentration of holes (Fig. 4.1). 
the $77 \mathrm{~K}$ emitter the photon absorption rate is about $3.0 \times 10^{15}$ photons/sec. In the case of the $99 \mathrm{~K}$ source the rate is approximately $6.4 \times 10^{15}$ photons/sec. Since the hole concentration measurements were performed on the same material, the ratio of the hole concentrations should equal that of the photon absorption rates in the low injection limit where the carrier lifetime is independent of photon flux. At $4 \mathrm{~K}$ the hole concentrations are $2.5 \times 10^{9} \mathrm{~cm}^{-3}$ and $1.4 \times 10^{9} \mathrm{~cm}^{-3}$ for the emitter temperatures of $99 \mathrm{~K}$ and $77 \mathrm{~K}$, respectively. Thus, the ratio of hole concentrations is 1.8 , and the ratio of emission rates (and absorption rates for the assumptions made) is 2.1 .

The implication of this result is that not all photons are absorbed. It is possible that a fraction of the shorter wavelength light is not absorbed because the ionization cross sections of $\mathrm{Ga}$ impurities at these wavelengths are small enough such that these photons may be absorbed elsewhere. So when the temperature of the emitter is changed and the peak of the blackbody distribution is shifted, the fraction of higher energy photons which may not be absorbed does not scale with the overall change in photon emission of the source (Fig. 4.3). Finally, this analysis provides no information regarding the absolute intensity of the emitter. That is, the validity of the assumption that the source is a perfect blackbody has not been tested.

\subsubsection{Stress calibration}

Appendix A provides a means of calculating the stress on the sample by mechanical analysis. The two variables which determine the stress being applied are the leaf-spring configuration and the degree to which the \#4-40 screw has been turned to deflect the leaf spring(s). The latter 
variable was eliminated by always turning the screw $1 / 4$ of a revolution. This is experimentally practical, for it facilitates the reproduction of measurements. Thus, the leaf-spring configuration is the determining factor.

The load applied to the sample is proportional to the product of the width $w$ and the cube of the height $t$ of the leaf spring. If more than one leaf spring is used, the total load is the sum of the load caused by each of the springs. The four arrangements used resulted in the following values of stress: $0.3 \mathrm{kbar}, 1.7 \mathrm{kbar}, 3.5 \mathrm{kbar}$, and $6.3 \mathrm{kbar}$. These calculated values can be compared to the actual stress applied by analysis of the binding energy of the acceptors associated with each state of stress. In his study of the strain dependence of binding energies of In- and Al-doped Ge, J.J. Hall ${ }^{9}$ provides an analytical formula describing the binding energies, $W$, as a function of strain, $\varepsilon$ :

$$
\mathrm{W}=\mathrm{W}_{\infty}+\mathrm{W}_{1} / \varepsilon+\mathrm{W}_{2} / \varepsilon^{2}
$$

where $W_{\infty}, W_{1}$, and $W_{2}$ are constants given by $4.75 \pm 0.15 \mathrm{meV}$, (11.2 \pm 1.5$) \times 10^{-3} \mathrm{meV}$, and $(-8.8 \pm 3.0) \times 10^{-6} \mathrm{meV}$, respectively, for the case of Ge:Al and compression in the [100] direction. These constants were determined from Hall effect measurements. Because the binding energy of $\mathrm{Al}(10.90 \mathrm{meV})$ is close to that of $\mathrm{Ga}(11.07 \mathrm{meV})$, this expression was used to compare the values for stress calculated following the method in Appendix A with the measured binding energies obtained from Hall measurements performed in this study. These results are discussed in the following section. 


\subsection{Experimental results in the Ge:Ga system}

4.2.1 Freeze out statistics where thermal generation dominates

Standard Hall effect measurements provide basic information concerning the majority and minority impurity concentrations in the samples. These are provided in Table 4.2.

\begin{tabular}{|c|c|c|}
\hline Sample I.D. & $\mathrm{N}_{\text {majority }}\left(\mathrm{cm}^{-3}\right)$ & $\mathrm{N}_{\text {minority }}\left(\mathrm{cm}^{-3}\right)$ \\
\hline $773-6.8$ & $1.4 \times 10^{14}$ & $1.4 \times 10^{12}$ \\
\hline $783-4.4$ & $1.0 \times 10^{14}$ & $5 \times 10^{12}$ \\
\hline $728-8.7$ & $1.2 \times 10^{15}$ & $2.4 \times 10^{12}$ \\
\hline
\end{tabular}

Table 4.2. Impurity concentrations for the samples tested.

These values were obtained by fitting the Hall data with the semiconductor statistics discussed in Chapter 2. It should be noted that the actual statistics involved in carrier freeze-out of double acceptors is somewhat more complicated than the simple expressions governing simple impurity centers.

For samples under stress, Hall measurements yield information regarding the binding energy of the acceptors. Given the information in Table 4.2, the binding energies of $\mathrm{Ga}$ centers in $783-4.4$ are $6.7 \mathrm{meV}$ and $7.3 \mathrm{meV}$ at the calculated compressions of $6.3 \mathrm{kbar}$ and $3.5 \mathrm{kbar}$, respectively. The binding energies corresponding to $6.3 \mathrm{kbar}$ and $3.5 \mathrm{kbar}$ are $6.4 \mathrm{meV}$ and $7.3 \mathrm{meV}$, respectively, when computed from Equation 4.1 (given a Young's modulus of $1.06 \times 10^{3} \mathrm{kbar}$ ). It should be noted that subsequent to the Hall measurements of 783-4.4 performed at $3.5 \mathrm{kbar}$ and 
$6.3 \mathrm{kbar}$, a run of the same sample was made for a calculated stress of 1.7 $\mathrm{kbar}$, and the results matched the data taken a $3.5 \mathrm{kbar}$.

In addition to the impurity concentrations listed above, it was necessary to account for the effect of stress on the effective density of states of the valence band in the fitting of the freeze out curves. This was achieved by changing $B$ from 4 to 2 since the band edge goes from having a spin degeneracy factor of four to two and by exchanging the density of states effective mass from $0.37 \mathrm{~m}_{e}$ to $0.082 \mathrm{~m}_{e}$ when going from the unstressed to the stressed condition. This new effective mass--which is equal to the cube root of the product of the longitudinal mass and the square of the transverse effective mass--is that of the deformed "heavy" hole band. 39,40

Figure 4.4 elucidates many of the points just discussed. Freeze-out curves of 783-4.4 are shown for the two states of stress described above (6.3 kbar and $3.5 \mathrm{kbar}$ ) and for an unstressed sample with data taken using the van der Pauw configuration. The solid lines matching the data sets are the theoretical fits, each with the appropriate effective mass and spin degeneracy factor. The dashed line that does not match any data set is the theoretical curve for an unstressed sample having impurity concentrations equal to those in 783-4.4 but with an impurity binding energy of $6.7 \mathrm{meV}$, (i.e., like that of the $6.3 \mathrm{kbar}$ measurement).

\subsubsection{Carrier lifetime}

The change in the hole concentration between stressed and unstressed states in the temperature range of optical generation dominance reflects a change in the carrier generation rate and lifetime (Fig. 4.5). The hole generation rate is equal to the product of the photon absorption rate and the 


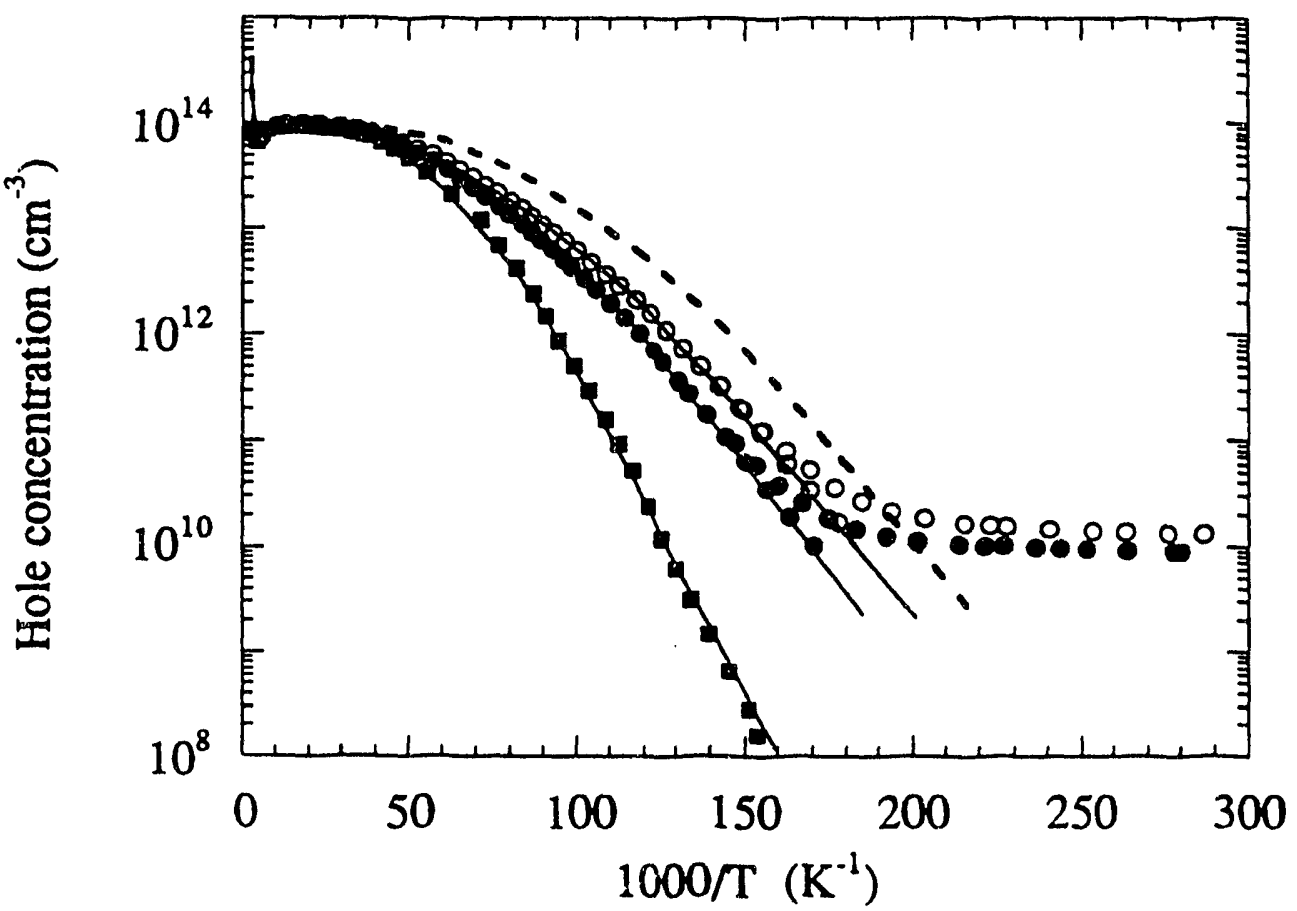

Figure 4.4. Freeze-out curves for sample 783-4.4 for three states of stress: unstressed (filled squares), $3.5 \mathrm{kbar}$ (filled circles), and $6.3 \mathrm{kbar}$ (clear circles). The lines are theoretical fits which were obtained as described in the text.

sample volume ( $5 \times 10^{-3} \mathrm{~cm}^{3}$ in the present case) if photon absorption occurs uniformly throughout the sample. If a $77 \mathrm{~K}$ blackbody is considered, the hole generation rate in a Ga-doped Ge sample is $6 \times 10^{17} \mathrm{~cm}^{-3} \mathrm{~s}^{-1}$. For the samples under stress, the photon absorption rate changes because the impurity centers can absorb photons of lower energy. The extra photons are associated with the tail of the Planck distribution (i.e., long wavelengths) and consequently are relatively few in number. The resulting absorption rate increases by about $10 \%$ to $3.3 \times 10^{15}$ photons/sec which translates into a carrier generation rate of $6.6 \times 10^{17} \mathrm{~cm}^{-3} \mathrm{~s}^{-1}$. The resulting calculated lifetimes at $4 \mathrm{~K}$ for 783-4.4 are given in Table 4.3. In the high stress limit the calculated lifetime increases by a factor of 10 . 


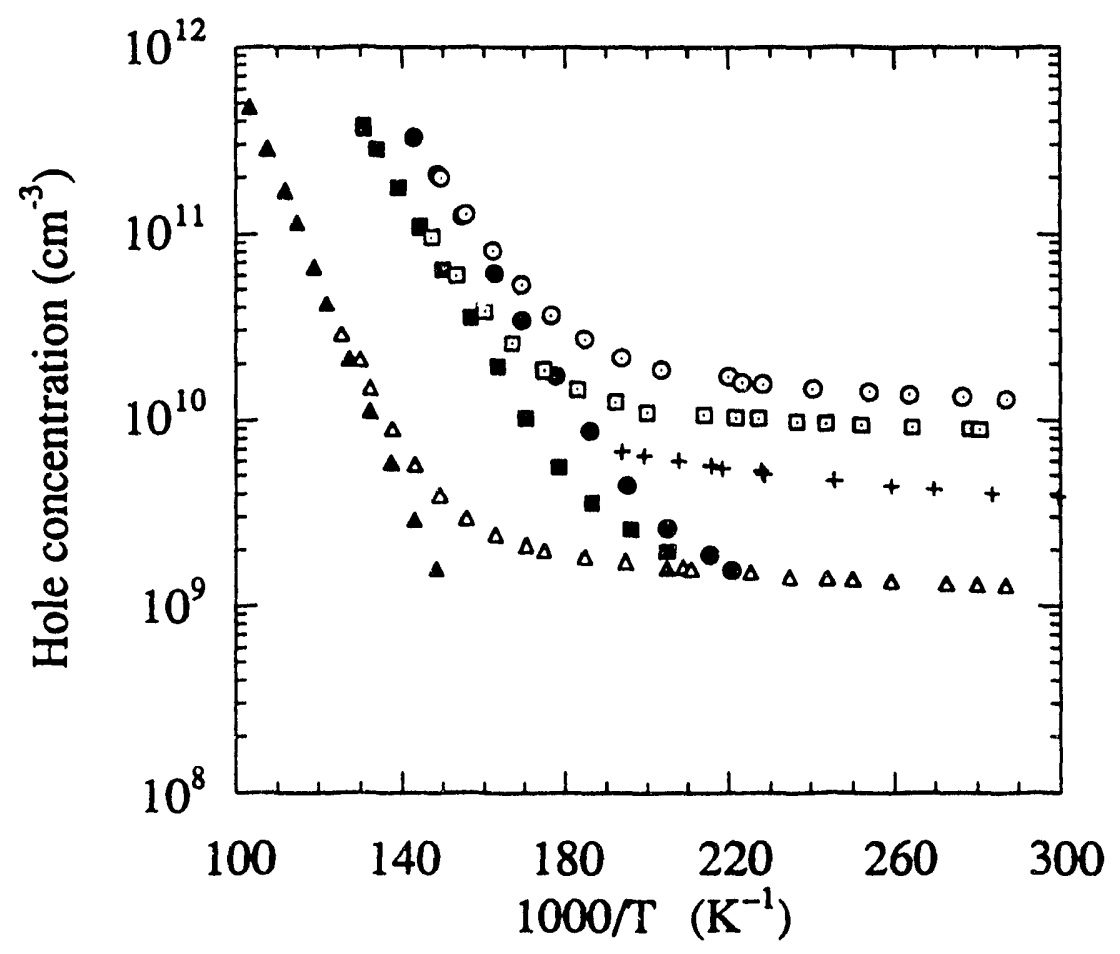

Figure 4.5. Hole concentrations in sample 783-4.4 under three states of stress: $6.3 \mathrm{kbar}$ (circles), $3.5 \mathrm{kbar}$ (squares), and unstressed (triangles). The filled symbols refer to dark Hall and clear symbols to photo-Hall measurements. The cross legend $(+)$ denotes photo-Hall data for unstressed 773-6.8. In the nearly flat regions below $5 \mathrm{~K}(1000 / \mathrm{T}=200)$, holes were generated optically, and the temperature dependence of the hole lifetime is that of the hole concentration.

\begin{tabular}{|c|c|c|}
\hline Stress (kbar) & Generation $\left(\mathrm{cm}^{-3} \mathrm{~s}^{-1}\right)$ & Lifetime $(\mathrm{s})$ \\
\hline 0 & $6.0 \times 10^{17}$ & $2.3 \times 10^{-9}$ \\
\hline 3.5 & $6.6 \times 10^{17}$ & $1.4 \times 10^{-8}$ \\
\hline 6.3 & $6.6 \times 10^{17}$ & $2.2 \times 10^{-8}$ \\
\hline
\end{tabular}

Table 4.3. Calculated lifetimes for sample $783-4.4$ at $4 \mathrm{~K}$ for the three states of stress indicated in the first column. 
Because lifetime is inversely proportional to the concentration of capture centers, the lifetimes listed in Table 4.3 are specific to the sample. For the temperatures at which the photo-Hall measurements were performed, the concentration of capture centers corresponds to the minority impurity concentration. Thus, Fig. 4.5 shows that the hole concentration in the region of optical generation is higher for 773-6.8 than for 783-4.4 approximately by a factor corresponding to the ratio of the inverses of the minority impurities $(\sim 3)$. Because both measurements are for Ga-doped, unstressed samples in the presence of a $77 \mathrm{~K}$ blackbody, the difference in the hole concentrations gives directly the change in the lifetimes.

Kaufman et al.54 have performed lifetime measurements in p-Ge (with shallow acceptors) by the generation-recombination noise technique revealing a power law dependence of $T^{n}$ where $n$ is between 2.4 and 3 . This is in good agreement with the model for the capture cross section proposed by Abakumov et al. in which the capture cross section is proportional to $T^{-3}$ (assuming the mean velocity is proportional to $T^{1 / 2}$ ). Clearly the data for 783 and 773 (Ga-doped crystals) do not exhibit such a strong temperature dependence. Because the temperature range over which hole lifetimes were extracted is so small, a dependence could not be determined precisely. However, to first order the hole concentrations (and therefore the ifetimes) are proportional to $T^{n}$ where $n=1.0 \pm 0.3$.

The discrepancy between these results and the data of Kaufman et al. is at the present time not well understood. One possibility is that a significant fraction of the optically generated carriers is "hot"; that is, the holes which have been excited to the valence band by the blackbody radiation behave as if having a mean energy higher than that which would 
correspond with a normal thermal distribution of carriers at the lattice (sample) temperature. Even as the lattice is cooled, the energy of the holes need not be correspondingly reduced, i.e., the holes may be considered thermally decoupled from the lattice. Carriers can become hot with the application of high electric fields. But higher energy carriers can also be produced when high energy photons are used in the excitation process. Kaufman et al. provide evidence indicating that photons of energies smaller than $25 \mathrm{meV}$ will not produce hot carriers in Ge doped with shallow impurities having minority impurity concentrations comparable to those of 773-6.8 and 783-4.4. If the lifetime of the holes is of the order of the mean time between energy relaxation events (usually meaning relaxation time for lattice scattering events), the holes will not thermalize prior to being captured. In addition, other effects such as free carrier absorption may prevent the holes in the valence band from achieving a thermal distribution. Measurements in which greater than $25 \mathrm{meV}$ photons were partially filtered have not provided clarification at the present time.

\subsubsection{Carrier mobilities}

As mentioned in Sec. 2.8, the effect of uniaxial stress on the Hall mobility of holes in Ge doped with shallow acceptors has been well documented. The work in the early sixties by John J. Hall ${ }^{9}$ remains the fundamental experimental evidence showing the factor of two increase of the Hall mobility in the high stress limit for bar-shaped samples (where the current was injected in a direction parallel to the direction of compression). J.J. Hall proposed that the change in the mobility with stress could be due to the change in the hole effective mass but did not provide a detailed analysis on account that the mobility may depend on a combination 
of both ionized and neutral impurity scattering at low temperatures (6.2 $\mathrm{K})$, each scattering mechanism having a different effective mass dependence. The impact of changes in the effective mass on the mobility of Ge under stress has also been supported by recent experiments. 57

Figure 4.6 is a plot of the Hall mobility as a function of inverse temperature for sample 783-4.4. The data depict measurements performed under no stress, $3.5 \mathrm{kbar}$, and $6.3 \mathrm{kbar}$. The filled symbols refer to Hall measurements made in the absence of light while the open symbols correspond to photo-Hail experiments. A.t $6.2 \mathrm{~K}$ the dark Hall data indicate an increase of a factor of 2 in the mobility between the unstressed and the stressed conditions in good agreement with J.J. Hall's observations. In addition, these data show a maximum in the mobility (as a function of temperature) and a decrease at the lower temperatures regardless of the state of stress. This general sh`pe reveals that ionized (and perhaps neutral) impurity scattering exists in the temperature range where the majority of free carriers are optically generated.

From the photo-Hall results in Fig. 4.6, one can see that the optically generatea holes are of higher mobility than those excited thermally into the valence band. This effect is observed in the three states of stress that were investigated. These photo-Hall mobilities appear to be very weakly dependent on temperature. Such behavior suggests once more that hot carriers are being created optically. The temperature dependence for ionized impurity scatiering arises from the assumption that the mean energy of carriers in thermal equilibrium is proportional to $k_{B} T$ where $T$ is the lattice temperature. But the energy distribution of the holes is 


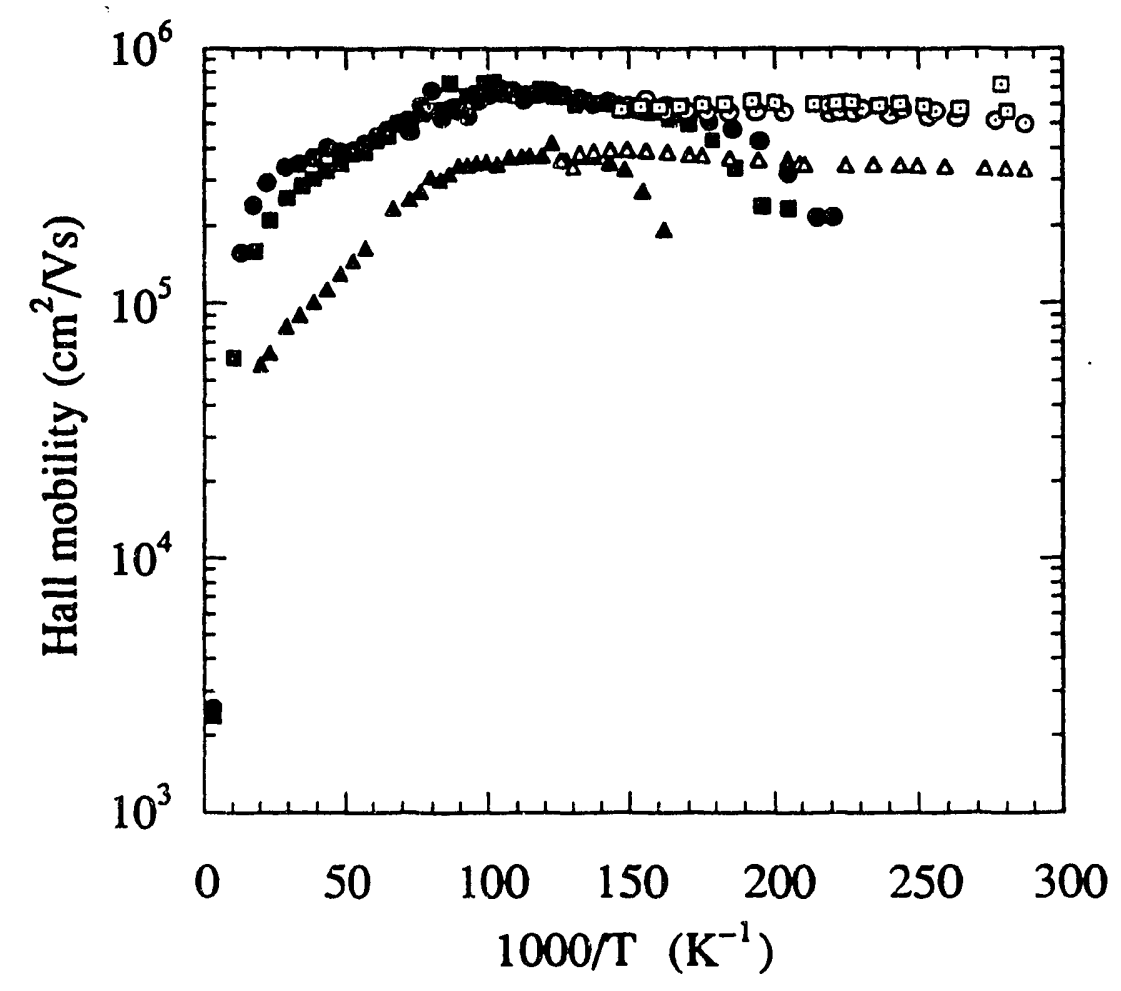

Figure 4.6. Hall mobility corresponding to the hole concentration data in Fig. 4.5. The same convention has been used in both figures. 
associated with the position in the valence band to which they are optically excited and their ability to interact with the lattice in energy/momentum losing processes. Under a temperature independent optical generation rate (assumed to be the case in this study) the mean energy of the hot holes is then weakly dependent on the lattice temperature, and the mobility should achieve a roughly constant value. One should not attribute this behavior exclusively to hot holes. Further experiments are necessary.

\subsection{Experimental results in the Ge:Be system}

\subsubsection{Free carrier statistics and lifetimes in $\mathrm{Ge}: \mathrm{Be}$}

Beryllium doped germanium samples were studied in a similar manner to that described for Ge:Ga crystals. Results were obtained for several states of compressive stress: unstressed, $0.3 \mathrm{kbar}, 1.7 \mathrm{kbar}, 3.5$ kbar, and 6.3 kbar. Fig. 4.7 is a plot of the hole concentration as a function of inverse temperature for 728-8.7\#2. (Because several bar samples were cut from slice $728-8.7$, additional notation has been used to differentiate among bars \#1, \#2, \#3, etc.) The beryllium and mirority (donor) impurity concentrations are approximately $1 \times 10^{15} \mathrm{~cm}^{-3}$ and $2.4 \times 10^{12} \mathrm{~cm}^{-3}$, respectively. As was observed in the Ge:Ga system, the application of uniaxial compression reduces the amount of energy required to excite a hole from the a neutral $\mathrm{Be}$ atom; thus, the carrier concentration at any given temperature in the freeze-out region increases with the applied stress. Below $10 \mathrm{~K}$ the measured hole concentration is largely due to photoexcitation of neutral Be. From this data one can immediately see that in the low temperature limit the hole concentration increases by more than 


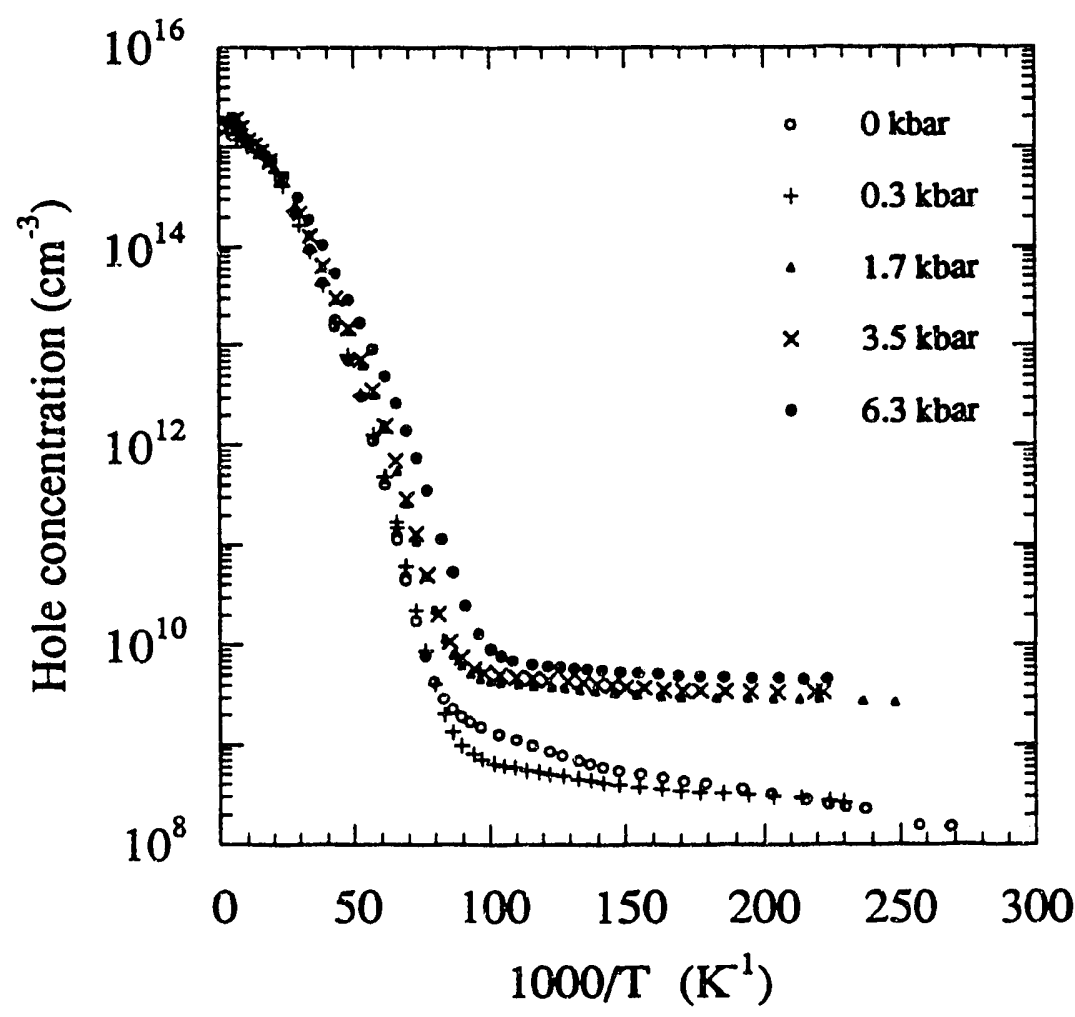

Figure 4.7. Hole concentration versus inverse temperature for sample 7288.7\#2. Each type of legend corresponds to a different state of stress as labeled in the figure. The nearly flat regions below $10 \mathrm{~K}(1000 / \mathrm{T}=100)$ correspond to photo-Hall measurements where photoexcitation dominated.

one order of magnitude with stress indicating a significant increase in the hole lifetime.

The energy associated with the first ionization state of $\mathrm{Be}$ is more than two times greater than that of $\mathrm{Ga}$ in $\mathrm{Ge}$. Consequently, the onset of carrier freeze-out occurs at a higher temperature, and optical generation of holes becomes significant at a higher temperature as well. It is possible then to obtain with the available experimental apparatus more precise information regarding the temperature dependence of the hole lifetime in the Ge:Be system than in the Ge:Ga system. In addition, the spectral response for $\mathrm{Be}$ centers is different than for $\mathrm{Ga}$ impurities requiring the 
recalculation of the rate of photon absorption. For unstressed samples the estimated photon absorption rates are $9.6 \times 10^{14}$ photons/s and $6.5 \times 10^{15}$ photons/s for emitter temperatures of $77 \mathrm{~K}$ and $122 \mathrm{~K}$, respectively. These yield generation rates of $4.8 \times 10^{17} \mathrm{~cm}^{-3} / \mathrm{s}$ and $3.2 \times 10^{18} \mathrm{~cm}^{-3} / \mathrm{s}$, respectively. Such values increase by approximately $20 \%$ for the cases in which stress $>1.5 \mathrm{kbar}$ has been applied (due to a reduction in the ionization energy). The change in the carrier concentration with emitter temperature is well illustrated in Figure 4.8a (sample 728-8.7\#4). Hole lifetimes calculated for temperatures below $10 \mathrm{~K}$ are presented in Fig. $4.8 \mathrm{~b}$.

Because hole concentrations slight electric field dependence, lifetimes were obtained for holes which were photoexcited by the $122 \mathrm{~K}$ emitter. A higher generation rate decreases the resistivity of the sample reducing the electric field in the sample for a given applied current (equal to $20 \mathrm{nA}$ for most of the photoHall data). Figure $4.8 \mathrm{~b}$ reveals a reduction in the temperature dependence of the calculated hole lifetimes and a significant enhancement of the lifetime (by as much as a factor of 20 at $5 \mathrm{~K}$ ) as the stress is increased. In fact, extrapolation of the data down to $3 \mathrm{~K}$ suggests that the lifetime of holes even for a stress of $0.3 \mathrm{kbar}$ is a factor of two greater than the lifetime when no stress is applied. Consequently, above $5 \mathrm{~K}$ the holes in the lightly stressed material have a shorter lifetime than in the unstressed material, but the opposite is true at lower temperatures. Evidence of this effect is given in Figure 4.9, a plot of hole concentration versus temperature for sample $728-8.7 \# 3$ measured in the presence of a $77 \mathrm{~K}$ emitter. (The hole concentrations are proportional to the lifetime when the generation rate is constant.) It is clear that the capture dynamics in the Ge:Be system is very sensitive to compression. 


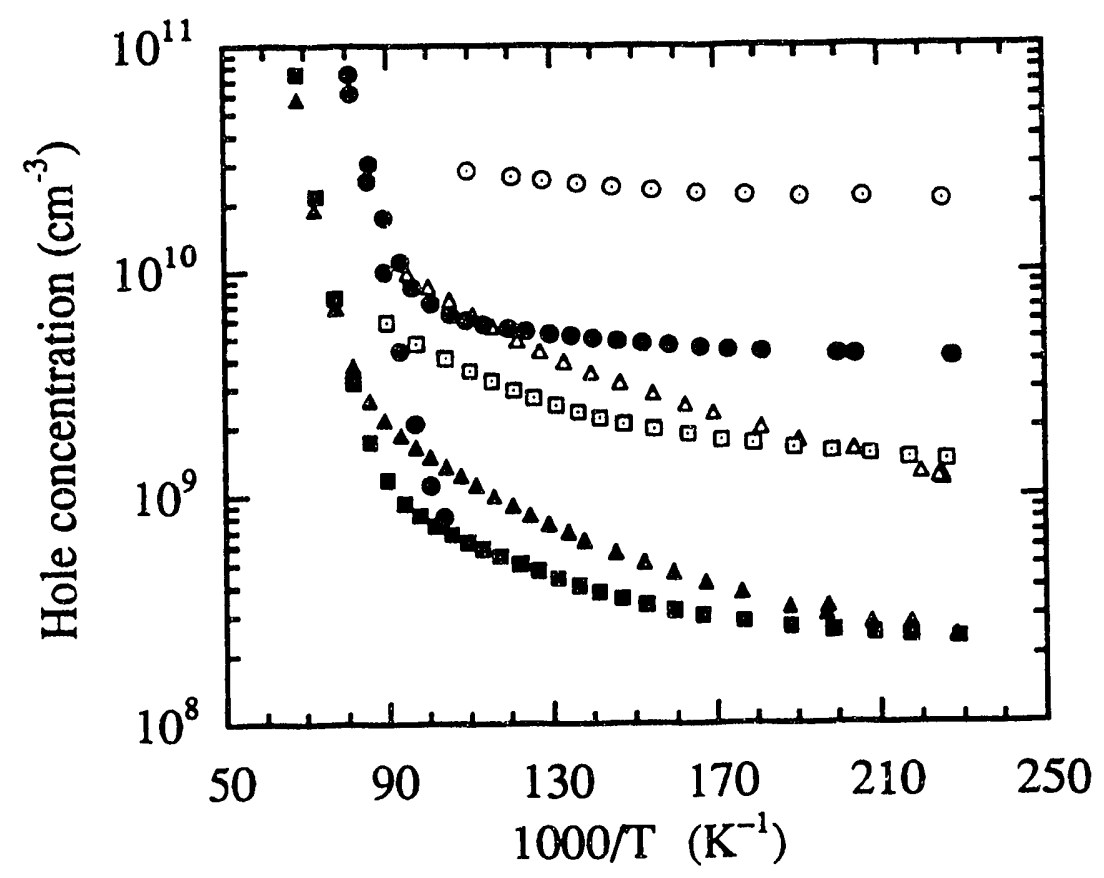

(a)

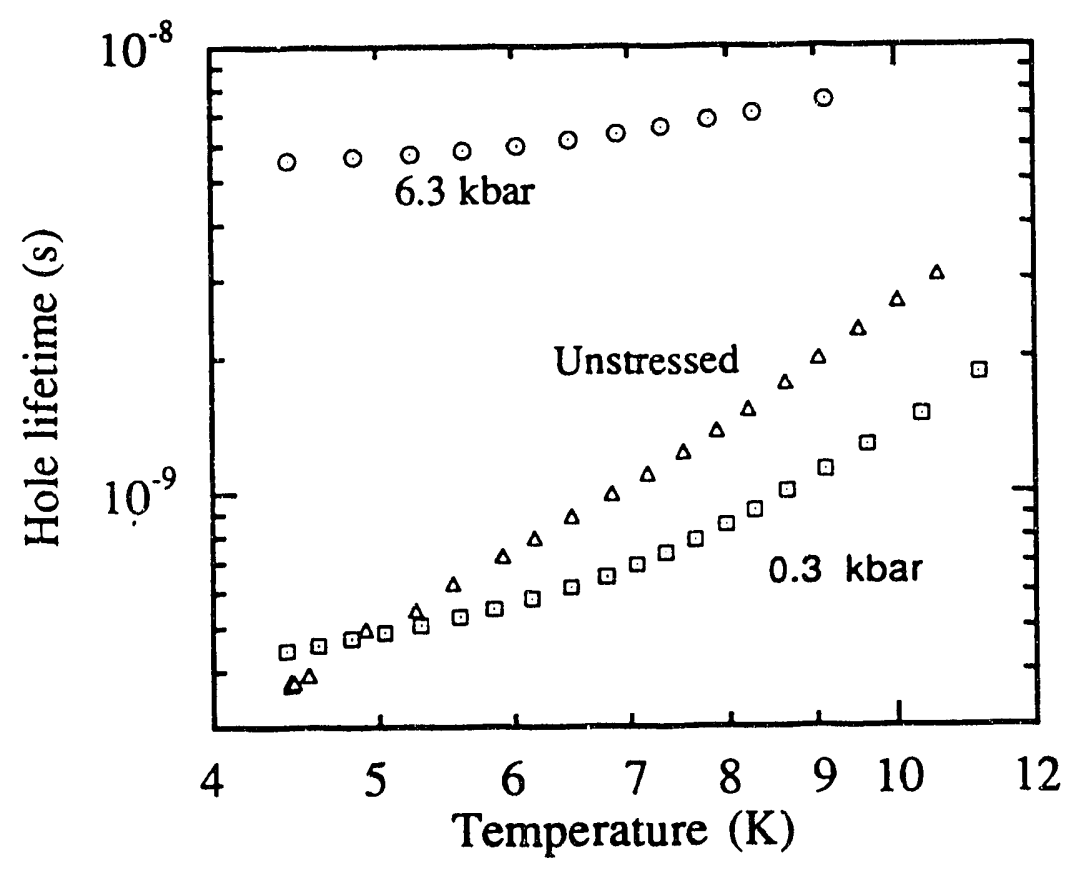

(b)

Figure 4.8. (a) Concentration of photoexcited holes in sample 728-8.7\#4. The filled symbols correspond to exictation by a $77 \mathrm{~K}$ emitter while the clear were obtained with a $122 \mathrm{~K}$ emitter. Symbols correspond to different states of stress: $6.3 \mathrm{kbar}$ (circles), $0.3 \mathrm{kbar}$ (squares) and unstressed (triangles). (b) Lifetimes computed from the data measured with the 122 $\mathrm{K}$ emitter. Symbols are consistent with (a). 


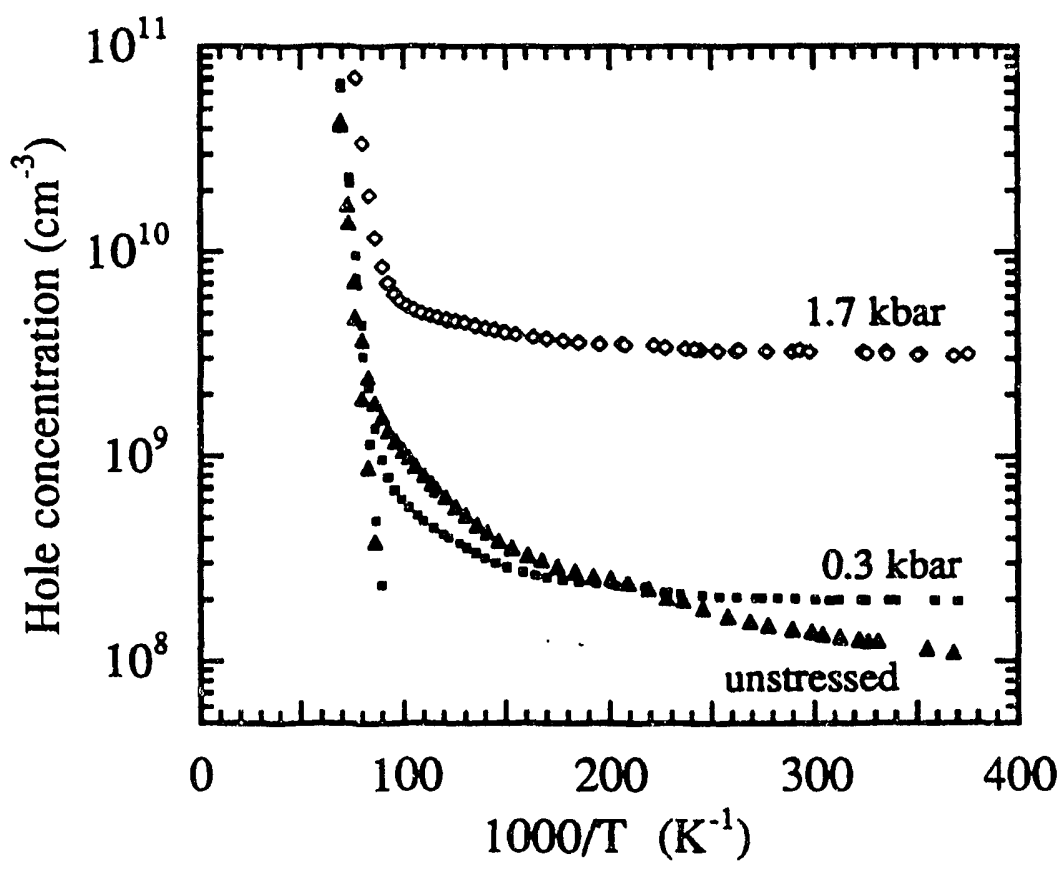

Figure 4.9. Hole concentration in sample $728-8.7 \# 3$ for three states of stress. Above $5 \mathrm{~K}(1000 / \mathrm{T}=200)$ the hole concentration of photoexcited holes in the unstressed case is higher than the concetration when $0.3 \mathrm{kbar}$ is applied. However, the opposite is true below $5 \mathrm{~K}$ indicating a reduction in the temperature dependence of the hole concentration as stress is applied. At the lowest temperatures measured a stress of $1.7 \mathrm{kbar}$ increases the hole concentration by more than one order of magnitude. This suggests that the hole lifetime increases strongly as well.

The origin of this effect has not yet been deciphered. However, the first step toward addressing the problem is to consider the materials aspects that change upon the application of a small amount of stress. The fourfold degeneracy of both the excited energy states and the valence band edge is lifted when uniaxial compression is applied (Sec.2.8). Such changes result in a reduction of the ionization energy of shallow levels. In addition, the binding energy of the third hole of $\mathrm{Be}^{+}$is rapidly lowered.18 Sufficient splitting of these levels eliminates the presence of $\mathrm{Be}^{+}$centers thus allowing 
this overcharged state to exist only for compressions below $0.8 \mathrm{kbar}$. So, for a given temperature the concentration of $\mathrm{Be}^{+}$centers decreases as the pressure is increased up to $0.8 \mathrm{kbar}$. In the low stress limit the curvatures and consenquently the effective masses for the light and heavy hole bands do not change very much. The relative shifts of the bands should not significantly alter their relative carrier population since about $94 \%$ of the free holes already occupy the heavy hole band when zero stress is applied.

With these points in mind, is it possible to provide a qualitative descripition of the effect of uniaxial compression in the Ge:Be system? One can only propose some possibilities. The stability of $\mathrm{Be}^{+}$is very sensitive to stress making the overcharged centers potentially significant contributors to the dynamics of hole caputre. Analysis of transient response measurements by Haegel et al. 58 have indicated that trapping by neutral $\mathrm{Be}$ (forming $\mathrm{Be}^{+}$) in samples with a Be concentration less than 1014 $\mathrm{cm}^{-3}$ does not affect the steady state concentration of photoexcited holes under low level excitation conditions at the temperatures where thermal generation is negligible. Under these circumstances the concentration of negatively charged beryllium is approximately equal to the concentration of compensating minority impurities. This leads to the rate equations

$$
\frac{\mathrm{dp}}{\mathrm{dt}}=\mathrm{g}-\frac{\mathrm{p}}{\tau_{\mathrm{p}}}-\mathrm{B}_{\mathrm{Be}}{ }^{\mathrm{p}} \mathrm{N}_{\mathrm{Be}}{ }^{\circ}+\mathrm{B}_{\mathrm{Be}}{ }^{\circ} \mathrm{N}_{\mathrm{Be}}{ }^{+} \mathrm{N}_{\mathrm{v}} \exp \left(-\frac{E}{\mathrm{k}_{\mathrm{B}} \mathrm{T}}\right)
$$

and

$$
\frac{\mathrm{d}\left(\mathrm{p}+\mathrm{N}_{\mathrm{Be}}{ }^{+}\right)}{\mathrm{dt}}=\mathrm{g}-\frac{\mathrm{p}}{\tau_{\mathrm{p}}},
$$


where $\mathrm{BBe}^{\mathrm{O}}$ is the recombination conefficient, $\mathrm{E}$ is the binding energy of the third hole, and all other terms are as previously defined.

If the neutral and overcharged $\mathrm{Be}$ centers are assumed to be in thermal equilibrium with each other, one may approximate the $\mathrm{Be}^{+}$ concentration under steady state conditions:

$$
\mathrm{N}_{\mathrm{Be}}{ }^{+}=\frac{\mathrm{pN}_{\mathrm{Be}}}{\mathrm{N}_{\mathrm{v}} \exp \left(-\frac{E}{\mathrm{k}_{\mathrm{B}} \mathrm{T}}\right)} .
$$

It is then possible to calculate the concentration of $\mathrm{Be}^{+}$centers as a function of temperature for the cases of zero stress and $0.3 \mathrm{kbar}$ under the illumination conditions which have already been described. The binding energies $E$ were estimated as $4.5 \mathrm{meV}$ and $4.0 \mathrm{meV}$ for zero stress and 0.3 kbar, respectively. Figure 4.10 is a plot of the hole and $\mathrm{Be}^{+}$concentrations as functions of temperature. As the sample is cooled, the concentration of $\mathrm{Be}^{+}$centers increases becoming comparable to the minority carrier concentration. Clearly, the experimental work presented here does not meet the condition of low level photoexcitation, for in the low temperature limit the estimated values of $\mathrm{NBe}^{+}$violate the starting assumption that $\mathrm{N}_{\mathrm{D}}>>\mathrm{NBe}^{+}$. This does not necessarily mean that $\mathrm{Be}^{+}$formation plays a dominant role in the trapping of holes but does mean that trapping by neutral Be must be considered.

If the formation of $\mathrm{Be}^{+}$centers is a strong factor in determining the hole lifetimes, one can view the measured lifetimes to be composed of several processs, two of which are depicted in Fig. 4.11. Holes can be optically excited from neutral centers at a rate that is independent of 


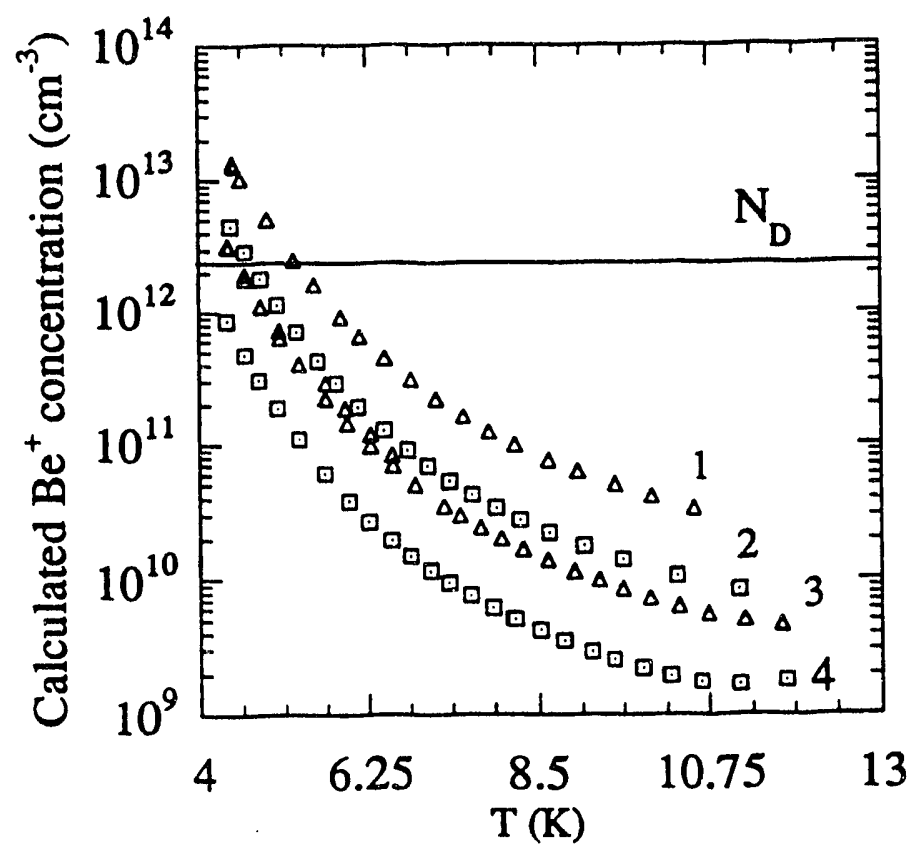

Figure 4.10. Calculated concentration of $\mathrm{Be}^{+}$centers in photon background conditions at low temperatures. The triangles correspond to unstressed Ge and squares to $0.3 \mathrm{kbar}$. 1 and 2 are for the case of $122 \mathrm{~K}$ emitter and 3 and 4 to $77 \mathrm{~K}$ emitter.

termperature. They are thermally excited from the overcharged sites at a rate that does depend on temperature. The recombination process can occur at $\mathrm{Be}^{-}$or $\mathrm{Be}^{\circ}$ sites, each type having associated with it a range of lifetimes which depends on the energy distribution of free holes. Thus, the measured lifetime in the low temperature region can be a combination of a variety of capture processes. A broad band source such as the one used in this study may also result in the photoexcitation of $\mathrm{Be}^{+}$centers. One may also have to account for trapping by residual shallow acceptors (which are assumed to exist in very low quantities in these samples). 


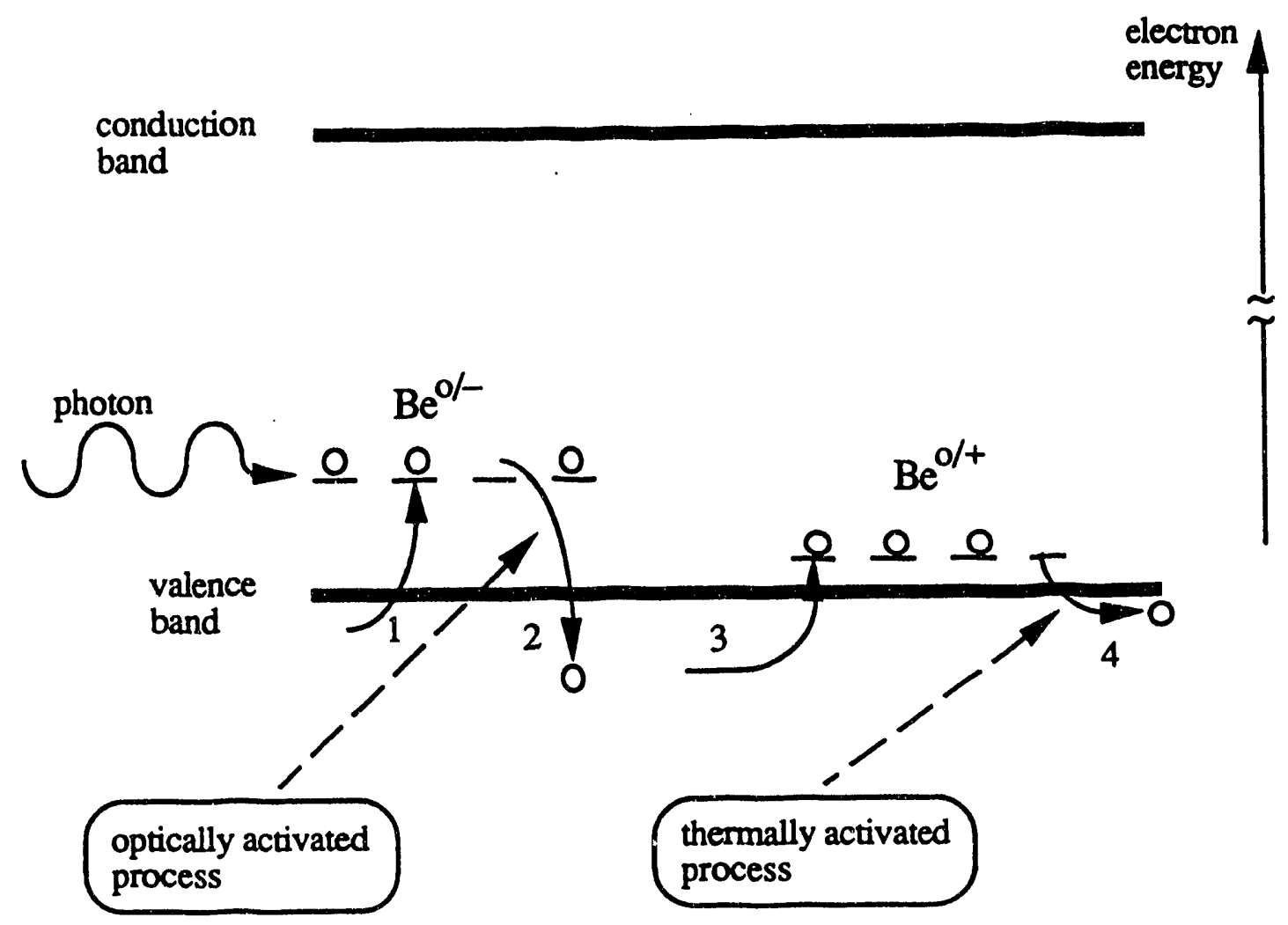

Figure 4.11. Generation-recombination (G-R) processes that are possible if $\mathrm{Be}^{+}$centers play an important role. G-R permutations of processes $1-4$ provide the possibilities.

\subsubsection{Hall mobilities in the Ge:Be system}

Hall mobilities have been calculated for the data appearing in Figure 4.8 and are plotted as a function of inverse temperature in Figure 4.12. The actual values are not as important as the general features which change with stress and temperature. Each set of data exhibits distinct regions in 


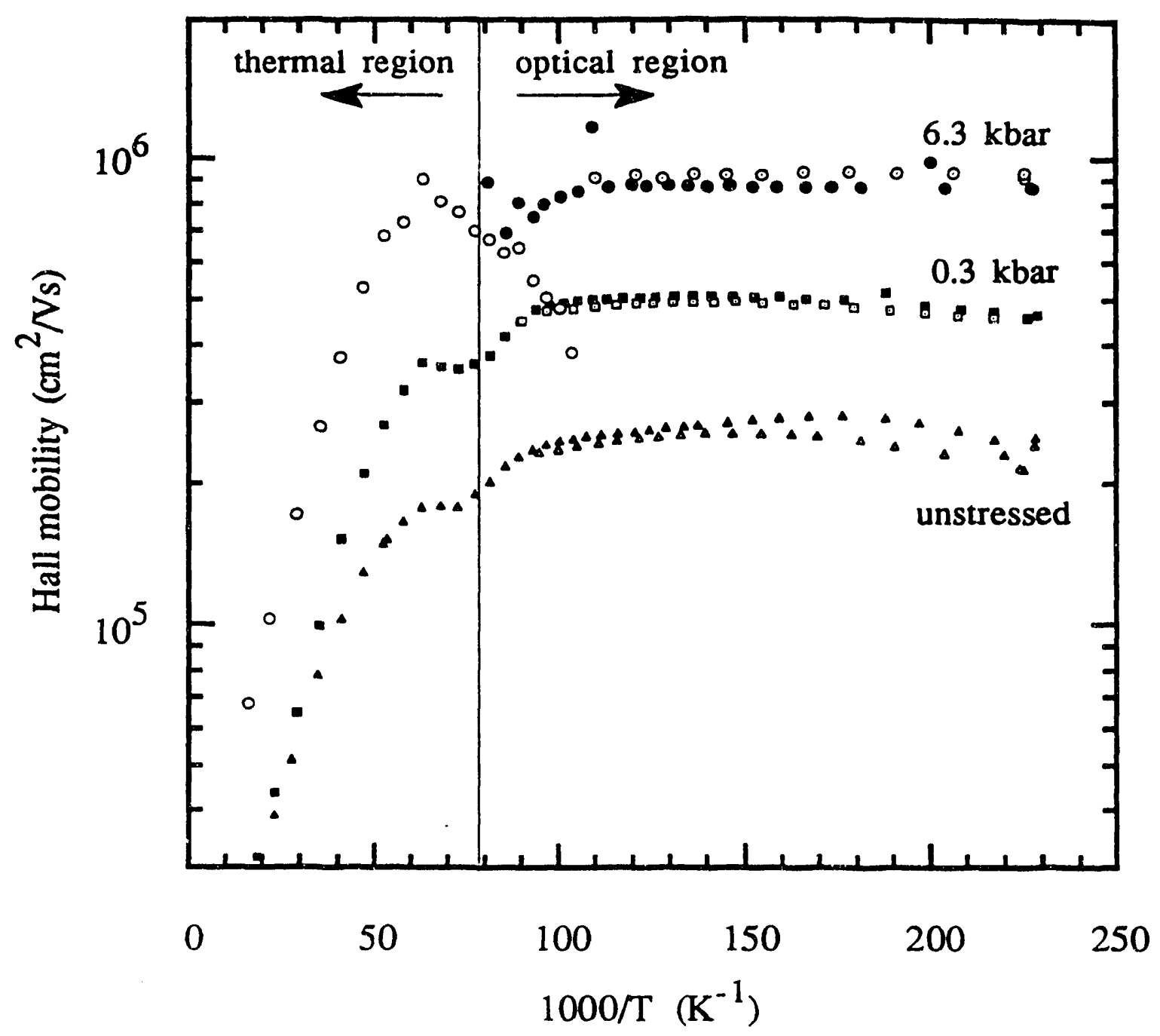

Figure 4.12. Hall mobility of holes in sample $728-8.7 \# 4$ for three states of stress as indicated in the plot. In the optical region, hole generation is dominated by photoexcitation (except for the dark Hall measurements performed under a stress of $6.3 \mathrm{kbar}$ and indicated in the plot by the clear circles). The filled symbols in the optical region represent data taken in the presence of a $77 \mathrm{~K}$ emitter. The clear or dotted symbols represent data taken in the presence of a $122 \mathrm{~K}$ emitter. 
which either the thermal or the optical generation of hole appears to dominate. The temperature at which the transition between these regions occurs is approximately $13 \mathrm{~K}$ for all states of stress with photoexcitation predominating in the lower temperature range. Above $13 \mathrm{~K}$ the major feature common to the three states of stress corresponds to the transition from lattice scattering to ionized/neutral impurity scattering. Such a transition appears as a maximum in the mobility and is best shown by the data depicted with open circles (Fig. 4.12). The region dominated by optical excitation is characterized by an abrupt increase in the mobility with a subsequent reduction in its temperature dependence. These features have also been observed 59 and analyzed 59,60 in the $\mathrm{Ge}: \mathrm{Cu}$ system. The authors attribute the abrupt increase in mobility to hot carrier effects. Although probable, such an assertion cannot be conclusively made for the $\mathrm{Ge}: \mathrm{Be}$ results at the present time.

The mobility of holes in the photoexcitation dominated region may provide information regarding the effect (if any) of $\mathrm{Be}^{+}$formation on carrier transport. First, the mobility of carriers under zero stress and 0.3 kbar is weakly dependent on temperature, but at $6.3 \mathrm{kbar}$ the mobility is constant. Temperature independent mobilities have also been observed at a stress of $1.7 \mathrm{kbar}$. Second, the mobility decreases slightly with the increase in photon flux for the zero stress and $0.3 \mathrm{kbar}$ but decreases slightly for $6.3 \mathrm{kbar}$. These observations present the following possibility: at zero stress and $0.3 \mathrm{kbar}$ the mobility decreases (with respect to both photon flux and temperature) due to the scattering contribution of $\mathrm{Be}^{+}$; for higher stresses at which no $\mathrm{Be}^{+}$can form, the mean energy of the holes increases with the increase in photon flux resulting in higher mobilities that are 
temperature independent for the same reason described for the Ge:Ga system.

In addition to showing that high compressive stress increases the mobility of holes in Al-doped Ge by a factor of 2, J.J. Hall ${ }^{9}$ showed that the mobility changes very little and may even go down in the limit of small applied compressions ( $<1 \mathrm{kbar}$ ). Figure 4.12 not only shows that the mobility of holes increases by a factor of 4 in the high stress limit (chosing the mobility maxima located at $17 \mathrm{~K}$ for comparison) but also reveals an increase of a factor of two for low stresses ( $0.3 \mathrm{kbar}$ for example). One may argue that Hall measurements taken at $6.2 \mathrm{~K}$ should not be compared to those at $17 \mathrm{~K}$ (due to differences in the Hall factor and in the contribution of the various scattering mechanisms), yet Figure 4.13 indicates strongly that $0.3 \mathrm{kbar}$ of stress will result in a significant increase even at lower temperatures. The reason for this is not well understood at the present time.

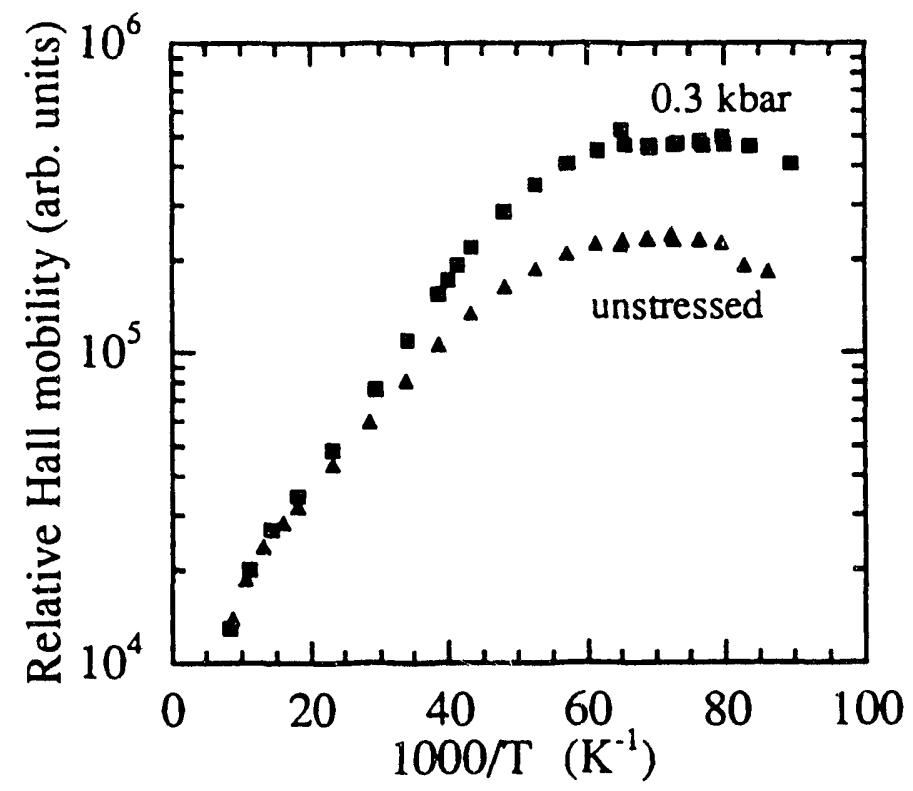

Figure 4.13. Relative Hall mobilities for holes in sample 728-8.7\#3. The data were taken in the dark. 


\subsection{Recombination coefficients for the $\mathrm{Ge}: \mathrm{Be}$ and $\mathrm{Ge}: \mathrm{Ga}$ systems}

The carrier lifetime has been previously defined as the inverse of the product of the trap concentration and the recombination coefficient which is in itself the product of the mean carrier velocity and the capture cross section. Figure 4.14 is a plot of the product of the recombination coefficient and the degeneracy factor. For the sake of simplicity these will be lumped into a single term, the effective recombination coefficient (ERC). In principle, the ERC for shallow hydrogenic impurities should be very similar in a particular host material such as $\mathrm{Ge}$ or $\mathrm{Si}$ since the mean velocity is specific to the host and the capture process is thought to be determined at highly delocalized, bound excited states (in which case the effect of the impurity core is reduced for p-like states). Thus, a plot of the ERC versus temperature reveals the degree of similarity among the various capture centers. The ERC's in Figure 4.14 were obtained by dividing the calculated lifetimes by the trap concentrations (assumed to be equal to the minority impurity concentration in every case). In addition, recombination coeffiecients were extracted for comparison from the data of Kaufman et al. 53 for $\mathrm{Ge}: \mathrm{Al}\left(5.7 \times 10^{12} \mathrm{~cm}^{-3} \mathrm{Al}, 2.7 \times 10^{12} \mathrm{~cm}^{-3}\right.$ donors) and $\mathrm{Ge}: \mathrm{Sb}(\mathrm{n}-$ type, $4.5 \times 10^{13} \mathrm{~cm}^{-3} \mathrm{Sb}, 5 \times 10^{12} \mathrm{~cm}^{-3}$ acceptors).

The values obtained in this study have been calculated based on the assumption of having a perfect blackbody and unity quantum efficiency (Section 3.2.2). In essence the values provide an upper limit to the magnitudes of the ERC's. However, the temperature dependence and the relative changes for a given sample with respect to the applied stress should in general be correct, for the determination of the carrier concentration has been done independent of these optical considerations which are some 
of the most challenging aspects of this experiment. The quantification of optical ionization cross sections would be helpful. Experimental reproduciblity was satisfactory. This is best exemplified by the fact that the zero stress ERC's for 773-6.8 and 783-4.4 are very similar even though they are from different crystals of slightly different dopant concentrations (for both majority and more importantly minority impurities).

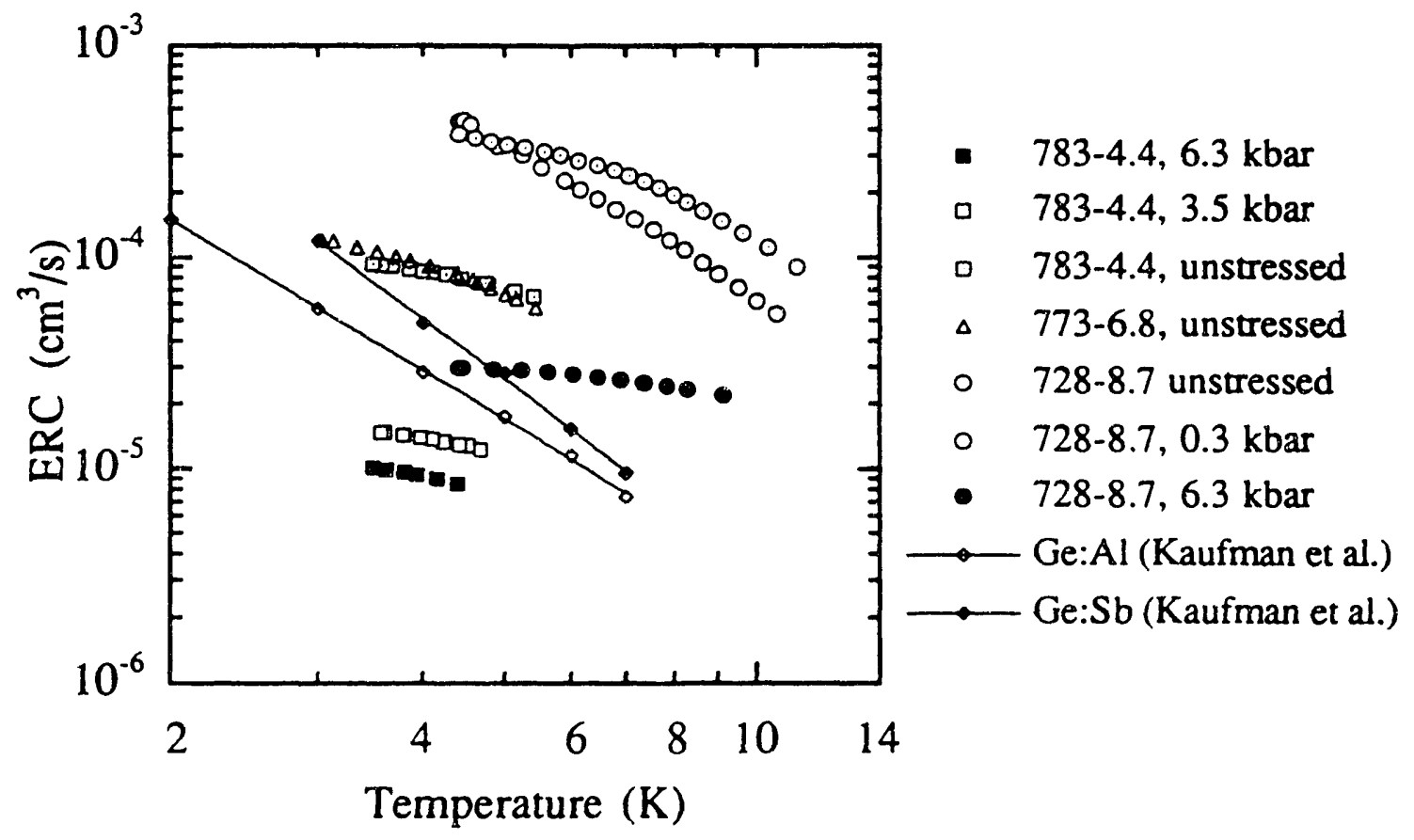

Figure 4.14. Effective recombination coefficients (ERC's) as a function of temperature for the materials and states of stress listed next to the legends.

As mentioned in Sec. 4.2, the weakness of the temperature dependence of $\mathrm{Ge}: \mathrm{Ga}$ data suggests the existence of hot holes. The factor of ten increase between the stressed and unstressea states may be due to the effective mass dependence of the ERC's. If the holes were in thermal 
equilibrium with the lattice, one could examine if the decrease in the ERC with stress at a particular temperature corresponds directly with that expected from changes in the degeneracy factor, the mean hole velocities, and the capture cross sections (as modeled by Lax or Abakumov et al.) due to their dependence on the hole effective mass.

The lifetime associated with hot carriers has in the past ${ }^{59}$ been related to the lifetime under thermal equilibrium, $\tau_{0}$, by the following equation:

$$
\tau_{\text {hot }}=\tau_{0}\left(\frac{\varepsilon}{3 \mathrm{k}_{\mathrm{B}} \mathrm{T} / 2}\right)^{1 / 2}
$$

where $\varepsilon$ is the energy associated with a hot carrier. Thus, to obtain the measured lifetime one must average over the distribution of carrier energies. If one approximates this distribution by that of the photons absorbed by the sample (meaning that the lifetime is equal to the energy relaxation time), one immediately sees that $\tau_{\text {hot }}$ is proportional to $\tau_{0} / T^{1 / 2}$. That is, the measured recombination coefficient is proportional to the product of the "true" coefficient and the square root of temperature. In the Ge:Ga system the measured ERC's produce a "true" coefficient proportional to $T^{-n}$ where $n=1.5 \pm 0.3$ compared to $n=2.36(\mathrm{Ge}: \mathrm{Al})$ and 2.94 . (Ge:Sb) for the results of Kaufman et al. ${ }^{54}$ In addition, the assumption of a carrier energy distribution similar to that of the absorbed photons leads to another important simplification: at a given temperature the increase with stress of the lifetime of the hot carriers should be the same as the lifetime for carriers having a thermal distribution.

The increase of the free hole lifetime due to the application of a large uniaxial stress can be estimated. As indicated in Chapter 2, 


$$
\tau_{0}=\frac{1}{\beta N_{t} v_{t h} \sigma}
$$

where $\beta$ is the valence band degeneracy factor, $N_{t}$ is the trap concentration, $v_{\text {th }}$ is the hole thermal velocity, and $\sigma$ is the capture cross section. For large applied stresses $\beta$ has a value of 2 rather than 4 . The effect of stress on the velocity and the capture cross section appears via their functional dependence on the hole effective mass. The thermal velocity is proportional to $\left(m^{*}\right)^{-1 / 2}$ while $\sigma$ is proportional to $\left(m^{*}\right)^{3}$ (given by Eq. 2.22a). Thus, $\tau_{0}$ is proportional to $\beta^{-1}\left(m^{*}\right)^{-5 / 2}$, and by Eq. 2.6 the effective mass is proportional to the acceptor binding energy $E$. The increase in the lifetime of $\mathrm{Ge}: \mathrm{Ga}$ for a stress of $6.3 \mathrm{kbar}$ applied in a $<100>$ direction is

$$
\frac{\left(\tau_{0}\right)_{\text {stressed }}}{\left(\tau_{0}\right)_{\text {unstressed }}}=\frac{\left(\beta \mathrm{m}^{*}\right)_{\text {unstressed }}}{\left(\beta \mathrm{m}^{5 / 2}\right)_{\text {stressed }}}=\frac{\left(\beta \mathrm{E}^{5 / 2}\right)_{\text {unstressed }}}{\left(\beta \mathrm{E}^{5 / 2}\right)_{\text {stressed }}}=\frac{4(11.07)^{5 / 2}}{2(6.7)^{5 / 2}}=7 .
$$

This is in fair agreement with the factor of ten increase observed for sample 783-4.4 (Fig. 4.5 and Table 4.3).

Finally, the Ge:Be system presents a particularly interesting situation. Under large stresses the ERC does not vary very much with temperature, yet in the unstressed case the temperature dependence is similar to that of the data of Kaufman et al. for Ge:Al. In other words, the mobility data for unstressed $\mathrm{Ge}: \mathrm{Be}$ has the signature of hot carriers (Sec. 4.3.2), but the concentration of photoexcited holes-which is inversely proportional to the ERC for a constant trap concentration-decreases with temperature in a manner observed for thermalized carriers. Any explanation of this result is purely speculative at this point. Barring major experimental errors, the temperature dependence possibly results from the 
changes in the trap concentration and not the ERC. Thus, for complex systems like $\mathrm{Ge}: \mathrm{Be}$ one cannot obtain the effective recombination coefficient by simply multiplying the lifetime by the minority impurity concentration as was done for Figure 4.14. 


\section{Conclusion}

The photo-Hall effect of uniaxially stressed, p-type Ge has been successfully measured. The application of large stresses to $\{100\}$-oriented sample surfaces produces a one order of magnitude increase in the concentration of photoexcited holes in both Ge:Ga and Ge:Be crystals. These results suggest that the hole lifetime also increases significantly with stress. The origin of this effect is not yet well understood but may be associated with the effect of stress on the hole effective mass. In addition, the Hall mobilities exhibit an increase of a factor of two in Ga-doped Ge and a factor of four in Be-doped Ge. The enhancement of the Hall mobilities is due to stress-induced changes of the conductivity effective mass of holes.

There are two major differences between the photo-Hall data of $\mathrm{Ge}: \mathrm{Ga}$ and Ge:Be. First, the temperature dependence of the optically generated holes is very sensitive to the application of stress in Be-doped Ge but not sensitive in Ga-doped Ge. Second, the stress-induced increase of the Hall mobility in $\mathrm{Ge}: \mathrm{Be}$ is two times greater than that observed in Ge:Ga. These two effects suggest the following: the stress-induced increases in the hole concentration and Hall mobility do not depend on whether the dopant is $\mathrm{Ga}$ or $\mathrm{Be}$; however, the details of the hole capturing and scattering processes are determined by the nature of the dopant. In other words, stress produces certain changes in the Ge. The changes in (a) the host material (including the deformatior of the Ge band structure) and (b) general features of the effective mass theory lead to the major effects observed. Shallow single acceptors will probably react to stress in a 
similar manner. The degree of similarity of multilevel acceptors such as $\mathrm{Cu}, \mathrm{Zn}$, and $\mathrm{Be}$ at low temperatures is not clear.

Finally, the photo-Hall data can provide information regarding stress related changes of the photocurrent in Ge photoconductors. The results establish a fundamental limit as to how much the photocurrent can change with stress when a constant number of holes is being photogenerated. This limit is given by the product of the factors by which the lifetime and mobility increase (approximately equal to 20 for the Ge:Ga system and the experimental conditions described in this thesis). Any deviation from this limit is due to the effect of stress on other aspects of the detector including the impurity absorption coefficient and the electrical contacts. By understanding the behavior of fundamental physical properties such as lifetime and mobility, one will be able to determine if the performance of a photoconductor can be further improved. 


\section{References}

1. Bratt, P.R., Impurity Germanium and Silicon Infrared Detectors, in Semiconductors and Semimetals, R.K. Willardson and A.C. Beer, Editor. Vol. 12. 1977, Academic Press: New York. p. 39.

2. Eisenman, W.L., J.D. Merriam, and R.F. Potter, Operational Characteristics of Infrared Photodetectors, in Semiconductors and Semimetals, R.K. Willardson and A.C. Beer, Editor. Vol. 12. 1977, Academic Press: New York. p. 1.

3. Shenker, H., W.J. Moore, and E.M. Swiggard, J. App. Phys., 1964. 35: p. 2965.

4. Burnstein, E., et al., Phys. Rev., 1954. 93: p. 65.

5. Kaiser, W. and H.Y. Fan, Phys. Rev., 1954. 93: p. 977.

6. Moore, W.J. and H. Shenker, Infrared Phys., 1965. 5(99).

7. Haller, E.E., M.R. Hueschen, and P.L. Richards, Appl. Phys. Lett., 1979. 34: p. 495.

8. Koenig, S.H. and J.J. Hall, Phys. Rev. Lett., 1960. 5: p. 550.

9. Hall, J.J., Phys. Rev., 1962. 128: p. 68.

10. Hummel, R.E., Electronic Properties of Materials. 1985, New York: Springer-Verlag. p.88.

11. Chelikowsky, J.R. and M.L. Cohen, Phys. Rev. B, 1976. 30: p. 556.

12. Wachs, A.L., et al., Phys. Rev. B, 1985. 32: p. 2326.

13. Smith, R.A., Semiconductors. 2nd ed. 1978, Cambridge: Cambridge University Press.

14. Dresselhaus, G., A.F. Kip, and C. Kittel, 1955. 98: p. 368.

15. Dexter, R.N., H.J. Zeiger, and B. Lax, Phys. Rev., 1956. 104: p. 637. 
16. Haller, E.E. and W.L. Hansen, Solid State Comm., 1974. 15: p. 687.

17. Shenker, H., E.M. Swiggard, and W.J. Moore, Transactions of the Metallurgical Society of AIME, 1967. 239: p. 347.

18. Haller, E.E., et al., Phys. Rev. Lett., 1983. 51: p. 1089.

19. Blakemore, J.S., Semiconductor Statistics. 1987, New York: Dover.

20. McKelvey, J.P., Solid State and Semiconductor Physics. 1966, New York: Harper and Row. 308.

21. Erginsoy, C., Phys. Rev., 1950. 79: p. 1013.

22. Rutherford, E., Phil. Mag., 1911. 21: p. 669.

23. Pierret, R.F., Advanced Semiconductor Fundamentals. Modular Series on Solid State Devices, ed. R.F. Pierret and G.W. Neudeck. Vol. VI. 1987, Reading, Massachusetts: Addison-Wesley Publishing Company.

24. Thomson, J.J., Phil. Mag., 1924. 47: p. 337.

25. Lax, M., Phys. Rev., 1960. 119: p. 1502.

26. Gerasimov, A.B., et al., phys. stat. sol. (b), 1987. i44: p. K99.

27. Khan, F.A. and D.P. Bhattacharya, Solid State Comm., 1984. 51: p. 719.

28. Abakumov, V.N. and I.N. Yassievich, Sov. Phys. JETP, 1976. 44: p. 345.

29. Abakumov, V.N., V.I. Perel', and I.N. Yasslevich, Sov. Phys.Semicond., 1978. 12: p. 1.

30. Ascarelli, G. and S. Rodriguez, J. Phys. Chem. Solids, 1961. 22: p. 57.

31. Ascarelli, G. and S. Rodriguez, Phys. Rev., 1961. 124: p. 1321.

32. Brown, R.A. and S. Rodriguez, Phys. Rev., 1967. 153: p. 890. 
33. Darken, L.S. and G.E. Jellison Jr., Appl. Phys. Lett., 1989. 55: p. 1424.

34. Bemski, G., Proc. Inst. Radio Engrs., 1958. 46: p. 990.

35. Germer, T.A., N.M. Haegel, and E.E. Haller, J. Appl. Phys., 1986. 60: p. 1055.

36. Hall, E.H., Amer. J. Maths., 1879. 2: p. 287.

37. Putley, E.H., The Hall Effect and Related Phenomena. 1960, London: Butterworths.

38. Beer, A.C. and R.K. Willardson, Phys. Rev., 1958. 110: p. 1286.

39. Pikus, G.E. and G.L. Bir, Sov. Phys.-Solid State, 1959. 1: p. 1502.

40. Bir, G.L. and G.E. Pikus, Symmetry and Strain-Induced Effects in Semiconductors. New York: John Wiley \& Sons.

41. Buczko, R. and J.A. Chroboczek, Phil. Mag. B, 1984. 50: p. 429.

42. Dickey, D.H. and J.O. Dimmok, J. Phys. Chem. Solids, 1967. 28: p. 529.

43. Kazanskii, A.G., P.L. Richards, and E.E Haller, Solid State Comm., 1977. (24): p. 603.

44. Price, P.J., Phys. Rev., 1961. 124: p. 713.

45. Broeckx, J. and J. Vennik, Phys. Rev. B, 1987. 12: ß. 6165.

46. Smith, C.S., Phys. Rev., 1954. 94: p. 42.

47. Hasegawa, H., Phys. Rev., 1963. 129: p. 1029.

48. Hensel, J.C. and G. Feher, Phys. Rev., 1963. 129: p. 1041.

49. Hensel, J.C., Solid State Comm., 1966. 4: p. 231.

50. van der Pauw, L.J., Philips Res. Rep., 1958. 13: p. 1. 
51. Wang, J.-Q., P.L. Richards, J.W. Beeman, and E.E. Haller, Appl. Opt., 1987. 26: p. 4767.

52. Weinstock, H. and J. Parpia, A survey of thermometric characteristics of recently produced Allen-Bradley/Ohmite resistors, in Temperature, Its Measurement and Control in Science and Industry, H.H. Plumb, Editor. 1971, Instrument Society of America: Pittsburgh. p. 785.

53. Lamb, W.E., Jr., Phys. Rev., 1946. 70: p. 308.

54. Kaufman, S.A., K.M. Kulikov, and N.P. Likhtman, Soviet Phys.Semicond., 1970. 4: p. 102.

55. Sclar, N. and E. Burstein, Phys. Rev., 1955. 98: p. 1757.

56. Koenig, S.H., R.D. Brown III, and W. Schillinger, Phys. Rev., 1962. 128: p. 1668.

57. Ohmura, Y., Solid State Comm., 1991. 79: p. 1029.

58. Haegel, N.M., et al., Phys. Rev. B, 1989. 39: p. 3677.

59. Norton, P. and H. Levinstein, Phys. Rev. B, 1972. 6: p. 478.

60. Noguera B., A. and C.J. Hearn, Solid-State Electronics, 1978. 21: p. 171. 


\section{Appendix A}

The amount of uniaxial stress applied by the stressing coldfinger (Fig. 3.4) on a bar-shaped sample can be calculated from a simple structural model. The leaf spring is treated as a beam overhanging one support with a concentrated load at the end of the overhang (Figure A.1). A force $P$ deflects the leaf spring by an amount $\Delta c$. This is achieved by turning the \#4-40 screw as shown in Fig. A.1. This force produces a force at point $B$ that is given by

$$
\mathrm{R}=\frac{\mathrm{P}}{l}(l+\mathrm{a})
$$

where $l$ and a are fixed by the coldifinger design. The force $\mathrm{P}$ is expressed in terms of $\Delta c, a, E$, and I by the following equation:

$$
\mathrm{P}=\frac{3 \mathrm{I} \Delta \mathrm{cE}}{(l+\mathrm{a}) \mathrm{a}^{2}} .
$$

$\mathrm{E}$ is the Young's modulus and I is the moment of inertia of the spring leaf. The amount of uniaxial stress applied to the bar sample is simply $\mathrm{R}$ divided by the cross-sectional area of the bar.

The characteristics of the leaf spring determine $P$ in two ways. First, the Young's modulus $\mathrm{E}$ is that of the spring material. Second, the dimensions of the spring determine the magnitude of I. The moment of inertia $I$ is equal to $w t^{3} / 12$, where $w$ is the width and $t$ is the thickness of the leaf spring. Because the moment of inertia is proportional to the cube of the spring thickness, $\mathrm{P}$ is also proportional to $\mathrm{t}^{3}$. Consequently, the 


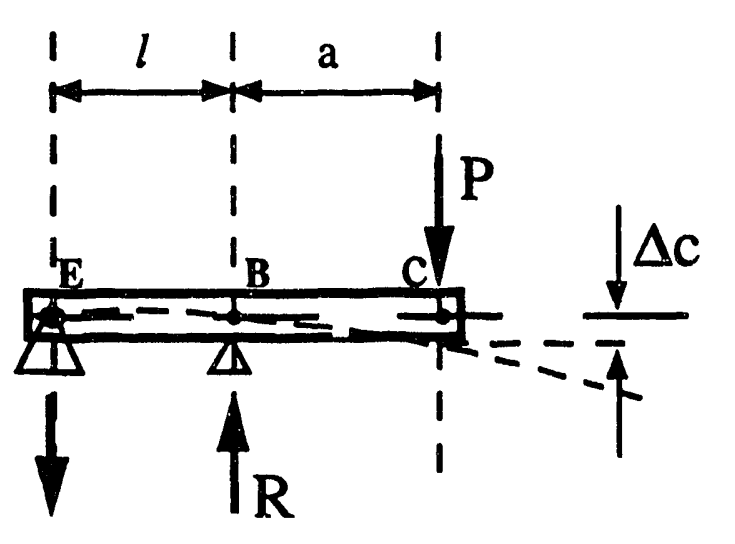

Structural model
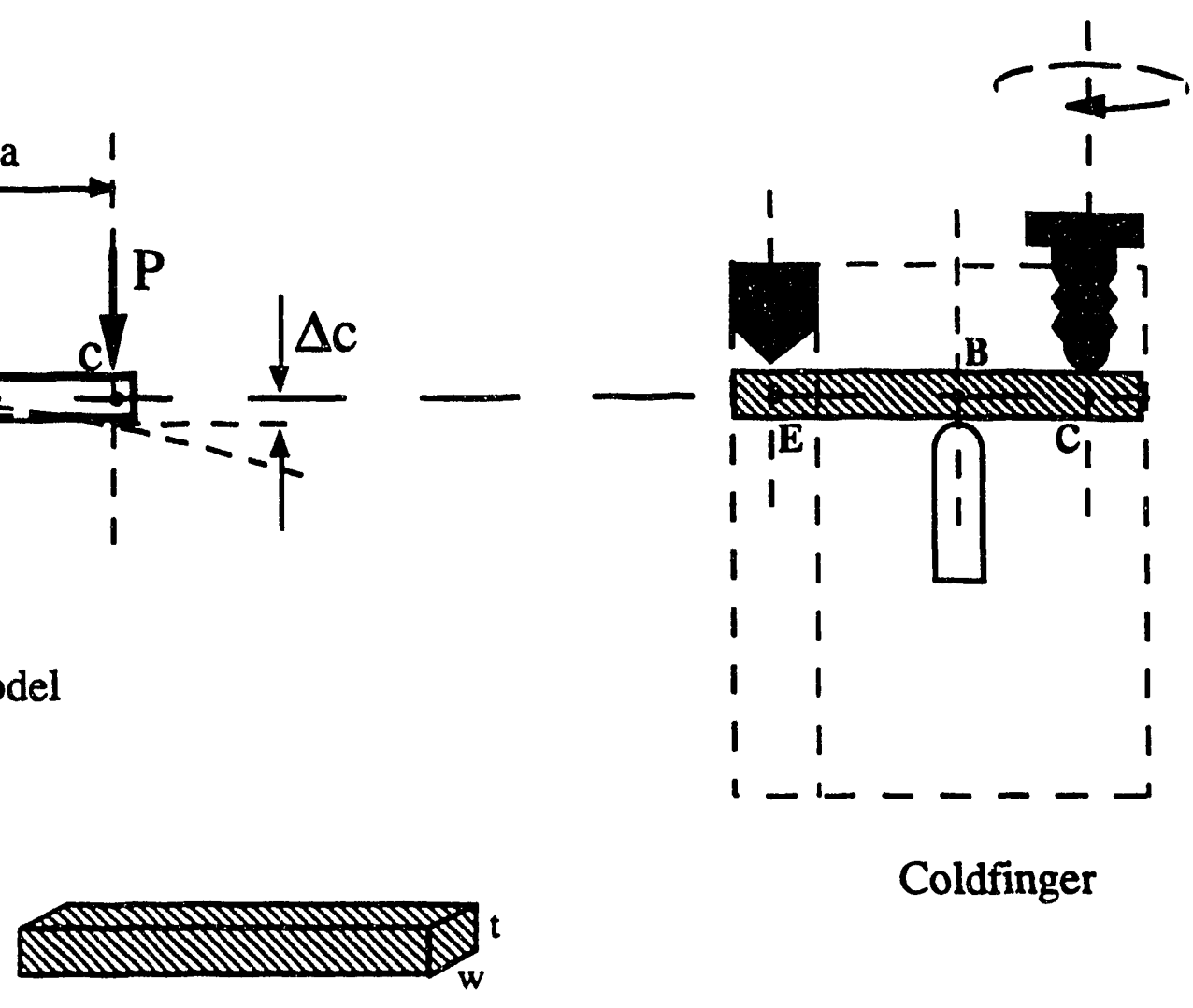

Leaf spring

Coldfinger

Figure A.1.

amount stress applied to the bar sample is strongly dependent on the thickness of the leaf spring.

Leaf springs can be stacked in order to obtain large uniaxial stresses. The total force P' necessary to deflect the stack of springs by an amount $\Delta c$ is equal to the sum of the forces required to deflect each spring by an amount $\Delta c$. It is possible to achieve large stresses by using a thicker spring. There are two important factors which must be weighed when choosing the appropriate configuration. First, the deflection of the leaf spring(s) should be sufficient to achieve a stable applied stress as the 
coldfinger is cooled to $4 \mathrm{~K}$. Second the leaf-spring configuration should not be deflected beyond its elastic deformation limit; that is, plastic deformation of the spring(s) should be avoided.

The stress measurements performed in this study had several aspects in common. All springs were made of phosphor bronze ( $\left.E=1.1 \times 10^{3} \mathrm{kbar}\right)$. Second, the coldfinger was designed such that the dimensions $l$ and " $\mathrm{a}$ " are equal to each other. Third, the cross sections of all samples were $1 \times 1$ $\mathrm{mm}^{2}$. Finally, the \#4-40 stressing screw was tumed about $1 / 4$ of a revolution. The screw label \#4-40 refers to a \#4 screw with 40 threads per inch. Therefore, $1 / 4$ of a revolution produced a $\Delta c$ of 0.00625 " or 0.016 $\mathrm{cm}$. The spring-leaf configurations that were used are described in Table A.1. The error in the estimated stresses is of the order of $10 \%$.

\begin{tabular}{|c|c|c|c|}
\hline $\begin{array}{c}\text { Spring thickness, } \\
\mathrm{t}(\mathrm{cm})\end{array}$ & $\begin{array}{c}\text { Spring width, } \\
\mathrm{w}(\mathrm{cm})\end{array}$ & No. of springs & $\begin{array}{c}\text { Estimated stress } \\
\text { (kbar) }\end{array}$ \\
\hline 0.84 & 0.47 & 1 & 0.3 \\
\hline 0.16 & 0.47 & 1 & 1.7 \\
\hline 0.16 & 0.47 & 2 & 3.5 \\
\hline 0.25 & 0.46 & 1 & 6.3 \\
\hline
\end{tabular}

Table A.1.

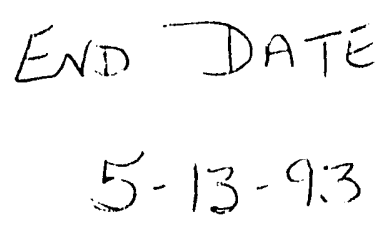

